\title{
Pickup ions at Dione and Enceladus: Cassini Plasma Spectrometer simulations
}

\author{
Ed C. Sittler, ${ }^{1}$ R. E. Johnson, ${ }^{2}$ S. Jurac, ${ }^{3}$ J. D. Richardson, ${ }^{3}$ M. McGrath, ${ }^{4}$ F. Crary, ${ }^{5}$ \\ D. T. Young, ${ }^{5}$ and J. E. Nordholt ${ }^{6}$ \\ Received 15 August 2002; revised 15 April 2003; accepted 18 July 2003; published 20 January 2004.
}

[1] Voyager images of the icy satellites of Saturn, Dione and Enceladus, suggest that they may have been geologically active and are not only composed of ice. Recent observations by the Hubble Space Telescope have shown the presence of ozone at both Dione and Rhea, which also implies the presence of molecular oxygen at these bodies. Observations of Ariel, Europa, Ganymede, and Callisto indicate the presence of $\mathrm{CO}_{2}$, so its presence on the Saturnian satellites is also expected. The Cassini Plasma Spectrometer (CAPS) will provide the capability to determine the global composition of these bodies by measuring the pickup ions produced by the ionization of their sputter-produced atmospheres. We will present a model of these atmospheres and associated pickup ions and demonstrate CAPS ability to distinguish the freshly produced picked up ions from the ambient plasma. Such ions are expected to form a ring distribution that will have a uniquely different energy-angle dependence than the ambient plasma ions. In the case of Dione we expect the potential for a moderate strength interaction for which both Voyager 1 and Pioneer 11 spacecraft measured ion cyclotron waves centered on the Dione L shell and near the equatorial plane. SKR radio emissions also displayed emissions occurring at the orbital period of Dione which could indicate some intrinsic activity due to Dione. So again, something interesting may be going on at Dione. Since Enceladus, or material in orbit near Enceladus, may be the source of the E-ring, some surprises may be encountered during its close encounter with the Cassini spacecraft. In the case of Dione we will show that a wake pass at $500 \mathrm{~km}$ altitude is more than an order of magnitude better than an upstream pass at $500 \mathrm{~km}$ altitude. Pickup ion detection for minor ion species such as $\mathrm{NH}_{3}^{+}$ is possible for $500 \mathrm{~km}$ altitude wake pass but not for $\approx 500 \mathrm{~km}$ altitude upstream pass at closest approach. For navigation reasons a $100 \mathrm{~km}$ pass is not allowed. Therefore it is essential to have a wake pass to maximize the science return for a targeted flyby with Dione. The CAPS observations when combined with magnetometer, plasma wave and energetic particle observations will allow us to estimate the source of ions into Saturn's magnetosphere due to these two bodies and to characterize the nature of the interaction with Saturn's magnetosphere. INDEX TERMS: 6280 Planetology: Solar System Objects: Saturnian satellites; 6055 Planetology: Comets and Small Bodies: Surfaces and interiors; 6005 Planetology: Comets and Small Bodies: Atmospheres-composition and chemistry; 7807 Space Plasma Physics: Charged particle motion and acceleration; KEYWORDS: icy satellites, pickup ions, Dione,

Enceladus, composition

Citation: Sittler, E. C., R. E. Johnson, S. Jurac, J. D. Richardson, M. McGrath, F. Crary, D. T. Young, and J. E. Nordholt (2004), Pickup ions at Dione and Enceladus: Cassini Plasma Spectrometer simulations, J. Geophys. Res., 109, A01214, doi:10.1029/2002JA009647.

\footnotetext{
${ }^{1}$ NASA Goddard Space Flight Center, Greenbelt, Maryland, USA.

${ }^{2}$ University of Virginia, Charlottesville, Virginia, USA. USA.

${ }^{3}$ Massachusetts Institute of Technology, Cambridge, Massachusetts,

${ }^{4}$ Space Telescope Science Institute, Baltimore, Maryland, USA.

${ }^{5}$ University of Michigan, Ann Arbor, Michigan, USA.

${ }^{6}$ Los Alamos National Laboratory, Los Alamos, New Mexico, USA.

Copyright 2004 by the American Geophysical Union. 0148-0227/04/2002JA009647
}

\section{Introduction}

[2] In this paper we will be primarily concerned with the interaction of Saturn's magnetosphere with its icy satellites Dione and Enceladus. In the future we plan to also look at the other icy satellites Mimas, Tethys, and Rhea; in the case of Rhea, finite gyroradii effects will be important. The icy satellite interaction with Saturn's magnetosphere can provide very important information about the bulk properties and surface properties of these bodies by detecting the 
pickup ions produced by the ionization of their sputtered neutral atmosphere [see Johnson and Sittler, 1990]. The icy satellites are believed to be composed primarily of ice with trace amounts of $\mathrm{O}_{2}, \mathrm{O}_{3}$, and other possible frozen volatiles such as $\mathrm{CH}_{4}, \mathrm{NH}_{3}, \mathrm{CO}_{2}$, and possible dark components such as C and S [see Morrison et al., 1984].

[3] At present, most of our knowledge of the Saturnian system can be traced to the pioneering observations of Saturn's plasma population, energetic particle population, magnetic fields, plasma waves, and remote sensing observations from the Pioneer 11 and Voyager 1 and 2 encounters. The papers by Frank et al. [1980], Lazarus and McNutt [1983], Sittler et al. [1983], and Richardson [1986] summarize the first observations of Saturn's plasma environment. The energetic particle populations of Saturn's magnetosphere were summarized in papers by Fillius and McIlwain [1980], Van Allen et al. [1980a, 1980b], Van Allen [1982], Fillius et al. [1980], Vogt et al. [1981, 1982], Krimigis and Armstrong [1982], Schardt and McDonald [1983], Schardt et al. [1984], Krimigis et al. [1983], and Van Allen [1984]. More recently, Maurice et al. [1996], Paranicas et al. [1997] and Jurac et al. [2001a] have reanalyzed the Voyager plasma and energetic particle data to put it in a more synergistic form. Richardson and Sittler [1990] combined the Voyager ion and electron plasma observations to construct an empirical model of Saturn's plasma environment inside of about $15 \mathrm{R}_{\mathrm{S}}$. In concert, Johnson et al. [1989] constructed from all these observations a model of a neutral cloud torus that surrounds Saturn and is primarily due to sputtered neutrals coming from the icy satellites of Saturn. Richardson et al. [1986] made the first attempt at combining all these various data sets to construct a semiempirical model of Saturn's plasma and neutral environment including various collisional and ionization processes and radial diffusion which enforced constraints on the plasma source strength and the rate of radial diffusion.

[4] This work was followed by the discovery using HST of a relatively large toroidal cloud of $\mathrm{OH}$ that was surrounding Saturn with densities as high as $500 \mathrm{~cm}^{-3}$ near the L shell of Enceladus [Shemansky et al., 1993; Hall et al., 1996; Richardson et al., 1998]. This density was more than an order of magnitude larger than that predicted by Johnson et al. [1989] in which the icy satellites were the principal source of neutrals. In order to reconcile these neutral density observations with the plasma density observations, Richardson et al. [1998] had to invoke rapid radial transport with time scales at $6 R_{S}$ of only 5 days while in the original work by Richardson et al. [1986] it was 700 days. Using measurements of sputtering yields and available plasma data Jurac et al. [2001b] showed that the icy satellites and the observed grains in the $\mathrm{E}$ ring each contribute less than $10 \%$ of the observed neutral population. Other possible sources have been examined, including the possibilities that more material is present near Enceladus and the surface materials are more volatile [Jurac et al., 2002] or there is a selfsustained interaction between Enceladus and the E-ring [Hamilton and Burns, 1994].

[5] The detection of ozone, $\mathrm{O}_{3}$, in the surface ice of Rhea and Dione [Noll et al., 1997] for which the column densities were $\sim 2 \times 10^{16} \mathrm{~mol} / \mathrm{cm}^{2}$ meant that column densities of $\mathrm{O}_{2}$ could be as high a $10^{19} \mathrm{~mol} / \mathrm{cm}^{2}$ within the icy mantles of these bodies. Since the temperature of these bodies are $\sim 75^{\circ} \mathrm{K}$ to $100^{\circ} \mathrm{K}$ [Hanel et al., 1981, 1982], there could exist a significant gravitationally bound $\mathrm{O}_{2}$ atmosphere at Dione and Enceladus with the scale heights for $\mathrm{O}_{2}$ of $117 \mathrm{~km}$ and $227 \mathrm{~km}$, respectively. If the $\mathrm{O}_{2}$, believed to be trapped in the icy surfaces of these bodies, contributes to these atmospheres by diffusion from depth, then, based on the formation rate, the peak neutral densities could be as high as $8.3 \times 10^{11} \mathrm{~mol} / \mathrm{cm}^{3}$ for Dione and $4.3 \times 10^{11} \mathrm{~mol} / \mathrm{cm}^{3}$ for Enceladus [see Spencer, 1998]. Therefore a wake pass of Dione may see large quantities of pickup $\mathrm{O}_{2}^{+}$and $\mathrm{O}_{3}^{+}$near the limb of Dione; while an upstream pass at $500 \mathrm{~km}$ altitude would probably not detect such an atmosphere. In the case of Enceladus the atmosphere would be less bound with a significant fraction of the $\mathrm{O}_{2}$ and $\mathrm{O}_{3}$ on escape trajectories. We may also expect significant amounts of $\mathrm{CO}_{2}$ to be present, since $\mathrm{CO}_{2}$ was detected at the Galilean moons [McCord et al., 1998], in the surface of Ariel [Grundy et al., 2002], and in the atmosphere of Titan [Samuelson et al., 1983] where CO was also detected [ Lutz et al., 1983]. The planned close encounters by Cassini of these bodies will allow us to develop a more accurate picture of their role in neutral cloud production and thus in the plasma and energetic particle production within Saturn's magnetosphere.

[6] Since these bodies are deep within the magnetosphere of Saturn and its radiation belts, they are expected to have, at minimum, a tenuous atmosphere due to sputtering induced by energetic particle bombardment and bombardment by the ambient plasma. The icy satellites should also be exposed to micrometeorite bombardment, which will produce a porous regolith on their surface and the emission of particulates into Saturn's magnetosphere [see Ip, 1997].

[7] Knowledge about the composition of their atmospheres can also provide information about the surface composition [Johnson and Sittler, 1990]. In the case of Dione the leading hemisphere is brighter than the trailing hemisphere [Morrison et al., 1984]. This suggests, as is the case at Europa [Johnson et al., 2004a], that darkening by plasma and energetic particle bombardment is competing with meteoroid mixing of the surface. There is also evidence of a global tectonic system of fractures and faults for Dione [Morrison et al., 1984]. In the case of Enceladus fractured and smooth plains dominate which indicates its surface is relatively young $\sim 10^{8}$ years [Morrison et al., 1984]. Enceladus is also associated with Saturn's E ring whose brightness peaks at Enceladus' L shell [Baum et al., 1980, 1981]. The evidence for tectonic activity for both bodies could indicate the presence of, for example, a water-ammonia eutectic which melts at $170^{\circ} \mathrm{K}$ within the interior of these bodies [Morrison et al., 1984]. The close encounters planned for these bodies by Cassini will provide accurate measurements of the plasma and energetic particle populations at these bodies. This data, when combined with the latest laboratory measurements of the sputtering processes [Johnson, 1990, 1998; Baragiola et al., 2003; Johnson et al., 2003b], can provide accurate models of their sputtered atmospheres at the time of the encounters.

[8] Since the sputtering yields of the icy surfaces of the Saturnian satellites are large $\left(\sim 50\right.$ to $1000 \mathrm{H}_{2} \mathrm{O}$ mol/ion [Johnson, 1990]), trace refractory species are expected to be carried off with the water molecules. Sputtering does 
produce ions directly but with low efficiency relative to neutrals. The ions are accelerated immediately. Depending on the flow field, those produced on the trailing side will be driven back in and those on the leading side might be swept away and detected by CAPS for a wake pass. However, this issue is more complicated because these bodies can become charged which will effect ion production. This needs further study and will be considered in the future. Electron sputtering as described in reference does need to be considered in future work but is not considered here. Electrons do contribute to sputtering but inefficiently compared with heavy ions. The yields are comparable to those for protons at the same velocity. However, they can produce decomposition with $\mathrm{O}_{2}$ diffusing out, as at Europa [Johnson et al., 2004a, 2004b]. Although some information about the icy satellite composition can be acquired at large distances, the detected species will be partially equilibrated and dissociated, and therefore the origins will be more difficult to determine. Furthermore, when sampling the sputter-produced atmosphere of these bodies, the fresh pickup ions will form unique ring distributions. These can be readily distinguished from the ambient ions, which obey bi-Maxwellian distributions. Pickup ions formed from the ambient neutrals will also form ring distributions, but they will have accumulated over periods $\sim 10$ hour rotation period of Saturn and are expected to have pitch angle scattered to shell distributions or bi-Maxwellian distributions before being observed [see Vasyliunas and Siscoe, 1976]. See section 5 for a description of a ring distribution. A shell distribution, as described by Vasyliunas and Siscoe [1976], is a spherical shell in the plasma frame where the particles are concentrated in velocity space. Bi-Maxwellian distributions have an exponential dependence on energy and the temperature, $\mathrm{T}_{11}$, associated with motion along $\vec{B}$ is different from the temperature, $\mathrm{T}_{\perp}$, associated with motion perpendicular to $\vec{B}$.

[9] The atmospheres of these bodies can be sampled by their conversion to ions (photoionization, electron impact ionization, and charge exchange). These pickup ions can then be measured by the ion mass spectrometer (IMS) of the Cassini Plasma Spectrometer Experiment (CAPS) (see D. T. Young et al. (Cassini Plasma Spectrometer investigation, submitted to Space Science Review, 2003, hereinafter referred to as Young et al., submitted manuscript, 2003) for instrument description). The CAPS experiment will also provide measurements of the ambient plasma, ions, and electrons, which will be important for estimating the charge exchange and electron impact ionization rates for the pickup ions. The Magnetospheric Imaging Instrument (MIMI) (see Krimigis et al. [2004] for instrument description) will provide the hot plasma and energetic particle observations required to estimate the sputtering rates at the time of the targeted flybys. We note that the pickup ions with their unique energy-angle signature can provide the most direct information about the atmosphere composition.

[10] As discussed by Johnson and Sittler [1990], an extensively sputtered surface will display the following properties: (1) volatile species are highly depleted so trace species may be hard to detect using reflectance spectra. (2) Sputtering rates for extensively sputtered surfaces are determined by the bulk composition hence CAPS can be more sensitive than reflectance experiment. (3) Younger surfaces, such as that present at Enceladus, will have higher yields. CAPS, which provides a very sensitive measurement of the icy satellite's bulk atomic and molecular composition, will be complementary to the information provided by the Visible Infrared Mapping Spectrometer (VIMS) (see R. H. Brown et al. (The Cassini Visual and Infrared Mapping Spectrometer Investigation, submitted to Space Science Review, 2003) for description of instrument) on Cassini which will provide spatial maps of surface composition. The Composition Infrared Spectrometer (CIRS) experiment on Cassini (see V. G. Kunde et al. (Exploring the Saturn system in the thermal infrared, manuscript in preparation for Space Science Reviews, 2003) for instrument description) will provide temperature maps of the icy satellite surfaces which will be important for estimating sputtering rates which are temperature dependent [see Johnson, 1990; Bar-Nun et al., 1985].

[11] Here we note that Pioneer 11 and Voyager 1 observations show that there may be a strong interaction between Dione and Saturn's magnetosphere. This is also supported by the simulations presented in this paper. For example, Pioneer 11 [Smith and Tsurutani, 1983] and Voyager 1 [Barbosa, 1993] magnetometer observations showed the presence of ion cyclotron waves in the vicinity of Dione's L shell and the frequency of the waves are consistent with the dominant pickup ion being a heavy ion such as $\mathrm{H}_{2} \mathrm{O}^{+}, \mathrm{OH}^{+}$, or $\mathrm{O}^{+}$. These waves, which are only observed close to the equatorial plane, provide indirect evidence for the formation of pickup ions in the vicinity of Dione. Galileo results near the Jovian satellites [Russell and Huddleston, 2000; Russell et al., 2000] showed that peaks in the magnetometer spectra give information about the composition of the pickup ions being produced at a satellite body and will thus complement the CAPS observations. If the interaction is strong enough then the pickup process will also generate an ion beam instability [Ma et al., 1987] in the plasma. Such instabilities produce a spectrum of electric and magnetic waves which can be measured by the Radio and Plasma Wave Science (RPWS) instrument on Cassini (see D. Gurnett et al. (Radio and plasma wave science investigation for Cassini, submitted to Space Science Review, 2003) for instrument description). Kurth et al. [1981] and Desch and Kaiser [1981] reports seeing a 66 hour periodicity in the SKR radio emissions as observed by Voyager 1; the orbital period of Dione is 65.68 hours. These radio emissions could be due to a shadowing effect with the plasma sheet but could also be due to some intrinsic interaction between Dione and the magnetosphere. If the satellite has a conductive interior then there may also be a magnetic signature as was the case for Galileo at Europa and Callisto [see Kivelson et al., 2000; Zimmer et al., 2000]. Since the discovery of an internal magnetic field at the Jovian satellite Ganymede [Kivelson et al., 1998], we cannot rule out the presence of an internal magnetic field, which would complicate the interaction and infer the presence of a dynamo operating in the core of the icy satellite. Although we think this unlikely, Cassini would be able to measure the presence of such a field if the flybys were close enough to the satellite. Finally, we note that the satellites are not expected to have significant conductive ionospheres and that the atmospheres are collisionally thin and will not be able to prevent the flowing 
plasma from reaching the satellite's surface. We emphasize that ionospheres are unlikely because the atmospheres are collisionally thin so that convective electric field is not attenuated significantly and that any ions formed are rapidly convected away. The importance of these observations during targeted flybys of these various bodies by Cassini emphasizes the need for wake encounters as close as possible to the icy satellite. This will maximize the science return from these targeted flybys, which will become clearer in later discussions.

\section{CAPS Instrument Description and Capabilities With Respect to Targeted Icy Satellite Encounters}

[12] The overall capabilities of the CAPS instrumentation are described in detail by Young et al. (submitted manuscript, 2003). The CAPS instrument is composed of an Ion Mass Spectrometer (IMS), Electron Spectrometer (ELS), and an Ion Beam Spectrometer (IBS). For the targeted icy satellite encounters the primary instruments are IMS and ELS. Here we focus our discussion on the IMS. Papers describing the IMS in detail can be found in the works of McComas and Nordholt [1990], McComas et al. [1998], and Nordholt et al. [1998]. Basically, the IMS uses time-of-flight technology with cylindrical geometry providing an instantaneous 2-D view of the plasma over an angular range of $160^{\circ}$; the angular resolution is $8.3^{\circ} \times 20^{\circ}$. The energy resolution is $\Delta \mathrm{E} / \mathrm{E} \sim 17 \%$. The spectrometer has a medium mass resolution feature $(\mathrm{M} / \Delta \mathrm{M} \sim 8)$ called straight through, $\mathrm{ST}$, and a high mass resolution feature $(\mathrm{M} / \Delta \mathrm{M} \sim 60)$ using linear electric field technique, LEF, which can also be referred to as an isochronous (time focusing) technique. The present estimates of the IMS geometric factors are $\mathrm{G} \sim 10^{-3} \mathrm{~cm}^{2}$-ster-eV/eV for ST feature and $\mathrm{G} \sim 10^{-4} \mathrm{~cm}^{2}$-ster-eV/eV for LEF feature. We will assume these values for our simulations.

[13] The IMS (see Figure 1 from Nordholt et al. [1998]) is composed of a top hat toroidal analyzer for $\mathrm{E} / \mathrm{Q}$ selection, after which the ions are post-accelerated by $-15 \mathrm{kV}$. The ions then pass through $\sim 1 \mu \mathrm{gm} / \mathrm{cm}^{2}$ carbon foils after which they enter the time-of-flight (TOF) section of the IMS. The TOF section has a total of $\pm 15 \mathrm{kV}$ (i.e., $30 \mathrm{kV}$ ) across it to setup a linear electric field (LEF). Fragments can exit the carbon foils as neutrals, negative ions, and positive ions. The neutral fragments and negative fragments will strike the ST stop microchannel plate (MCP) and produce two peaks, on average, in the TOF spectrum. If they exit the carbon foil as positive ions, they will execute a harmonic oscillator motion in the LEF region of the TOF section and strike the LEF stop MCP. For incident atomic ions the LEF mass resolution will be very high $\mathrm{M} / \Delta \mathrm{M} \sim 60$, while for incident molecular ions the positive ion fragments will be shifted to lower LEF TOF relative to their atomic ion position and have peaks broader in LEF TOF relative to that for incident atomic ions (i.e., molecular fragment LEF TOF peaks will have lower mass resolution relative to that for atomic ions $(\mathrm{M} / \Delta \mathrm{M}<60))$; this results from the detuning characteristic of the LEF TOF section when the fragments exiting the carbon foil have exit energies lower than the incident molecular ion (i.e., the fragments retain the same speed as the incident molecular ion). For atomic ions with $\mathrm{E} / \mathrm{Q} \geq$ $17 \mathrm{kV}$ and exit the carbon foils as positive ions their trajectories cannot be turned upward to hit the LEF stop detector. Under these circumstances they will strike the ST detector, and we can have up to three ST peaks in the TOF spectrum (i.e., negative ion, neutral, and positive ion). At lower E/Q there will also be an LEF ghost peak in the ST spectrum, which results from the positive ion hitting the LEF stop MCP but not producing a pulse in the LEF MCP, and then the emission of secondary electrons emitted by the LEF MCP which are accelerated downward to strike the stop ST MCP. So, for E/Q $<17 \mathrm{kV}$ we can still have three peaks in the ST TOF spectrum (i.e., negative ion, neutral, and LEF ghost peak).

[14] CAPS also has an actuator which allows the IMS to have nearly $2 \pi$ coverage in about $3 \mathrm{~min}(1 \% \mathrm{~s}$ scan rate). During the targeted flybys with an equatorial pass we recommend the collimator plane to be in the equatorial plane of Saturn and have the actuator dither above and below this plane to look for pitch angle scattering of the pickup ions which are expected to have ring distributions as discussed.

[15] The relatively large geometric factor for CAPS allows it to detect ambient and pickup ions with high sensitivity. By combining ST and LEF measurements and using the mass deconvolution capabilities of the IMS Spectrum Analyzer Module (SAM) [see Sittler, 1993], the IMS will be able to resolve the water group ions (i.e., resolve $\mathrm{H}_{2} \mathrm{O}^{+}, \mathrm{OH}^{+}$, and $\mathrm{H}_{3} \mathrm{O}^{+}$from each other), organics such as $\mathrm{CH}_{4}^{+}, \mathrm{NH}_{3}^{+}$, molecular oxygen $\mathrm{O}_{2}^{+}$, ozone $\mathrm{O}_{3}^{+}$, and other species such as $\mathrm{CO}_{2}^{+}$and $\mathrm{MgO}^{+}$. For example, a standard M/Q analyzer would not be able to distinguish between $\mathrm{O}^{+}$and $\mathrm{CH}_{4}^{+}$, but because of the nonlinear characteristics of the LEF technique the $\mathrm{CH}_{4}^{+}$ion will breakup in the carbon foil and the fragment $\mathrm{C}^{+}$ion exiting the foil will appear near the atomic $\mathrm{C}^{+}$ion LEF TOF position but shifted in TOF enough to allow it to be resolved by the IMS. In the case where the fragments are of nearly equal mass, such as $\mathrm{O}_{2}^{+}$or $\mathrm{CO}_{2}^{+}$, then the fragments will have energies significantly less than the $-15 \mathrm{kV}$ post-acceleration of the IMS (i.e., $\mathrm{O}_{2}^{+} \rightarrow \mathrm{O}^{+}$the $\mathrm{O}^{+}$fragment will have energy $\sim 7.5 \mathrm{kV}$ ) so that the LEF mass peaks will have a large shift to lower TOF relative to that for its atomic ions $\left(\mathrm{O}_{2}^{+} \rightarrow \mathrm{O}^{+}\right.$ versus $\mathrm{O}^{+} \rightarrow \mathrm{O}^{+}$) and the peaks will be significantly broader than that for incident atomic ions (i.e., $M / \Delta \mathrm{M} \ll 60$ ). Also, because oxygen has a larger affinity to electrons when passing through carbon foils relative to that for carbon and nitrogen, it will be easier to detect carbon or nitrogen fragments relative to that for oxygen fragments. This will be helpful when it comes to detecting $\mathrm{NH}_{3}^{+}$and $\mathrm{CH}_{4}^{+}$using LEF data. Our simulations, which are based on calibration data, will demonstrate these effects.

[16] With the collimator in the equatorial plane and with an instrument energy-per-charge (E/Q) sweep time of $4 \mathrm{~s}$ $(1 \mathrm{eV} \leq \mathrm{E} / \mathrm{Q} \leq 50 \mathrm{keV}$ with logarithmically spaced $63 \mathrm{E} / \mathrm{Q}$ steps), CAPS should be able to provide $52 \mathrm{~km}$ spatial resolution of the pickup ion densities. Telemetry limitations confine our measurements of the dominant ambient and pickup ions to only seven ions. However, CAPS has the ability to alternate between seven different ion species after each energy sweep and thus provide the sampling up to 14 ions every $8 \mathrm{~s}$. In addition to the ion data (i.e., seven to 14 ion species with full energy and pitch angle coverage), the IMS will also provide TOF versus E/Q spectrograms for 
both ST and LEF data (i.e., data summed over 64 E/Q sweeps and collapsed in angle for high sensitivity so no angular information is available in this data set). This data set will allow us to detect both major and minor ions with very high sensitivity. However, the time resolution of this data set is about 4 minutes or an equivalent spatial resolution of about $3000 \mathrm{~km}$, which is much larger than the dimensions of either Dione or Enceladus. Therefore this data set provides no spatial information less than $3000 \mathrm{~km}$. We will present simulations of both data products in the form of 2-D energy-angle contours of ion count rate for dominant ion species and some minor ion species and 2-D $\mathrm{E} / \mathrm{Q}$ versus TOF contours of ion counts for all ion species used in our model calculations. The ion count rate data is referred to as A cycle data, while the E/Q versus TOF data is referred to as B cycle data. An A cycle data product is composed of $8 \mathrm{E} / \mathrm{Q}$ sweeps and for the highest data rate there will be eight A cycles per B cycle. Each A cycle is $32 \mathrm{~s}$ long, while a B cycle is $4 \mathrm{~min}$ long. For all targeted satellite flybys the CAPS telemetry rate will be at its maximum rate of $16 \mathrm{kbps}$.

\section{Model of Sputtered Atmospheres for Dione and Enceladus}

[17] In order to construct a model of the sputtered atmospheres for either Dione or Enceladus, one needs the total sputtering source rate and the energy spectrum of the sputtered neutrals. For simplicity we assume spherical symmetry, but before the actual targeted flybys we will consider models which include asymmetries due to ion Mach number, ion and electron pressure anisotropies, and spatial inhomogeneities of the satellite surfaces. The latter can be temperature maps, surface properties that the imagers might detect, and possible compositional spatial variations that VIMS might detect. A Monte Carlo calculation of the sputtered atmosphere including asymmetries caused by Saturn's gravitational field should also be carried out. However, to obtain preliminary estimates of the molecular species to be detected by CAPS we will use a simple spherically symmetric formula given by Johnson [1990] [see also Watson, 1982; Sieveka, 1983]. For this calculation we use an energy spectrum for the sputtered neutrals of the form, $f(E, \cos \theta)=4 U E \cos \theta /(E+U)^{3}$, where $f(E, \cos \theta)$ is the normalized ejecta distribution with $\mathrm{E}$ the exit energy, $\theta$ is the exit direction, and $U$ is a parameter determined from measurements [Johnson, 1990, 1998]. The ejecta is presumed to be predominantly neutrals. The effect of the small component of directly sputtered ions will be considered in subsequent work [see Madey et al., 2002].

[18] For our source term $\mathrm{S}$ we will use the maximum sputtered flux estimated by Jurac et al. [2001a, 2001b] for both Dione and Enceladus. In their calculations they included the enhancements due to finite gyroradius effects (i.e., effective satellite area) and angular enhancement caused by the $\sec \varphi$ dependence of the sputtered flux, where $\varphi$ is the angle of incidence of the impacting ion relative to the normal of the satellites surface. Jurac et al. [2001a, 2001b] also included the photo sputter flux term. Because the electron-induced sputtering [Madey et al., 2002], which primarily contributes to decomposition [Sieger et al., 1998], was not estimated, these are lower bounds to the yields
[Johnson et al., 2004a, 2004b]. With the above caveats, the sputter satellite source rates are approximately $\mathrm{S}_{\mathrm{D}}=9.6 \times$ $10^{25} \mathrm{~mol} / \mathrm{s}$ and $\mathrm{S}_{\mathrm{E}}=2.1 \times 10^{25} \mathrm{~mol} / \mathrm{s}$ for Dione and Enceladus, respectively.

[19] Our sputtered atmospheric model assumes [Jurac et al., 2001a, 2001b] that the incident ion flux is dominated by heavies (i.e., $\mathrm{O}^{+}$) and that the dominant sputtered neutral is water molecules. We will include additional species in our model atmosphere: $\mathrm{H}_{2}, \mathrm{O}_{2}, \mathrm{CO}_{2}$, and $\mathrm{NH}_{3}$ each at the $10 \%$ level relative to the sputtered flux for water. $\mathrm{H}_{2}$ and $\mathrm{O}_{2}$ are direct sputter products due to energetic ion decomposition of ice [Johnson, 1990; Johnson et al., 2004b]. $\mathrm{CO}_{2}$ has been seen by the Galileo spacecraft to be an atmospheric constituent at Callisto [Carlson, 1999] and $\mathrm{CO}_{2}$ has been seen at the Uranian satellite Ariel [Grundy et al., 2002]. $\mathrm{NH}_{3}$ has been suggested as a volatile in the Saturnian satellites because of Titan [Stevenson, 1982]. Since the ejection of trace species is temperature dependent [Johnson, 1990; Johnson et al., 2004b; Brown et al., 1982] and depends on the satellite composition, the choice of $10 \%$ is for convenience. For example, abundance of the minor species such as $\mathrm{CH}_{4}$ and $\mathrm{NH}_{3}$ could be lower at the few percent level. These results can then be scaled based on more detailed knowledge or new observations. However, the results given here should allow the reader to determine the ability of CAPS to detect the minor species for either of the targeted flybys. We could also have included $\mathrm{CH}_{4}$, but since the surfaces are oxidizing [Johnson et al., 2004a, 2004 b], we felt that having $\mathrm{CO}_{2}$ was more appropriate.

[20] In Figures 1 and 2 we show the result of our model calculations for Dione and Enceladus, respectively, which shows neutral density plotted as a function of height relative to the surface. In these calculations we used $\mathrm{U}=0.055 \mathrm{eV}$ for $\mathrm{H}_{2} \mathrm{O}, \mathrm{CO}_{2}$, and $\mathrm{NH}_{3}$, while for $\mathrm{H}_{2}$ and $\mathrm{O}_{2}$ we use $\mathrm{U}=$ $0.01 \mathrm{eV}$ [Johnson et al., 1998]. For both bodies the maximum water molecule density is about $10^{5} \mathrm{~mol} / \mathrm{cm}^{3}$ at their surfaces. In the case of Dione the next most important neutral near the surface is $\mathrm{O}_{2}$, while far from Dione, in order of importance, we have $\mathrm{CO}_{2}, \mathrm{NH}_{3}$, and $\mathrm{H}_{2}$. In the case of Enceladus, $\mathrm{O}_{2}$ dominates relative to all the other minor species for all heights. For the remaining minor species in order of importance, we have $\mathrm{CO}_{2}, \mathrm{NH}_{3}$, and $\mathrm{H}_{2}$. To some degree the variation of the neutral atmospheric density with height can be traced to the assumed energy spectrum of the sputtered neutrals and the parameter U. With $4 \mathrm{~s}$ time resolution, CAPS should be able to measure the height variation of the neutrals from the pickup ion measurements which will then allow us to estimate $U$ for the different neutral species. Deviations from spherical symmetry are expected for radial distances $\sim$ Hill Lagrange Spheres, which has radii $5.7 \mathrm{R}_{\text {Dione }}$ (i.e., $3192 \mathrm{~km}$ ) for Dione and $3.4 \mathrm{R}_{\text {Enceladus }}$ (i.e., $850 \mathrm{~km}$ ) for Enceladus. For this paper we will ignore these corrections, which are easily included in full Monte Carlo calculations.

\section{Estimates of Pickup Ion Densities as a Function of Height for Dione and Enceladus}

\subsection{Wake Pass}

[21] In Figure 3 we show an assumed targeted flyby geometry where the spacecraft passes through the satellite's corotational wake at an unspecified height $\mathrm{H}=\left(\alpha-\mathrm{r}_{0}\right)$ at 


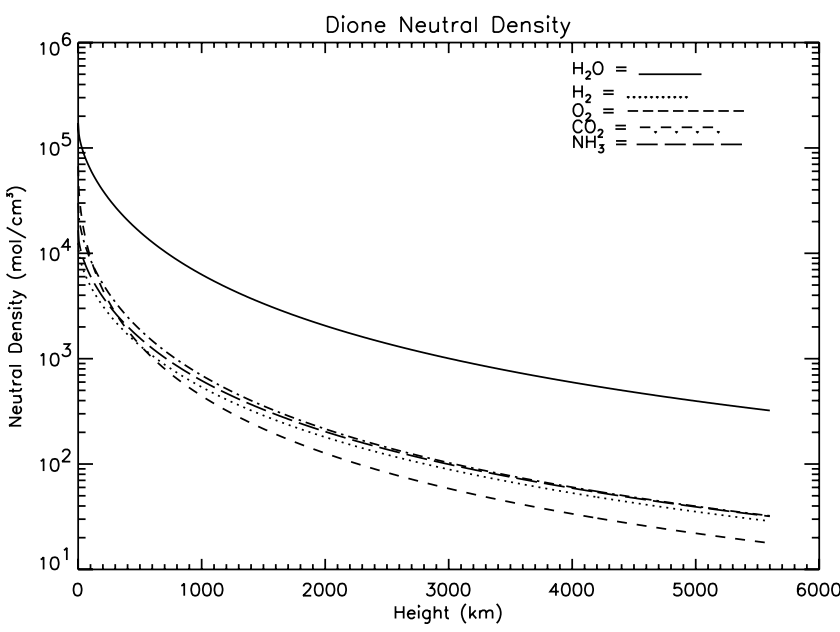

Figure 1. Computed neutral densities of Dione's sputtered atmosphere as a function of height. Assumed species are $\mathrm{H}_{2} \mathrm{O}, \mathrm{H}_{2}, \mathrm{O}_{2}, \mathrm{CO}_{2}$, and $\mathrm{NH}_{3}$. The latter four species are minor and assumed to each be $10 \%$ of $\mathrm{H}_{2} \mathrm{O}$ separately for the purposes of this study. This allows easy scaling as CAPS data becomes available. Since the atmospheres are collisionless, each species is treated separately. As discussed in the text, $\mathrm{H}_{2}$ and $\mathrm{O}_{2}$ are decomposition products due to radiolysis of $\mathrm{H}_{2} \mathrm{O}$ and $\mathrm{CO}_{2}$ and $\mathrm{NH}_{3}$ are examples of possible minor species suggested by satellite formation scenarios. Other species are clearly possible due to ion implantation, micrometeoroids, etc.

closest approach. The reduction of this distance will enhance CAPS ability to detect the pickup ions, since they will have less time to pitch angle scatter and spread out in phase space [Wu and Davidson, 1972; Hartle and Wu, 1973]. Pitch angle scattering will tend to transform a ring distribution into a shell distribution [Vasyliunas and Siscoe, 1976]. This spreads the ions in velocity space and makes the measured fluxes lower at a particular energy. Also, pitch angle scattering will tend to make the ions move along the magnetic field line and thus dilute the pickup ion flux in coordinate space. The net effect is to reduce our ability to distinguish the newly produced pickup ions. A height for a wake pass of $\sim 500 \mathrm{~km}$ is optimal for Cassini because of the spacecraft's ability to turn in order to remove smearing effects when imaging the surface but is still close enough to measure ring distributions using CAPS. The figure also shows a fluid element as it passes through the satellite's atmosphere to the observation point where the spacecraft is located. As the fluid element moves through the atmosphere, it will collect pickup ions as the neutrals are ionized by charge exchange (Table 1), electron impact (Table 2), and photons (Table 3 ). In order to compute the pickup ion densities correctly we will also need estimates of the ambient densities and temperatures of the ions and electrons in the vicinity of Dione and Enceladus. These are given in Table 4. Table 4 also gives the ambient densities of the various neutral components at Dione and Enceladus. All the numbers in Table 4 were derived from the papers by Richardson et al. [1986, 1998]. Since our model calculations use a fluid approximation, we are ignoring the finite gyroradius effects of the pickup ions, as mentioned earlier. The gyroradius for $\mathrm{H}_{2} \mathrm{O}^{+}$at Dione is about $123 \mathrm{~km}$, which is

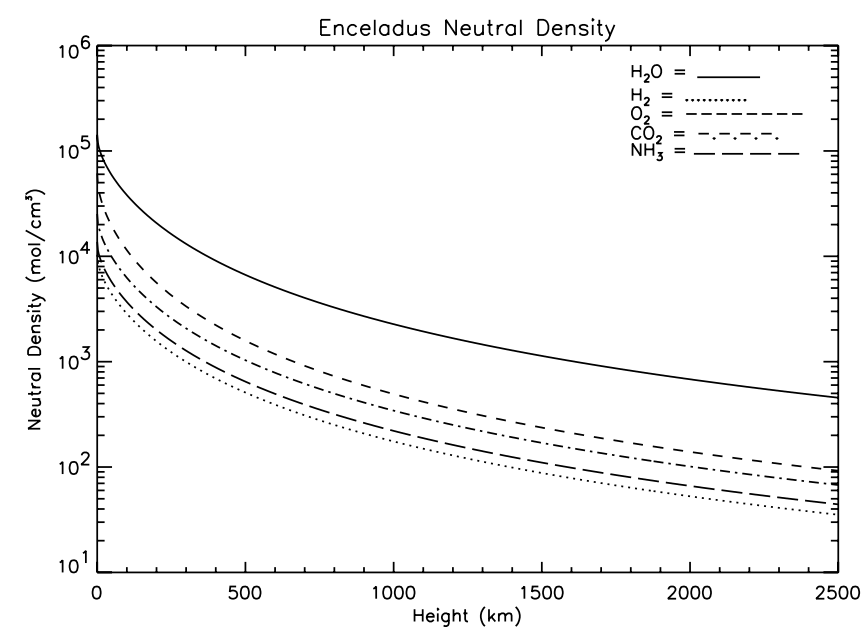

Figure 2. Same as Figure 1 except for Enceladus.

small relative to its radius $(560 \mathrm{~km})$, and at Enceladus is about $20 \mathrm{~km}$, which is much less than the radius of Enceladus $(250 \mathrm{~km})$. So, to a good approximation, the fluid approximation should be accurate. Deviations from this will occur on the side facing Saturn where the pickup ions can gyrate into the body's surface for heights less than the ion gyroradius. So, for heights near the satellites surface we would expect to see an asymmetry in the observed pickup ion densities. From Richardson [1986] the observed plasma velocity at Dione is about $46 \mathrm{~km} / \mathrm{s}$ and at Enceladus is

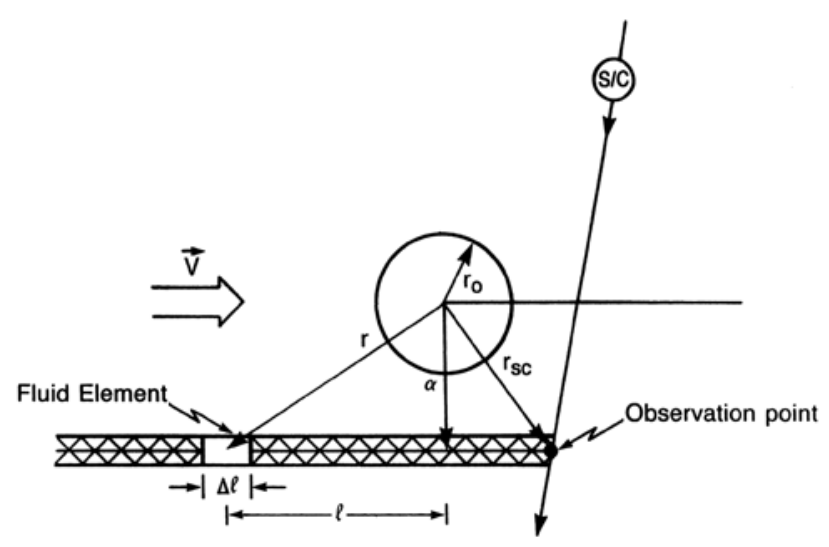

Figure 3. Shows encounter geometry and how we compute pickup ion densities as a fluid element passes through the satellites sputtered atmosphere and picks up ions as the neutrals are ionized due to photoionization, electron impact ionization, and charge exchange reactions. This model assumes the atmosphere is collisionally thin and a strong interaction does not occur. We also neglect finite gyroradius corrections. S/C stands for spacecraft. Script 1 signifies the integrated line-of-sight distance of the fluid element and $\Delta \mathrm{l}$ is the differential width of the fluid element; $\alpha$ is the impact parameter of the fluid element. The parameter $r_{0}$ is the body radius, $r_{s / c}$ is the radial distance of the spacecraft from the center of the body, and $\mathrm{r}$ is the radial distance of the fluid element from the center of the body. $\vec{V}$ is the flow velocity of the fluid element moving in the corotation direction. 
Table 1. Charge Exchange Rates in $\mathrm{cm}^{3} / \mathrm{s}$

\begin{tabular}{|c|c|c|c|}
\hline Reaction & Enceladus & Dione & Reference \\
\hline $\mathrm{H}^{+}+\mathrm{H} \rightarrow \mathrm{H}+\mathrm{H}^{+}$ & $1.5 \mathrm{E}-8$ & $1.5 \mathrm{E}-8$ & Newman et al. [1982] \\
\hline $\mathrm{O}^{+}+\mathrm{H} \rightarrow \mathrm{O}+\mathrm{H}^{+}$ & $6.2 \mathrm{E}-9$ & $6.3 \mathrm{E}-9$ & $\begin{array}{l}\text { Stebbings and } \\
\quad \text { Rutherford [1968] }\end{array}$ \\
\hline $\mathrm{O}^{+}+\mathrm{OH} \rightarrow \mathrm{O}_{2}+\mathrm{H}^{+}$ & $1.2 \mathrm{E}-10$ & $1.2 \mathrm{E}-10$ & $\begin{array}{l}\text { Giguere and } \\
\text { Huebner [1978] }\end{array}$ \\
\hline $\mathrm{H}^{+}+\mathrm{H}_{2} \rightarrow \mathrm{H}+\mathrm{H}_{2}^{+}$ & $1.7 \mathrm{E}-10$ & $1.7 \mathrm{E}-10$ & Tawara $[1978]$ \\
\hline $\mathrm{H}_{2}^{+}+\mathrm{H}_{2} \rightarrow \mathrm{H}_{2}+\mathrm{H}_{2}^{+}$ & $6.6 \mathrm{E}-9$ & $6.6 \mathrm{E}-9$ & $\begin{array}{l}\text { Massey and } \\
\quad \text { Gilbody [1974] }\end{array}$ \\
\hline $\mathrm{H}^{+}+\mathrm{H}_{2} \mathrm{O} \rightarrow \mathrm{H}+\mathrm{H}_{2} \mathrm{O}^{+}$ & $5.0 \mathrm{E}-8$ & $5.0 \mathrm{E}-8$ & Tawara $[1978]$ \\
\hline $\mathrm{H}^{+}+\mathrm{H}_{2} \mathrm{O} \rightarrow \mathrm{H}+\mathrm{H}_{2} \mathrm{O}^{+}$ & $8.2 \mathrm{e}-9$ & $8.2 \mathrm{e}-9$ & Ip [1997] \\
\hline $\mathrm{O}^{+}+\mathrm{H}_{2} \mathrm{O} \rightarrow \mathrm{O}+\mathrm{H}_{2} \mathrm{O}^{+}$ & 2.3E-9 & 2.3E-9 & Albritton [1978] \\
\hline $\mathrm{OH}^{+}+\mathrm{H}_{2} \mathrm{O} \rightarrow \mathrm{OH}+\mathrm{H}_{2} \mathrm{O}^{+}$ & $1.6 \mathrm{E}-9$ & $1.6 \mathrm{E}-9$ & $\begin{array}{l}\text { Huntress [1977], } \\
\quad \text { Kim et al. }[1975]\end{array}$ \\
\hline $\mathrm{OH}^{+}+\mathrm{H}_{2} \rightarrow \mathrm{H}+\mathrm{H}_{2} \mathrm{O}^{+}$ & $1.1 \mathrm{E}-9$ & $1.1 \mathrm{E}-9$ & Huntress [1977] \\
\hline $\mathrm{H}_{2}^{+}+\mathrm{H}_{2} \mathrm{O} \rightarrow \mathrm{H}_{2}+\mathrm{H}_{2} \mathrm{O}^{+}$ & 3.9E-9 & $3.9 \mathrm{E}-9$ & Huntress [1977] \\
\hline $\mathrm{OH}^{+}+\mathrm{OH} \rightarrow \mathrm{H}_{2} \mathrm{O}^{+}+\mathrm{O}$ & $7.0 \mathrm{e}-10$ & $7.0 \mathrm{e}-10$ & $I p$ [1997] \\
\hline $\mathrm{H}_{2}^{+}+\mathrm{OH} \rightarrow \mathrm{H}_{2} \mathrm{O}^{+}+\mathrm{H}$ & $7.6 \mathrm{e}-10$ & $7.6 \mathrm{e}-10$ & Ip [1997] \\
\hline $\mathrm{H}^{+}+\mathrm{O} \rightarrow \mathrm{H}+\mathrm{O}^{+}$ & $5.5 \mathrm{E}-9$ & $5.5 \mathrm{E}-9$ & $\begin{array}{l}\text { Stebbings et al. } \\
\text { [1964] }\end{array}$ \\
\hline $\mathrm{O}^{+}+\mathrm{O} \rightarrow \mathrm{O}+\mathrm{O}^{+}$ & 9.9E-9 & $9.9 \mathrm{E}-9$ & $\begin{array}{l}\text { Stebbings et al. } \\
{[1964]}\end{array}$ \\
\hline $\mathrm{O}_{2}^{+}+\mathrm{O}_{2} \rightarrow \mathrm{O}_{2}+\mathrm{O}_{2}^{+}$ & $6.4 \mathrm{E}-9$ & $6.4 \mathrm{E}-9$ & $\begin{array}{l}\text { Banks and } \\
\quad \text { Kockarts [1973] }\end{array}$ \\
\hline $\mathrm{H}_{2}^{+}+\mathrm{O}_{2} \rightarrow \mathrm{H}_{2}+\mathrm{O}_{2}^{+}$ & 9.4E-9 & $9.4 \mathrm{E}-9$ & Tawara $[1978]$ \\
\hline $\mathrm{O}^{+}+\mathrm{O}_{2} \rightarrow \mathrm{O}+\mathrm{O}_{2}^{+}$ & 2.1E-9 & $2.1 \mathrm{E}-9$ & $\begin{array}{l}\text { Albritton et al. } \\
\text { [1977] }\end{array}$ \\
\hline $\mathrm{OH}^{+}+\mathrm{O}_{2} \rightarrow \mathrm{OH}+\mathrm{O}_{2}^{+}$ & $2.0 \mathrm{E}-10$ & $2.0 \mathrm{E}-10$ & Bortner et al. [1972] \\
\hline $\mathrm{H}_{2} \mathrm{O}^{+}+\mathrm{O}_{2} \rightarrow \mathrm{H}_{2} \mathrm{O}+\mathrm{O}_{2}^{+}$ & $5.0 \mathrm{E}-9$ & $5.0 \mathrm{E}-9$ & $\begin{array}{l}\text { Fehsenfeld } \\
\quad \text { et al. }[1967]\end{array}$ \\
\hline $\mathrm{H}^{+}+\mathrm{O}_{2} \rightarrow \mathrm{H}+\mathrm{O}_{2}^{+}$ & $1.17 \mathrm{E}-9$ & $1.17 \mathrm{E}-9$ & Rudd et al. [1985] \\
\hline $\mathrm{O}^{+}+\mathrm{CO}_{2} \rightarrow \mathrm{CO}+\mathrm{O}_{2}^{+}$ & $1.1 \mathrm{E}-9$ & $1.1 \mathrm{E}-9$ & $\begin{array}{l}\text { Giguere and } \\
\text { Huebner [1978] }\end{array}$ \\
\hline $\mathrm{H}^{+}+\mathrm{OH} \rightarrow \mathrm{H}+\mathrm{OH}^{+}$ & $3.0 \mathrm{E}-10$ & $3.0 \mathrm{E}-10$ & $\begin{array}{l}\text { Giguere and } \\
\text { Huebner [1978] }\end{array}$ \\
\hline $\mathrm{H}^{+}+\mathrm{OH} \rightarrow \mathrm{H}+\mathrm{OH}^{+}$ & $2.1 \mathrm{e}-9$ & $2.1 \mathrm{e}-9$ & $I p[1997]$ \\
\hline $\mathrm{H}_{2}^{+}+\mathrm{O} \rightarrow \mathrm{H}+\mathrm{OH}^{+}$ & $1.0 \mathrm{E}-9$ & $1.0 \mathrm{E}-9$ & $\begin{array}{l}\text { Giguere and } \\
\text { Huebner [1978] }\end{array}$ \\
\hline $\mathrm{O}^{+}+\mathrm{H}_{2} \rightarrow \mathrm{H}+\mathrm{OH}^{+}$ & $1.6 \mathrm{E}-9$ & $1.6 \mathrm{E}-9$ & $\begin{array}{l}\text { Huntress }[1977], \\
\quad \text { Kim et al. }[1975]\end{array}$ \\
\hline $\mathrm{H}_{2}^{+}+\mathrm{OH} \rightarrow \mathrm{OH}^{+}+\mathrm{H}_{2}$ & $7.6 \mathrm{e}-10$ & $7.6 \mathrm{e}-10$ & Ip [1997] \\
\hline $\mathrm{O}^{+}+\mathrm{OH} \rightarrow \mathrm{O}+\mathrm{OH}^{+}$ & $3.0 \mathrm{E}-10$ & $3.0 \mathrm{E}-10$ & $\begin{array}{l}\text { Giguere and } \\
\text { Huebner [1978] }\end{array}$ \\
\hline $\mathrm{O}^{+}+\mathrm{OH} \rightarrow \mathrm{O}+\mathrm{OH}^{+}$ & $3.6 \mathrm{e}-10$ & $3.6 \mathrm{e}-10$ & Ip [1997] \\
\hline $\mathrm{O}^{+}+\mathrm{NH}_{3} \rightarrow \mathrm{NH}_{2}+\mathrm{OH}^{+}$ & $2.2 \mathrm{E}-9$ & $2.2 \mathrm{E}-9$ & $\begin{array}{l}\text { Huntress [1977], } \\
\text { Oppenheimer } \\
{[1975]^{\mathrm{a}}}\end{array}$ \\
\hline $\mathrm{O}^{+}+\mathrm{CO}_{2} \rightarrow \mathrm{O}+\mathrm{CO}_{2}^{+}$ & $1.1 \mathrm{E}-10$ & $1.1 \mathrm{E}-10$ & Rudd et al. $[1985]^{\mathrm{a}}$ \\
\hline $\mathrm{H}^{+}+\mathrm{CO}_{2} \rightarrow \mathrm{H}+\mathrm{CO}_{2}^{+}$ & $1.1 \mathrm{E}-9$ & $1.1 \mathrm{E}-9$ & Rudd et al. [1985] \\
\hline $\mathrm{H}^{+}+\mathrm{NH}_{3} \rightarrow \mathrm{H}+\mathrm{NH}_{3}^{+}$ & $5.2 \mathrm{E}-9$ & $5.2 \mathrm{E}-9$ & Huntress [1977] \\
\hline $\mathrm{H}_{2}^{+}+\mathrm{NH}_{3} \rightarrow \mathrm{H}_{2}+\mathrm{NH}_{3}^{+}$ & $5.7 \mathrm{E}-9$ & 5.7E-9 & Huntress [1977] \\
\hline $\mathrm{OH}^{+}+\mathrm{NH}_{3} \rightarrow \mathrm{OH}+\mathrm{NH}_{3}^{+}$ & $1.2 \mathrm{E}-9$ & $1.2 \mathrm{E}-9$ & Huntress [1977] \\
\hline $\begin{array}{l}\mathrm{H}_{2} \mathrm{O}^{+}+\mathrm{NH}_{3} \rightarrow \\
\mathrm{H}_{2} \mathrm{O}+\mathrm{NH}_{3}^{+}\end{array}$ & $2.2 \mathrm{E}-9$ & $2.2 \mathrm{E}-9$ & Huntress [1977] \\
\hline $\mathrm{H}_{2}^{+}+\mathrm{H}_{2} \mathrm{O} \rightarrow \mathrm{H}_{2}+\mathrm{H}_{3} \mathrm{O}^{+}$ & 3.4E-9 & $3.4 \mathrm{E}-9$ & Huntress [1977] \\
\hline $\mathrm{OH}^{+}+\mathrm{H}_{2} \mathrm{O} \rightarrow \mathrm{O}+\mathrm{H}_{3} \mathrm{O}^{+}$ & $1.3 \mathrm{E}-9$ & $1.3 \mathrm{E}-9$ & Huntress [1977] \\
\hline $\mathrm{H}_{2} \mathrm{O}^{+}+\mathrm{H}_{2} \rightarrow \mathrm{H}+\mathrm{H}_{3} \mathrm{O}^{+}$ & $6.1 \mathrm{E}-10$ & $6.1 \mathrm{E}-10$ & $\begin{array}{l}\text { Huntress [1977], } \\
\quad \text { Kim et al. }[1975]\end{array}$ \\
\hline $\mathrm{H}_{2} \mathrm{O}^{+}+\mathrm{H}_{2} \mathrm{O} \rightarrow \mathrm{OH}+\mathrm{H}_{3} \mathrm{O}^{+}$ & $2.1 \mathrm{E}-9$ & $2.1 \mathrm{E}-9$ & Huntress [1977] \\
\hline
\end{tabular}

${ }^{\mathrm{a}}$ Estimated.

$33 \mathrm{~km} / \mathrm{s}$. The magnetic field strength at Dione is about 70 $\mathrm{nT}$, while that at Enceladus is $312 \mathrm{nT}$ [Connerney et al., 1983].

[22] The results of our pickup ion densities as a function of height for a wake pass is shown in Figures $4 a$ and $4 b$ for Dione and Figures 5a and 5b for Enceladus. We arbitrarily set the closest approach distance equal to the satellite radius
Table 2. Electron Impact Ionization Rates in $\mathrm{cm}^{3} / \mathrm{s}$

\begin{tabular}{|c|c|c|c|}
\hline Reaction & Enceladus & Dione & References \\
\hline $\mathrm{H}+\mathrm{e} \rightarrow \mathrm{H}^{+}+2 \mathrm{e}$ & $1.3 \mathrm{E}-10$ & $2.45 \mathrm{E}-9$ & Lotz [1967] \\
\hline $\mathrm{H}+\mathrm{e}^{\mathrm{a}} \rightarrow \mathrm{H}^{+}+2 \mathrm{e}$ & $3.1 \mathrm{E}-8$ & $3.1 \mathrm{E}-8$ & Lotz [1967] \\
\hline $\mathrm{H}_{2}+\mathrm{e} \rightarrow \mathrm{H}^{+}+\mathrm{H}+2 \mathrm{e}$ & 7.3E-14 & $3.3 \mathrm{E}-11$ & Ip [1997] \\
\hline $\mathrm{H}_{2}+\mathrm{e}^{\mathrm{a}} \rightarrow \mathrm{H}^{+}+\mathrm{H}+2 \mathrm{e}$ & $1.01 \mathrm{E}-8$ & $1.92 \mathrm{E}-8$ & $\operatorname{Ip}[1997]$ \\
\hline $\begin{array}{l}\mathrm{H}_{2} \mathrm{O}+\mathrm{e} \rightarrow \\
\mathrm{H}^{+}+\mathrm{OH}+2 \mathrm{e}\end{array}$ & $1.66 \mathrm{E}-12$ & 7.4E-11 & $\begin{array}{l}\text { Orient and } \\
\quad \text { Srivastava [1987] }\end{array}$ \\
\hline $\begin{array}{l}\mathrm{H}_{2} \mathrm{O}+\mathrm{e}^{\mathrm{a}} \rightarrow \\
\mathrm{H}^{+}+\mathrm{OH}+2 \mathrm{e}\end{array}$ & $4.06 \mathrm{E}-8$ & $4.35 \mathrm{E}-8$ & $\begin{array}{l}\text { Orient and } \\
\quad \text { Srivastava [1987] }\end{array}$ \\
\hline $\begin{array}{l}\mathrm{NH}_{3}+\mathrm{e} \rightarrow \\
\mathrm{H}^{+}+\mathrm{NH}_{2}+2 \mathrm{e}\end{array}$ & $2.58 \mathrm{E}-14$ & $1.79 \mathrm{E}-12$ & Mark et al. [1977] \\
\hline $\begin{array}{l}\mathrm{NH}_{3}+\mathrm{e}^{\mathrm{a}} \rightarrow \\
\mathrm{H}^{+}+\mathrm{NH}_{2}+2 \mathrm{e}\end{array}$ & $5.28 \mathrm{E}-10$ & $5.44 \mathrm{E}-10$ & Mark et al. [1977] \\
\hline $\mathrm{H}_{2}+\mathrm{e} \rightarrow \mathrm{H}_{2}^{+}+2 \mathrm{e}$ & $2.26 \mathrm{E}-10$ & $2.2 \mathrm{E}-9$ & Kieffer [1969] \\
\hline $\mathrm{H}_{2}+\mathrm{e}^{\mathrm{a}} \rightarrow \mathrm{H}_{2}^{+}+2 \mathrm{e}$ & $5.5 \mathrm{E}-8$ & $5.5 \mathrm{E}-8$ & Kieffer [1969] \\
\hline $\begin{array}{l}\mathrm{NH}_{3}+\mathrm{e} \rightarrow \\
\quad \mathrm{NH}+\mathrm{H}_{2}^{+}+2 \mathrm{e}\end{array}$ & $1.35 \mathrm{E}-13$ & $1.86 \mathrm{E}-12$ & Mark et al. [1977] \\
\hline $\begin{array}{l}\mathrm{NH}_{3}+\mathrm{e}^{\mathrm{a}} \underset{ }{\rightarrow} \\
\mathrm{NH}+\mathrm{H}_{2}^{+}+2 \mathrm{e}\end{array}$ & $9.51 \mathrm{E}-11$ & $9.63 \mathrm{E}-11$ & Mark et al. [1977] \\
\hline $\begin{array}{l}\mathrm{H}_{2} \mathrm{O}+\mathrm{e} \rightarrow \\
\mathrm{H}_{2} \mathrm{O}^{+}+2 \mathrm{e}\end{array}$ & $8.6 \mathrm{E}-11$ & $8.88 \mathrm{E}-10$ & $\begin{array}{l}\text { Orient and } \\
\quad \text { Srivastava [1987] }\end{array}$ \\
\hline $\begin{array}{l}\mathrm{H}_{2} \mathrm{O}+\mathrm{e}^{\mathrm{a}} \rightarrow \\
\mathrm{H}_{2} \mathrm{O}^{+}+2 \mathrm{e}\end{array}$ & $9.32 \mathrm{E}-8$ & $9.3 \mathrm{E}-8$ & $\begin{array}{l}\text { Orient and } \\
\quad \text { Srivastava [1987] }\end{array}$ \\
\hline $\mathrm{O}+\mathrm{e} \rightarrow \mathrm{O}^{+}+2 \mathrm{e}$ & $2.01 \mathrm{E}-10$ & 2.0E-9 & Lotz [1967] \\
\hline $\mathrm{O}+\mathrm{e}^{\mathrm{a}} \rightarrow \mathrm{O}^{+}+2 \mathrm{e}$ & $8.2 \mathrm{E}-8$ & 8.2E-8 & Lotz [1967] \\
\hline $\begin{array}{l}\mathrm{H}_{2} \mathrm{O}+\mathrm{e} \rightarrow \\
\quad \mathrm{O}^{+}+\mathrm{OH}+2 \mathrm{e}\end{array}$ & $1.26 \mathrm{E}-13$ & $9.72 \mathrm{E}-12$ & $\begin{array}{l}\text { Orient and } \\
\quad \text { Srivastava [1987] }\end{array}$ \\
\hline $\begin{array}{l}\mathrm{H}_{2} \mathrm{O}+\mathrm{e}^{\mathrm{a}} \rightarrow \\
\mathrm{O}^{+}+\mathrm{OH}+2 \mathrm{e}\end{array}$ & $1.06 \mathrm{E}-8$ & $1.16 \mathrm{E}-8$ & $\begin{array}{l}\text { Orient and } \\
\quad \text { Srivastava [1987] }\end{array}$ \\
\hline $\begin{array}{l}\mathrm{CO}_{2}+\mathrm{e} \rightarrow \\
\mathrm{O}^{+}+\mathrm{CO}+2 \mathrm{e}\end{array}$ & $2.17 \mathrm{E}-12$ & 7.4E-11 & $\begin{array}{l}\text { Orient and } \\
\quad \text { Srivastava [1987] }\end{array}$ \\
\hline $\begin{array}{l}\mathrm{CO}_{2}+\mathrm{e}^{\mathrm{a}} \rightarrow \\
\mathrm{O}^{+}+\mathrm{CO}+2 \mathrm{e}\end{array}$ & $1.22 \mathrm{E}-8$ & $1.45 \mathrm{E}-8$ & $\begin{array}{l}\text { Orient and } \\
\quad \text { Srivastava [1987] }\end{array}$ \\
\hline $\mathrm{O}_{2}+\mathrm{e} \rightarrow \mathrm{O}_{2}^{+}+2 \mathrm{e}$ & $2.1 \mathrm{E}-10$ & $1.98 \mathrm{E}-9$ & $\begin{array}{l}\text { Banks and } \\
\quad \text { Kockarts }\end{array}$ \\
\hline $\mathrm{O}_{2}+\mathrm{e}^{\mathrm{a}} \rightarrow \mathrm{O}_{2}^{+}+2 \mathrm{e}$ & $1.5 \mathrm{E}-7$ & $1.72 \mathrm{E}-7$ & $\begin{array}{l}\text { Banks and } \\
\text { Kockarts [1973] }\end{array}$ \\
\hline $\mathrm{OH}+\mathrm{e} \rightarrow \mathrm{OH}^{+}+2 \mathrm{e}$ & $3.7 \mathrm{E}-10$ & $3.16 \mathrm{E}-9$ & $\begin{array}{l}\text { Richardson } \\
\quad \text { et al. }[1986]\end{array}$ \\
\hline $\mathrm{H}_{2} \mathrm{O}+\mathrm{e} \rightarrow \mathrm{OH}^{+}+2 \mathrm{e}$ & $1.7 \mathrm{E}-11$ & 3.7E-10 & $\begin{array}{l}\text { Orient and } \\
\quad \text { Srivastava [1987] }\end{array}$ \\
\hline $\mathrm{H}_{2} \mathrm{O}+\mathrm{e}^{\mathrm{a}} \rightarrow \mathrm{OH}^{+}+2 \mathrm{e}$ & $4.61 \mathrm{E}-8$ & $4.6 \mathrm{E}-8$ & $\begin{array}{l}\text { Orient and } \\
\quad \text { Srivastava [1987] }\end{array}$ \\
\hline $\mathrm{CO}_{2}+\mathrm{e} \rightarrow \mathrm{CO}_{2}^{+}+2 \mathrm{e}$ & $1.73 \mathrm{E}-10$ & $2.36 \mathrm{E}-9$ & $\begin{array}{l}\text { Orient and } \\
\quad \text { Srivastava [1987] }\end{array}$ \\
\hline $\mathrm{CO}_{2}+\mathrm{e}^{\mathrm{a}} \rightarrow \mathrm{CO}_{2}^{+}+2 \mathrm{e}$ & $1.63 \mathrm{E}-7$ & $1.9 \mathrm{E}-7$ & $\begin{array}{l}\text { Orient and } \\
\quad \text { Srivastava [1987] }\end{array}$ \\
\hline $\mathrm{CO}_{2}+\mathrm{e} \rightarrow \mathrm{CO}^{+}+2 \mathrm{e}$ & $4.45 \mathrm{E}-12$ & $7.05 \mathrm{E}-11$ & $\begin{array}{l}\text { Orient and } \\
\quad \text { Srivastava [1987] }\end{array}$ \\
\hline $\mathrm{CO}_{2}+\mathrm{e}^{\mathrm{a}} \rightarrow \mathrm{CO}^{+}+2 \mathrm{e}$ & $6.04 \mathrm{E}-9$ & $6.99 \mathrm{E}-9$ & $\begin{array}{l}\text { Orient and } \\
\quad \text { Srivastava [1987] }\end{array}$ \\
\hline $\begin{array}{l}\mathrm{CO}_{2}+\mathrm{e} \rightarrow \\
\mathrm{C}^{+}+\mathrm{O}_{2}+2 \mathrm{e}\end{array}$ & $9.5 \mathrm{E}-14$ & $5.92 \mathrm{E}-12$ & $\begin{array}{l}\text { Orient and } \\
\quad \text { Srivastava [1987] }\end{array}$ \\
\hline $\begin{array}{l}\mathrm{CO}_{2}+\mathrm{e}^{\mathrm{a}} \rightarrow \\
\mathrm{C}^{+}+\mathrm{O}_{2}+2 \mathrm{e}\end{array}$ & 3.33E-9 & $3.86 \mathrm{E}-9$ & $\begin{array}{l}\text { Orient and } \\
\quad \text { Srivastava [1987] }\end{array}$ \\
\hline $\mathrm{NH}_{3}+\mathrm{e} \rightarrow \mathrm{NH}_{3}^{+}+2 \mathrm{e}$ & & 2.03E-9 & Mark et al. [1977] \\
\hline $\mathrm{NH}_{3}+\mathrm{e}^{\mathrm{a}} \rightarrow \mathrm{NH}_{3}^{+}+2 \mathrm{e}$ & $5.23 \mathrm{E}-8$ & $5.26 \mathrm{E}-8$ & Mark et al. [1977] \\
\hline $\begin{array}{l}\mathrm{NH}_{3}+\mathrm{e} \rightarrow \\
\mathrm{NH}_{2}^{+}+\mathrm{H}+2 \mathrm{e}\end{array}$ & $3.85 \mathrm{E}-11$ & $6.6 \mathrm{E}-10$ & Mark et al. [1977] \\
\hline $\begin{array}{l}\mathrm{NH}_{3}+\mathrm{e}^{\mathrm{a}} \rightarrow \\
\mathrm{NH}_{2}^{+}+\mathrm{H}+2 \mathrm{e}\end{array}$ & 4.2E-8 & $4.32 \mathrm{E}-8$ & Mark et al. [1977] \\
\hline $\begin{array}{l}\mathrm{NH}_{3}+\mathrm{e} \rightarrow \\
\mathrm{NH}^{+}+\mathrm{H}_{2}+2 \mathrm{e}\end{array}$ & $1.71 \mathrm{E}-13$ & $9.21 \mathrm{E}-12$ & Mark et al. [1977] \\
\hline $\begin{array}{l}\mathrm{NH}_{3}+\mathrm{e}^{\mathrm{a}} \rightarrow \overrightarrow{\mathrm{H}} \\
\mathrm{NH}^{+}+\mathrm{H}_{2}+2 \mathrm{e}\end{array}$ & $1.49 \mathrm{E}-9$ & $1.52 \mathrm{E}-9$ & Mark et al. [1977] \\
\hline $\begin{array}{l}\mathrm{NH}_{3}+\mathrm{e} \rightarrow \\
\quad \mathrm{N}^{+}+\mathrm{H}_{2}+\mathrm{H}+2 \mathrm{e}\end{array}$ & $1.33 \mathrm{E}-14$ & $1.26 \mathrm{E}-12$ & Mark et al. [1977] \\
\hline $\begin{array}{l}\mathrm{NH}_{3}+\mathrm{e}^{\mathrm{a}} \rightarrow \\
\mathrm{N}^{+}+\mathrm{H}_{2}+\mathrm{H}+2 \mathrm{e}\end{array}$ & $5.44 \mathrm{E}-10$ & $5.67 \mathrm{E}-10$ & Mark et al. [1977] \\
\hline
\end{tabular}

${ }^{\mathrm{a}}$ Hot Electron Component. 
Table 3. Photoionization Rates

\begin{tabular}{|c|c|c|}
\hline Reaction & Enceladus-Dione & References \\
\hline $\mathrm{H}+\mathrm{h} \nu \rightarrow \mathrm{H}^{+}+\mathrm{e}$ & $8.0 \mathrm{E}-10$ & $\begin{array}{l}\text { Huebner and } \\
\text { Giguere [1980] }\end{array}$ \\
\hline $\mathrm{H}_{2}+\mathrm{h} \nu \rightarrow \mathrm{H}^{+}+\mathrm{H}+\mathrm{e}$ & $1.0 \mathrm{E}-10$ & $\begin{array}{l}\text { Huebner and } \\
\text { Giguere [1980] }\end{array}$ \\
\hline $\mathrm{H}_{2} \mathrm{O}+\mathrm{h} \nu \rightarrow \mathrm{H}^{+}+\mathrm{OH}+\mathrm{e}$ & $1.4 \mathrm{E}-10$ & $\begin{array}{l}\text { Huebner and } \\
\quad \text { Giguere [1980] }\end{array}$ \\
\hline $\mathrm{NH}_{3}+\mathrm{h} \nu \rightarrow \mathrm{H}^{+}+\mathrm{NH}_{2}+\mathrm{e}$ & 3.7E-11 & Huebner et al. [1992] \\
\hline $\mathrm{H}_{2}+\mathrm{h} \nu \rightarrow \mathrm{H}_{2}^{+}+\mathrm{e}$ & & $\begin{array}{l}\text { Huebner and } \\
\text { Giguere [1980] }\end{array}$ \\
\hline $\mathrm{H}_{2} \mathrm{O}+\mathrm{h} \nu \rightarrow \mathrm{H}_{2} \mathrm{O}^{+}+\mathrm{e}$ & $3.7 \mathrm{E}-9$ & $\begin{array}{l}\text { Huebner and } \\
\text { Giguere [1980] }\end{array}$ \\
\hline $\mathrm{O}+\mathrm{h} \nu \rightarrow \mathrm{O}^{+}+\mathrm{e}$ & $2.3 \mathrm{E}-9$ & $\begin{array}{l}\text { Huebner and } \\
\text { Giguere [1980] }\end{array}$ \\
\hline $\mathrm{H}_{2} \mathrm{O}+\mathrm{h} \nu \rightarrow \mathrm{O}^{+}+\mathrm{H}_{2}+\mathrm{e}$ & $6.4 \mathrm{E}-11$ & $\begin{array}{l}\text { Huebner and } \\
\text { Giguere [1980] }\end{array}$ \\
\hline $\mathrm{O}_{2}+\mathrm{h} \nu \rightarrow \mathrm{O}^{+}+\mathrm{O}+\mathrm{e}$ & $5.8 \mathrm{E}-10$ & $\begin{array}{l}\text { Huebner and } \\
\quad \text { Giguere [1980] }\end{array}$ \\
\hline $\mathrm{CO}_{2}+\mathrm{h} \nu \rightarrow \mathrm{O}^{+}+\mathrm{CO}+\mathrm{e}$ & $2.8 \mathrm{E}-10$ & Huebner et al. [1992] \\
\hline $\mathrm{O}_{2}+\mathrm{h} \nu \rightarrow \mathrm{O}_{2}^{+}+\mathrm{e}$ & $5.6 \mathrm{E}-9$ & $\begin{array}{l}\text { Huebner and } \\
\text { Giguere [1980] }\end{array}$ \\
\hline $\mathrm{OH}+\mathrm{h} \nu \rightarrow \mathrm{OH}^{+}+\mathrm{e}$ & $3.7 \mathrm{E}-9$ & $\begin{array}{l}\text { Huebner and } \\
\quad \text { Giguere [1980] }\end{array}$ \\
\hline $\mathrm{CO}_{2}+\mathrm{h} \nu \rightarrow \mathrm{CO}_{2}^{+}+\mathrm{e}$ & $2.6 \mathrm{E}-9$ & Huebner et al. [1992] \\
\hline $\mathrm{CO}_{2}+\mathrm{h} \nu \rightarrow \mathrm{CO}^{+}+\mathrm{O}+\mathrm{e}$ & $1.68 \mathrm{E}-10$ & Huebner et al. [1992] \\
\hline $\mathrm{CO}_{2}+\mathrm{h} \nu \rightarrow \mathrm{C}^{+}+\mathrm{O}_{2}+\mathrm{e}$ & $1.2 \mathrm{E}-10$ & Huebner et al. [1992] \\
\hline $\mathrm{NH}_{3}+\mathrm{h} \nu \rightarrow \mathrm{NH}_{3}^{+}+\mathrm{e}$ & $6.8 \mathrm{E}-9$ & Huebner et al. [1992] \\
\hline $\mathrm{NH}_{3}+\mathrm{h} \nu \rightarrow \mathrm{NH}_{2}^{+}+\mathrm{H}+\mathrm{z}$ & $1.96 \mathrm{E}-9$ & Huebner et al. [1992] \\
\hline $\mathrm{NH}_{3}+\mathrm{h} \nu \rightarrow \mathrm{NH}^{+}+\mathrm{H}_{2}+\mathrm{e}$ & $7.66 \mathrm{E}-11$ & Huebner et al. [1992] \\
\hline $\mathrm{NH}_{3}+\mathrm{h} \nu \rightarrow \mathrm{N}^{+}+\mathrm{H}_{2}+\mathrm{H}+\mathrm{e}$ & $3.6 \mathrm{E}-11$ & Huebner et al. [1992] \\
\hline
\end{tabular}

given above. Referring to Figure $4 \mathrm{a}$ for Dione, the $\mathrm{H}_{2} \mathrm{O}^{+}$ densities reach peak values of about 1.0 ions $/ \mathrm{cm}^{3}$ near the surface. $\mathrm{H}_{2}^{+}$has the lowest density, while $\mathrm{H}^{+}$and $\mathrm{O}_{2}^{+}$have peak densities of about 0.06 ions $/ \mathrm{cm}^{3}$ and 0.08 ions $/ \mathrm{cm}^{3}$, respectively. Pickup $\mathrm{O}^{+}$is relatively low in abundance relative to ambient densities and will probably be difficult to detect since the dominant ambient ion is expected to be $\mathrm{O}^{+}$. The relatively flat profile for $\mathrm{O}^{+}$at all heights, except

Table 4. Steady State Densities and Steady State Ion Electron Temperatures of Enceladus Torus and Dione Torus

\begin{tabular}{|c|c|c|}
\hline & Enceladus Torus & Dione Torus \\
\hline \multicolumn{3}{|c|}{ Steady State Densities, $\mathrm{cm}^{-3}$} \\
\hline $\mathrm{N}_{\mathrm{ce}}$ & 100.0 & 25.4 \\
\hline $\mathrm{N}_{\text {he }}$ & 0.2 & 0.4 \\
\hline $\mathrm{H}$ & 50 & 80 \\
\hline $\mathrm{H}^{+}$ & 25 & 4.6 \\
\hline $\mathrm{H}_{2}^{+}$ & $0.265^{\mathrm{a}}$ & $0.81^{\mathrm{a}}$ \\
\hline $\mathrm{O}$ & 100 & 180 \\
\hline $\mathrm{O}^{+}$ & 50 & 11.8 \\
\hline $\mathrm{OH}$ & 500 & 250 \\
\hline $\mathrm{OH}^{+}$ & 10 & 4.45 \\
\hline $\mathrm{H}_{2} \mathrm{O}$ & 100 & 30 \\
\hline $\mathrm{H}_{2} \mathrm{O}^{+}$ & 15 & 3.6 \\
\hline $\mathrm{O}_{2}$ & 0.0 & 0.0 \\
\hline $\mathrm{O}_{2}^{+}$ & $0.03^{\mathrm{a}}$ & $0.15^{\mathrm{a}}$ \\
\hline \multicolumn{3}{|c|}{ Steady State Ion Electron Temperatures, eV } \\
\hline $\mathrm{T}_{\mathrm{ce}}$ & 3.0 & 5.5 \\
\hline $\mathrm{T}_{\text {he }}$ & 100 & 145 \\
\hline $\mathrm{T}\left(\mathrm{H}^{+}\right)$ & 11 & 16.5 \\
\hline $\mathrm{T}\left(\mathrm{H}_{2}{ }^{+}\right)^{\mathrm{a}}$ & 22 & 33 \\
\hline $\mathrm{T}\left(\mathrm{O}^{+}\right)$ & 80 & 105 \\
\hline $\mathrm{T}\left(\mathrm{OH}^{+}\right)^{\mathrm{b}}$ & 85 & 111 \\
\hline $\mathrm{T}\left(\mathrm{H}_{2} \mathrm{O}^{+}\right)^{\mathrm{b}}$ & 90 & 118 \\
\hline $\mathrm{T}\left(\mathrm{O}_{2}^{+}\right)^{\mathrm{b}}$ & 160 & 210 \\
\hline
\end{tabular}

${ }^{\mathrm{a}}$ Richardson et al. [1986].

${ }^{\mathrm{b}}$ Assume temperature proportional to ion mass. near the surface, indicates we are primarily seeing pickup ions produced by ambient neutral oxygen. Figure $4 \mathrm{~b}$ shows pickup ion densities at Dione for $\mathrm{OH}^{+}, \mathrm{CO}_{2}^{+}, \mathrm{NH}_{3}^{+}, \mathrm{H}_{3} \mathrm{O}^{+}$, and $\mathrm{NH}_{2}^{+}$. Within $560 \mathrm{~km}$ in height all ions should be detectable. $\mathrm{OH}^{+}$dominates relative to the other ions in this figure and surprisingly, $\mathrm{H}_{3} \mathrm{O}^{+}$is relatively abundant. Near Dione's surface the total pickup ion density is about 1.4 ions $/ \mathrm{cm}^{3}$, which is about $5.6 \%$ the ambient density and we may expect to see a modest interaction at Dione. Here we note that because of the detection of ozone, $\mathrm{O}_{3}$, at Dione [Noll et al., 1997] we may see significant fluxes of pickup $\mathrm{O}_{2}^{+}$and $\mathrm{O}_{3}^{+}$within a few hundred kms above Dione's surface only for a wake pass. Therefore a strong interaction may occur similar to that observed by Voyager 1 at Titan [Neubauer et al., 1984].

[23] In the case of Enceladus, $\mathrm{H}_{2} \mathrm{O}^{+}$clearly dominates over all other ions with peak densities near the surface of 2.2 ions $/ \mathrm{cm}^{3}$. The next most important ion is $\mathrm{O}_{2}^{+}$with peak densities near 0.15 ions $/ \mathrm{cm}^{3}$ at the surface. The other ions $\mathrm{H}^{+}, \mathrm{O}^{+}$, and $\mathrm{H}_{2}^{+}$are of low abundance and will only be detectable close to the surface. Again, because of the high
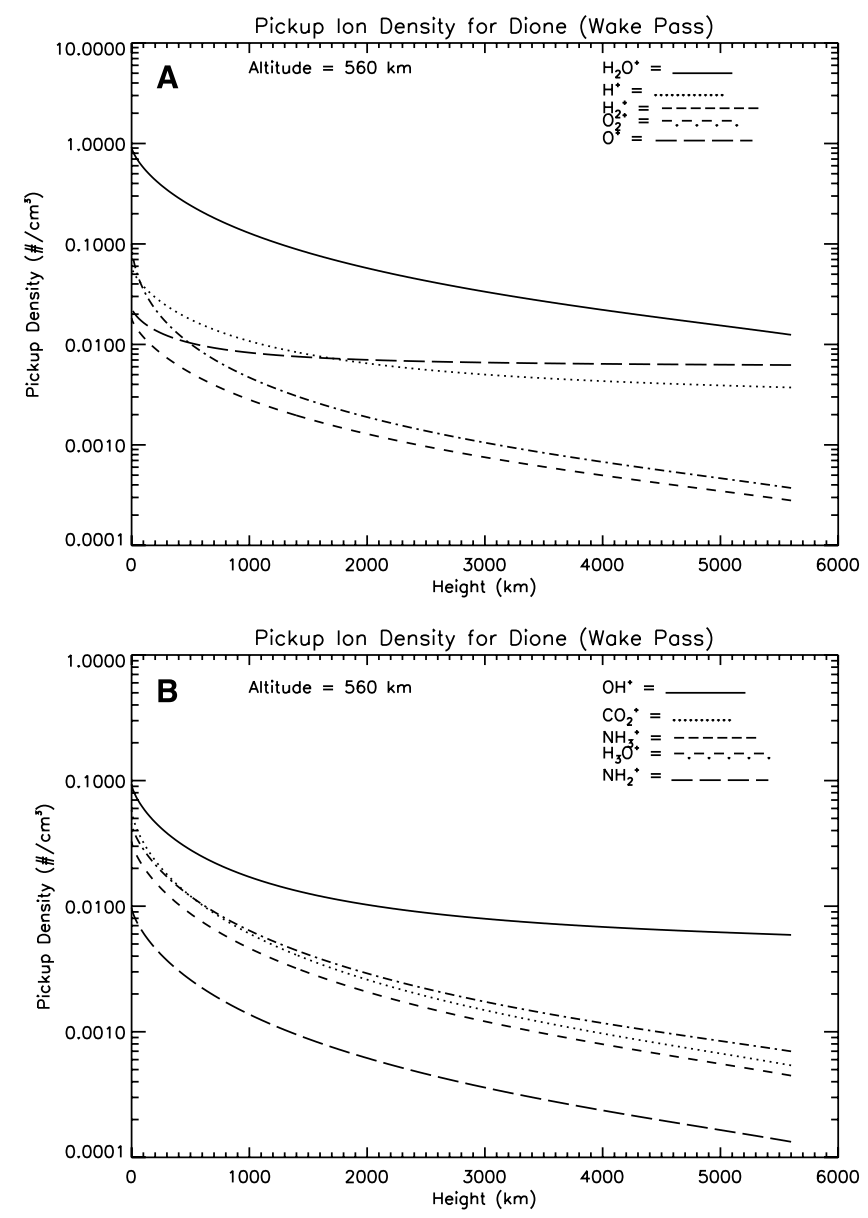

Figure 4. (a) Computed ion densities at spacecraft for Dione wake flyby as a function of height for $\mathrm{H}_{2} \mathrm{O}^{+}, \mathrm{O}_{2}^{+}, \mathrm{H}_{2}^{+}$, $\mathrm{O}^{+}$, and $\mathrm{H}^{+}$ions. The height is equal to the $\alpha-\mathrm{r}_{0}$ parameter in Figure 3. (b) Computed ion densities at spacecraft for Dione wake flyby as a function of height for $\mathrm{OH}^{+}, \mathrm{CO}_{2}^{+}$, $\mathrm{NH}_{3}^{+}, \mathrm{NH}_{2}^{+}$, and $\mathrm{H}_{3} \mathrm{O}^{+}$. The height is equal to the $\alpha-\mathrm{r}_{0}$ parameter in Figure 3. 

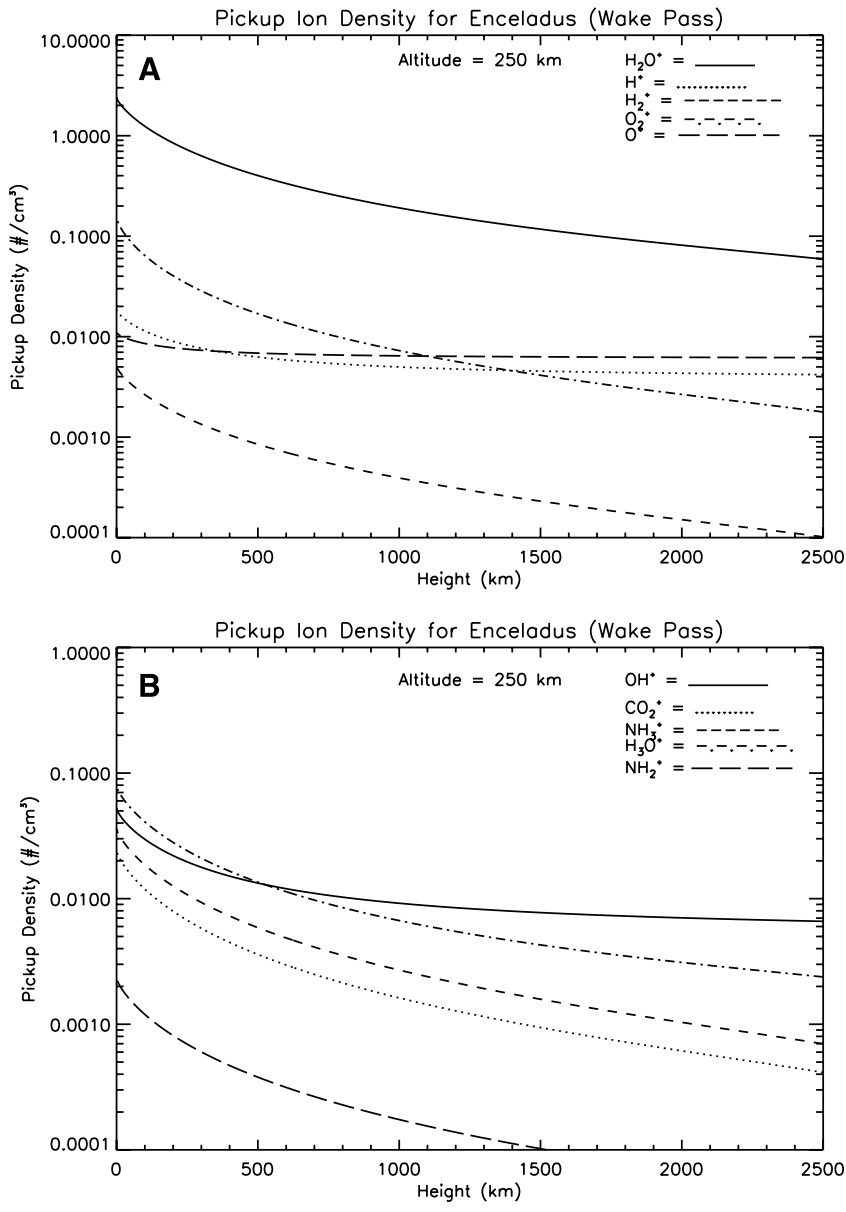

Figure 5. (a) Computed ion densities at spacecraft for Enceladus wake flyby as a function of height for $\mathrm{H}_{2} \mathrm{O}^{+}, \mathrm{O}_{2}^{+}$, $\mathrm{H}_{2}^{+}, \mathrm{O}^{+}$, and $\mathrm{H}^{+}$ions. The height is equal to the $\alpha-\mathrm{r}_{0}$ parameter in Figure 3. (b) Computed ion densities at spacecraft for Enceladus wake flyby as a function of height for $\mathrm{OH}^{+}, \mathrm{CO}_{2}^{+}, \mathrm{NH}_{3}^{+}, \mathrm{NH}_{2}^{+}$, and $\mathrm{H}_{3} \mathrm{O}^{+}$. The height is equal to the $\alpha-r_{0}$ parameter in Figure 3.

abundance of ambient $\mathrm{H}^{+}$and $\mathrm{O}^{+}$, pickup $\mathrm{H}^{+}$and $\mathrm{O}^{+}$will be undetectable. In Figure $5 \mathrm{~b}$ the ions $\mathrm{H}_{3} \mathrm{O}^{+}, \mathrm{OH}^{+}, \mathrm{CO}_{2}^{+}$, and $\mathrm{NH}_{3}^{+}$should all be detectable. The ion $\mathrm{NH}_{2}^{+}$may be detectable close to Enceladus' surface. Here we are using as a rule of thumb for CAPS ability of detection of 0.001 ions $/ \mathrm{cm}^{3}$ for ST data and 0.01 ions $/ \mathrm{cm}^{3}$ for LEF data. This will become more apparent when we show our ring distribution simulations. Figures 4 and 5 also show the height variation of the pickup ion densities, and as mentioned previously, this height variation will give information on the energy distribution of the sputtered neutrals.

\subsection{Upstream Passes}

[24] In Figures 6a and 6b we show the pickup ion density as a function of distance $\mathrm{Y}$ for an upstream pass with closest approach altitude of $500 \mathrm{~km}$ for Dione. Y is the distance along the spacecraft trajectory relative to the center of the body (i.e., $\mathrm{Y}=0$ corresponds to closest approach). In the case of $\mathrm{H}_{2} \mathrm{O}^{+}$we have detectable pickup ion fluxes for the ion measurements by CAPS. The $\mathrm{H}_{2}^{+}$and $\mathrm{O}_{2}^{+}$measurements are also possible for pickup ions, since we can use the ST measurements for them. $\mathrm{H}^{+}$and $\mathrm{O}^{+}$pickup ions will be undetectable because of the relatively high ambient densities of these species. Referring to Figure $6 \mathrm{~b}$, we can probably rule out the detection of $\mathrm{H}_{3} \mathrm{O}^{+}$using LEF data because of oxygen's high affinity for electrons. $\mathrm{CO}_{2}^{+}$(ST detection) pickup ion detection is clearly possible, since it is well separated from the water group ions. $\mathrm{NH}_{3}^{+}$detection using LEF may be ruled out because of its low abundance. It will be difficult to use ST data for $\mathrm{OH}^{+}$and $\mathrm{H}_{3} \mathrm{O}^{+}$ detection, since ambient $\mathrm{O}^{+}$and $\mathrm{H}_{2} \mathrm{O}^{+}$will dominate. Mapping the ring distributions will greatly increase our chances for detecting pickup ions such as $\mathrm{NH}_{3}^{+}, \mathrm{OH}^{+}$, and $\mathrm{H}_{3} \mathrm{O}^{+}$relative to the ambient ions. We still need to separate the pickup $\mathrm{NH}_{3}^{+}, \mathrm{OH}^{+}$, and $\mathrm{H}_{3} \mathrm{O}^{+}$from pickup $\mathrm{H}_{2} \mathrm{O}^{+}$, which has similar M/Q. In our model pickup $\mathrm{NH}_{3}^{+}$is $3 \%$ of pickup $\mathrm{H}_{2} \mathrm{O}^{+}$but $\mathrm{NH}_{3}^{+}$may be enriched in the LEF spectra because oxygen has a high electron affinity. In the case of ST data, $\mathrm{NH}_{3}^{+}$cannot be separated from $\mathrm{OH}^{+}$since they have the same $\mathrm{M} / \mathrm{Q}$.

[25] In Figures 7a and 7b we show the pickup ion density as a function of distance $\mathrm{Y}$ for an upstream pass with closest
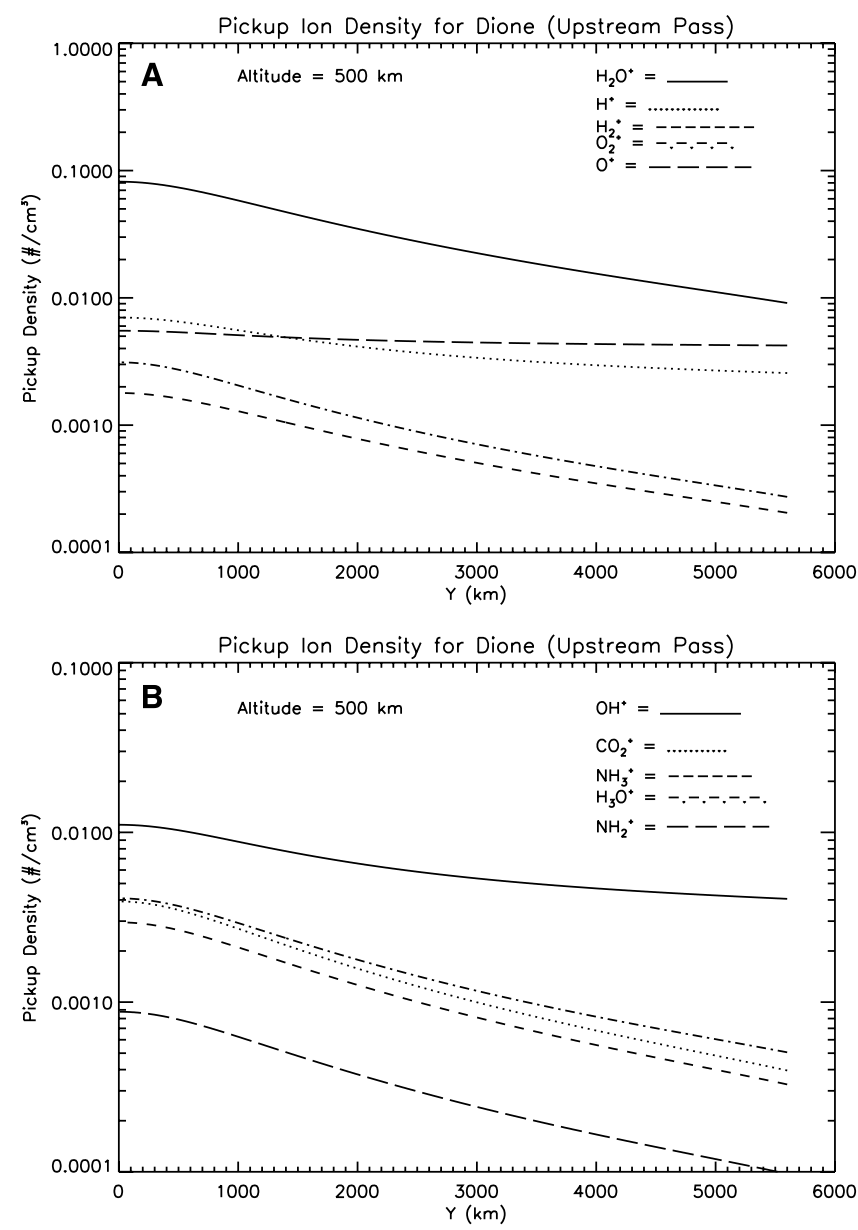

Figure 6. (a) Computed ion densities at spacecraft for Dione upstream pass as a function of transverse distance $\mathrm{Y}=\alpha$ (see Figure 3) with $500 \mathrm{~km}$ closest approach altitude for $\mathrm{H}_{2} \mathrm{O}^{+}, \mathrm{O}_{2}^{+}, \mathrm{H}_{2}^{+}, \mathrm{O}^{+}$, and $\mathrm{H}^{+}$ions. (b) Computed ion densities at spacecraft for Dione upstream pass as a function of transverse distance $\mathrm{Y}=\alpha$ (see Figure 3) with $500 \mathrm{~km}$ closest approach altitude for $\mathrm{OH}^{+}, \mathrm{CO}_{2}^{+}, \mathrm{NH}_{3}^{+}, \mathrm{NH}_{2}^{+}$, and $\mathrm{H}_{3} \mathrm{O}^{+}$ions. 

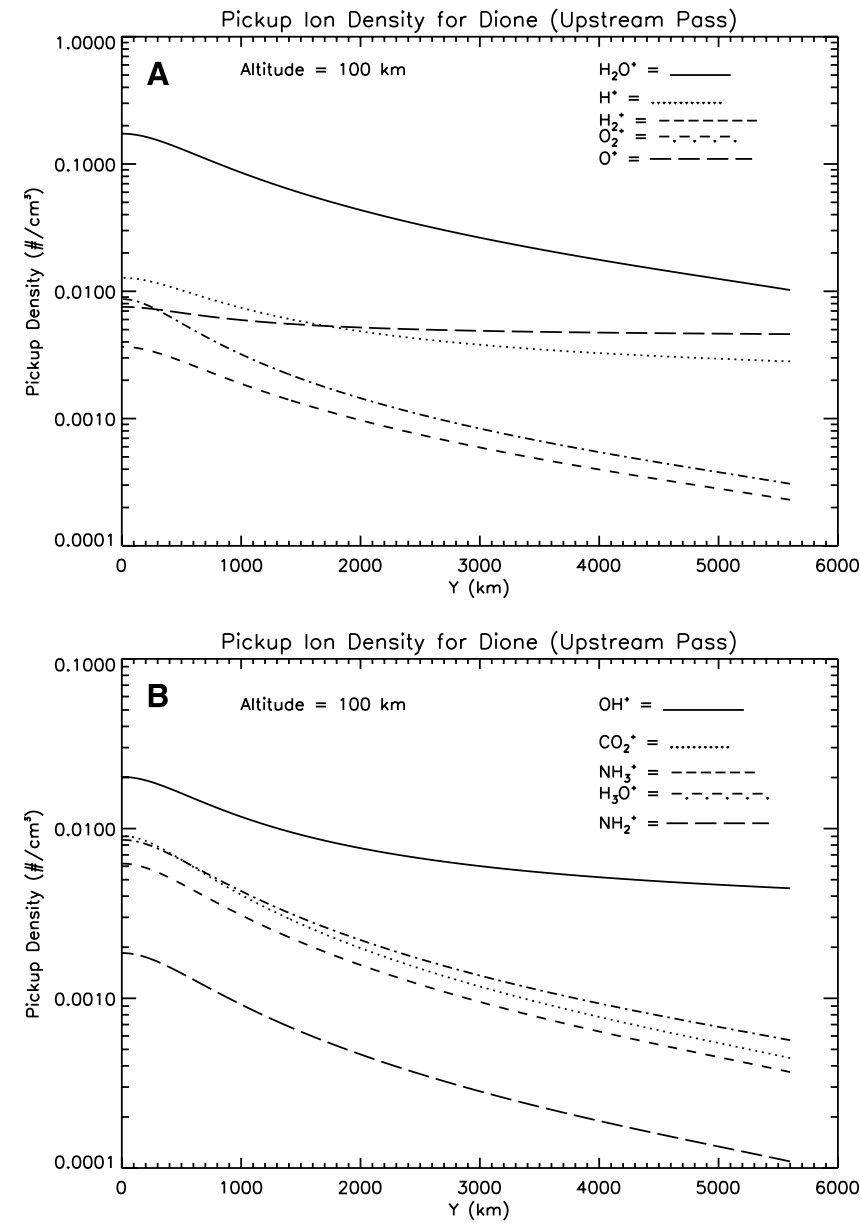

Figure 7. (a) Computed ion densities at spacecraft for Dione upstream pass as a function of transverse distance $\mathrm{Y}=\alpha$ (see Figure 3) with $100 \mathrm{~km}$ closest approach altitude for $\mathrm{H}_{2} \mathrm{O}^{+}, \mathrm{O}_{2}^{+}, \mathrm{H}_{2}^{+}, \mathrm{O}^{+}$, and $\mathrm{H}^{+}$ions. (b) Computed ion densities at spacecraft for Dione upstream pass as a function of transverse distance $\mathrm{Y}=\alpha$ (see Figure 3) with $100 \mathrm{~km}$ closest approach altitude for $\mathrm{OH}^{+}, \mathrm{CO}_{2}^{+}, \mathrm{NH}_{3}^{+}, \mathrm{NH}_{2}^{+}$, and $\mathrm{H}_{3} \mathrm{O}^{+}$ions.

approach altitude of $100 \mathrm{~km}$ for Dione. For navigation reasons, such a close encounter is not allowed but is shown to compare upstream pass versus wake pass. Here, ST detection of $\mathrm{H}_{2} \mathrm{O}^{+}, \mathrm{O}_{2}^{+}$, and $\mathrm{H}_{2}^{+}$pickup ions is possible out to several thousand kilometers. $\mathrm{H}^{+}$and $\mathrm{O}^{+}$pickup ions are again not detectable because of high ambient densities for these ion species. In Figure $7 \mathrm{~b}$, ions, $\mathrm{OH}^{+}$(LEF detection), and $\mathrm{CO}_{2}^{+}$(ST detection) are possible out to $1000 \mathrm{~km}$. To detect $\mathrm{NH}_{3}^{+}$and $\mathrm{H}_{3} \mathrm{O}^{+}$, we would need LEF detection requiring peak densities in excess of 0.01 ions $/ \mathrm{cm}^{3}$. Because these ions form ring distributions, their detection relative to ambient ions may be possible. For ST data we cannot separate $\mathrm{NH}_{3}^{+}$and $\mathrm{OH}^{+}$, since they have the same $\mathrm{M} / \mathrm{Q}$. $\mathrm{NH}_{2}^{+}$(LEF detection) pickup ion detection is also not possible. Since we are assuming 10\% abundance, direct detection of pickup ions for many of the minor species might not be possible if they are present at the few percent level. However, such ions can be detected as they accumulate in the ambient plasma. Finally, the use of B cycle data, with its high sensitivity, may allow detection of minor ions at the few percent level, although we could not uniquely identify them as fresh ions which would have a ring distribution in velocity space.

[26] In Figures 8a and 8b we show the pickup ion density as a function of distance $Y$ for an upstream pass with closest approach altitude of $500 \mathrm{~km}$ for Enceladus. In Figure 8a, ST detection is possible at all altitudes for $\mathrm{H}_{2} \mathrm{O}^{+}$and $\mathrm{O}_{2}^{+}$but not $\mathrm{H}_{2}^{+} \cdot \mathrm{H}^{+}$and $\mathrm{O}^{+}$are again not detectable because of the high ambient densities of these species. In Figure 8b, of $\mathrm{OH}^{+}$, $\mathrm{H}_{3} \mathrm{O}^{+}$and $\mathrm{NH}_{3}^{+}$pickup ions are below the detection threshold of the LEF feature of the instrument. Although they compete with ambient $\mathrm{O}^{+}$and $\mathrm{H}_{2} \mathrm{O}^{+}$, detection of $\mathrm{OH}^{+}$and $\mathrm{H}_{3} \mathrm{O}^{+}$may be possible because of their ring distributions. In the ST data, $\mathrm{CO}_{2}^{+}$is barely detectable and $\mathrm{NH}_{2}^{+}$is not detectable.

[27] In Figures 9a and $9 \mathrm{~b}$ we show the pickup ion density as a function of distance $\mathrm{Y}$ for an upstream pass with closest approach altitude of $100 \mathrm{~km}$ for Enceladus,
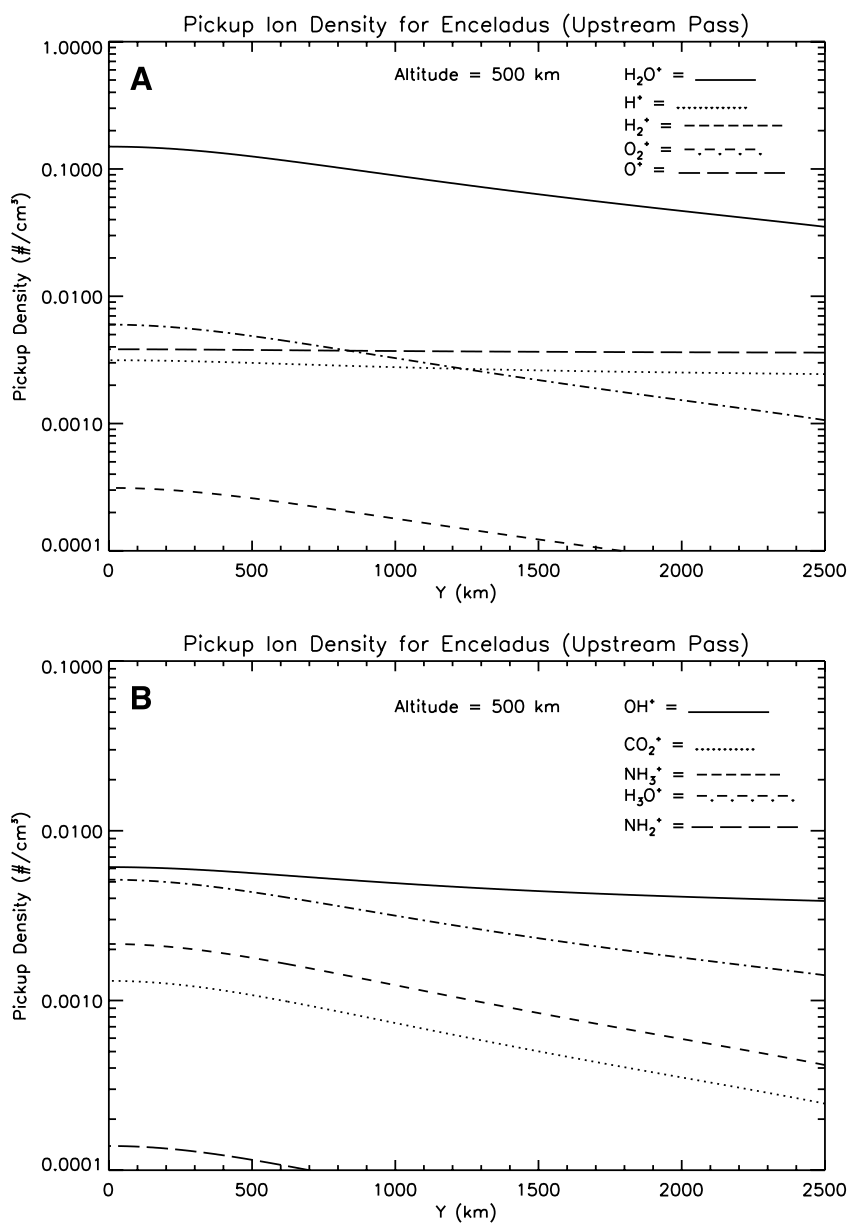

Figure 8. (a) Computed ion densities at spacecraft for Enceladus upstream pass as a function of transverse distance $\mathrm{Y}=\alpha$ (see Figure 3) with $500 \mathrm{~km}$ closest approach altitude for $\mathrm{H}_{2} \mathrm{O}^{+}, \mathrm{O}_{2}^{+}, \mathrm{H}_{2}^{+}, \mathrm{O}^{+}$, and $\mathrm{H}^{+}$ions. (b) Computed ion densities at spacecraft for Enceladus upstream pass as a function of transverse distance $\mathrm{Y}=\alpha$ (see Figure 3) with $500 \mathrm{~km}$ closest approach altitude for $\mathrm{OH}^{+}, \mathrm{CO}_{2}^{+}, \mathrm{NH}_{3}^{+}$, $\mathrm{NH}_{2}^{+}$, and $\mathrm{H}_{3} \mathrm{O}^{+}$ions. 

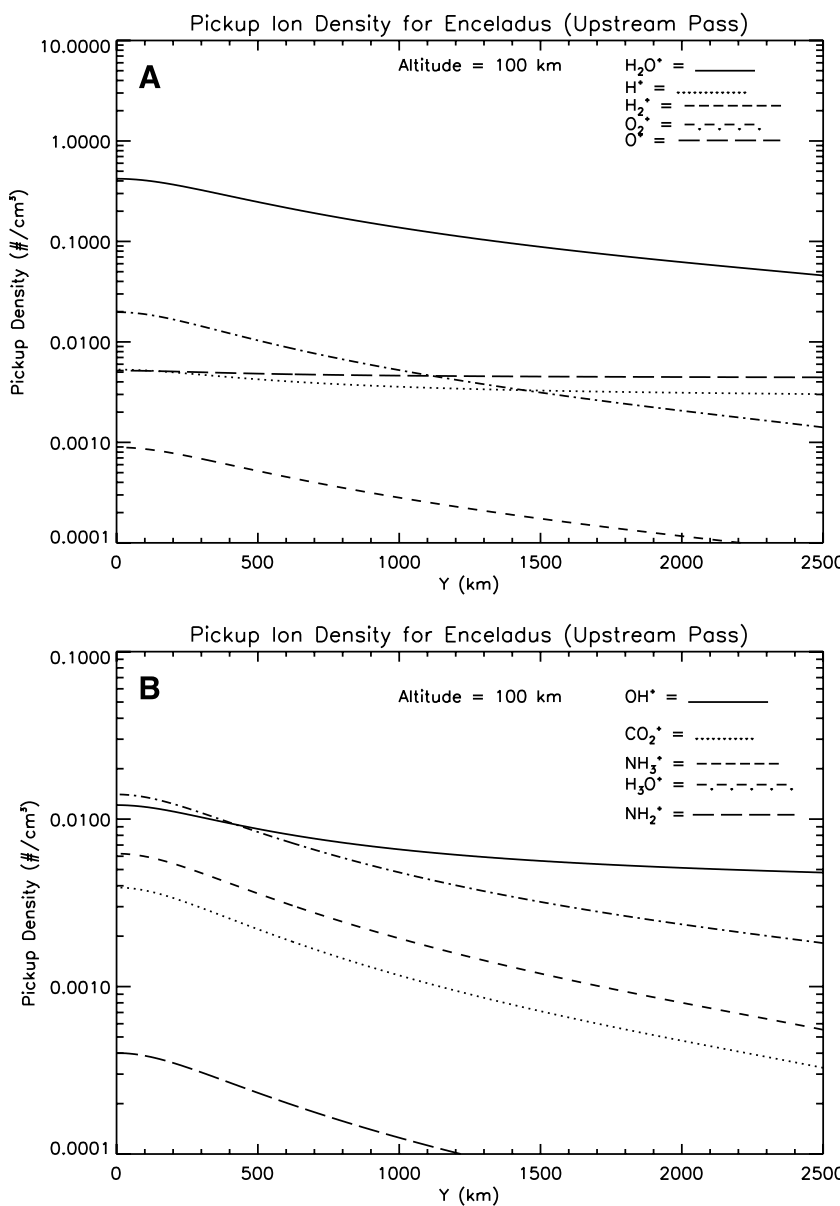

Figure 9. (a) Computed ion densities at spacecraft for Enceladus upstream pass as a function of transverse distance $\mathrm{Y}=\alpha$ (see Figure 3) with $100 \mathrm{~km}$ closest approach altitude for $\mathrm{H}_{2} \mathrm{O}^{+}, \mathrm{O}_{2}^{+}, \mathrm{H}_{2}^{+}, \mathrm{O}^{+}$, and $\mathrm{H}^{+}$ions. (b) Computed ion densities at spacecraft for Enceladus upstream pass as a function of transverse distance $\mathrm{Y}=\alpha$ (see Figure 3) with $100 \mathrm{~km}$ closest approach altitude for $\mathrm{OH}^{+}, \mathrm{CO}_{2}^{+}, \mathrm{NH}_{3}^{+}$, $\mathrm{NH}_{2}^{+}$, and $\mathrm{H}_{3} \mathrm{O}^{+}$ions.

again a trajectory not allowed by navigation but shown for comparing upstream and wake passes. In Figure 9a, ST detection of $\mathrm{H}_{2} \mathrm{O}^{+}$and $\mathrm{O}_{2}^{+}$pickup ions is possible at all distances, but $\mathrm{H}_{2}^{+}$detection is not possible. The detection of $\mathrm{H}^{+}$and $\mathrm{O}^{+}$pickup ions is again not possible because of the large ambient ion densities for these species. In Figure 9b, LEF detection of $\mathrm{OH}^{+}$and $\mathrm{H}_{3} \mathrm{O}^{+}$pickup ions may be possible inside $1000 \mathrm{~km}$. As above, they may be seen in the ST data at all heights, but then they must compete with ambient $\mathrm{O}^{+}, \mathrm{OH}^{+}$, and $\mathrm{H}_{2} \mathrm{O}^{+}$. LEF detection of $\mathrm{NH}_{3}^{+}$pickup ions may not possible at all heights, but because they form ring distributions and $\mathrm{NH}_{3}^{+}$is enriched relative to water group ions (i.e., high electron affinity of oxygen) they maybe detectable. $\mathrm{CO}_{2}^{+}$(ST detection) pickup ion detection is possible at all heights; $\mathrm{NH}_{2}^{+}$(ST or LEF) pickup ion detection is not possible at all heights.

[28] At this point we would like to add the following caveats: many of the minor ions for which detection using either ST or LEF data was ruled out was based on the ion count measurements or A cycle data. With good B cycle data, which is considerably more sensitive, we may be able to detect these minor ions. However, such detection would provide minimal information about the pitch angle distributions of the various ion species, which is needed to distinguish between ambient ions and pickup ions. However, if we see a significant rise in signal in the B-cycle data as the spacecraft approaches the satellite, this would indicate the presence of freshly picked up ions.

\section{Ring Distribution Simulations}

[29] Here we derive the expressions used to compute the simulated pickup ion count rates for CAPS. These calculations assume that the ions are in the form of a ring distribution and have not had sufficient time to pitch angle scatter before they are detected by CAPS [see $W u$ and Davison, 1972; Hartle and $W u, 1973]$. In the plasma frame of reference one can write the velocity distribution for a fresh pickup ion [Hartle and $W u, 1973$ ]

$$
f\left(\vec{v}^{*}\right)=\frac{n}{2 \pi v_{0 \perp}} \delta\left(\vec{v}_{\perp}^{*}-\vec{v}_{0 \perp}\right) \delta\left(v_{z}^{*}-v_{0 z}\right),
$$

where $\vec{v}^{*}$ is the particle velocity in the plasma frame, with $\vec{v}_{0}$ the mean velocity of the plasma which will be roughly in the direction of corotation. The component perpendicular to the magnetic field is indicated by the symbol $\perp$ and the component along the magnetic field is along the $\mathrm{z}$ axis. One can then rewrite the above expression in the appropriate form when viewed in the spacecraft frame ignoring for now spacecraft motion

$f(\vec{v})=\frac{n}{4 V_{x}} \delta\left(v_{z}\right)\left[\delta\left(v_{y}-\sqrt{2 v_{x} V_{x}-v_{x}^{2}}\right)+\delta\left(v_{y}+\sqrt{2 v_{x} V_{x}-v_{x}^{2}}\right)\right]$

Here $\mathrm{V}_{\mathrm{x}}$ is the flow speed of the ambient ions with $\mathrm{x}$ along the corotation direction; $\vec{v}$ is the velocity of the pickup ion in the spacecraft frame. Finally, this expression can be further reformulated into a more useful form

$$
f(\vec{v})=\frac{n}{2 \pi \sqrt{2 v_{x} V_{x}}} \delta\left(v_{r}-\sqrt{2 v_{x} V_{x}}\right) \delta\left(v_{z}\right)
$$

In Figure 10 we show a schematic of the ring distribution in the spacecraft frame. We are now in a position to incorporate this expression in the equation defining the observed count rate for a given distribution function $f(\vec{v})$. The general expression for the count rate that CAPS would observe is

$$
C R=\varepsilon A \int_{\Delta v} \int_{\Delta \Omega} R(E / Q, \theta, \phi ; \vec{v}) f(\vec{v}) \vec{v} \cdot \hat{n} v_{r} d v_{r} d v_{z} d \phi,
$$

for which $R(E / Q, \theta, \phi ; \vec{v})$ is the response function of the IMS with E/Q the tuned energy-per-charge of the IMS, $\theta$ is the angle relative to the collimator normal of the IMS, and $\phi$ is the angular sector of the IMS. This equation reduces to the following expression after substituting the above expression for $\mathrm{f}$

$$
C R=\frac{\varepsilon A n \sqrt{2 v_{x} V_{x}} \Delta \phi}{2 \pi} .
$$




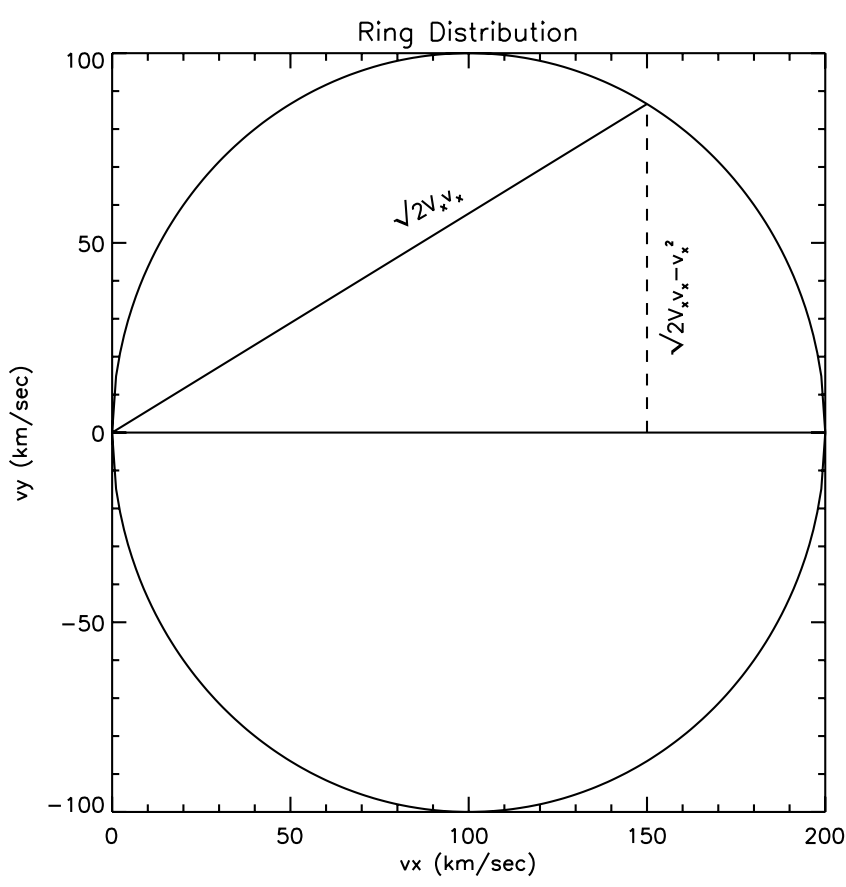

Figure 10. Ring velocity distribution for pickup ions with drift speed $\mathrm{V}_{\mathrm{x}}$.

Here $\varepsilon$ represents the detection efficiency of the IMS, A is its effective area of detection, and $\Delta \phi$ is the angular width of the IMS angular sectors which are $\sim 20^{\circ}$. We then introduce the geometric factor $\mathrm{G}=\varepsilon A \Delta \Omega \Delta E / E \approx 10^{-3} \mathrm{~cm}^{2}$ ster-eV/eV from which we get

$$
C R=\left(\frac{G}{\Delta \Omega \Delta E / E}\right)\left(\frac{n \Delta \phi}{2 \pi}\right)\left(\sqrt{2 v_{x} V_{x}}\right)
$$

for which $\Delta \Omega=0.05$ ster, $\Delta \mathrm{E} / \mathrm{E}=0.17$, and $0 \leq \mathrm{v}_{\mathrm{x}} \leq 2 \mathrm{~V}_{\mathrm{x}}$. In the limit of $\mathrm{v}_{\mathrm{x}}=2 \mathrm{~V}_{\mathrm{x}}$ and setting $\mathrm{V}_{\mathrm{x}} \approx 50 \mathrm{~km} / \mathrm{s}$, we get

$$
C R=1.2 \times 10^{5} n \quad \text { cps } .
$$

for ST data, where $\mathrm{n}$ is the density of the pickup ion in units of ions $/ \mathrm{cm}^{3}$. So, it can be seen that modest densities can produce very high count rates in CAPS if we sample the ring distribution function before it can pitch angle scatter and spread out in phase space.

[30] Simulations of the pickup ions plus ambient ions for various species are shown in Figure 11 for Dione and Figure 12 for Enceladus (i.e., A cycle data) for which we assumed for Dione pickup densities of 1.0 ions $/ \mathrm{cm}^{3}$ for $\mathrm{H}_{2} \mathrm{O}^{+}, 0.08$ ions $/ \mathrm{cm}^{3}$ for $\mathrm{O}_{2}^{+}, 0.06$ ions $/ \mathrm{cm}^{3}$ for $\mathrm{CO}_{2}^{+}$, and 0.03 ions $/ \mathrm{cm}^{3}$ for $\mathrm{NH}_{3}^{+}$and for Enceladus we assumed pickup densities of 2.2 ions $/ \mathrm{cm}^{3}$ for $\mathrm{H}_{2} \mathrm{O}^{+}, 0.15$ ions $/ \mathrm{cm}^{3}$ for $\mathrm{O}_{2}^{+}, 0.025$ ions $/ \mathrm{cm}^{3}$ for $\mathrm{CO}_{2}^{+}$, and 0.04 ions $/ \mathrm{cm}^{3}$ for $\mathrm{NH}_{3}^{+}$. These pickup ion densities were derived from our wake pass calculations at zero height. For the ambient ions we assumed convected Maxwellians and used the ion parameters in Table 4. For reference we show the energyangle coverage provided by CAPS in Figures $13 \mathrm{a}$ and $13 \mathrm{~b}$ for $\mathrm{O}_{2}^{+}$at Dione and Enceladus, respectively. It should be clear from Figures 11, 12, and 13 that in most cases CAPS will be able to measure the ring distributions of the pickup ions for such species as $\mathrm{H}_{2} \mathrm{O}^{+}, \mathrm{O}_{2}^{+}, \mathrm{CO}_{2}^{+}$, and $\mathrm{NH}_{3}^{+}$during a wake pass. This should also be true for some of the other ion species such as $\mathrm{OH}^{+}$and $\mathrm{H}_{3} \mathrm{O}^{+}$. The contour plots show that the count rates can exceed several $100 \mathrm{kHz}$ for the pickup ions and in many cases should allow us to see them many 1000 of kilometers above the satellites surface. Here, we note that for the molecule $\mathrm{NH}_{3}^{+}$we prefer to use LEF data, so the displayed counts in Figures $11 \mathrm{~d}$ and $12 \mathrm{~d}$ will be an order of magnitude lower for LEF data. Although one might still detect $\mathrm{NH}_{3}^{+}$in the $\mathrm{ST}$ data because they form ring distributions, we cannot distinguish them from $\mathrm{OH}^{+}$pickup ions which have the same M/Q. Therefore LEF data is preferred. Another reason why we prefer to use LEF data to detect $\mathrm{NH}_{3}^{+}$ions is that oxygen forms a weaker signal in LEF relative to nitrogen because its higher electron affinity. This is supported by calibration data.

\section{Simulated E/Q Versus TOF Spectrograms}

[31] We are now in a position to compute simulated $\mathrm{E} / \mathrm{Q}$ versus TOF contour plots of ion counts for both ST and LEF data (i.e., B cycle data) for a wake pass only. These simulations will require us to integrate along the spacecraft trajectory over distances $\sim 3000 \mathrm{~km}$. Therefore this data set will not provide information about the radial variation of the ion density or information about the pitch angle distributions. Here, we note that for scale sizes $>3000 \mathrm{~km}$ it will provide some information about the radial dependence of some pickup ion species relative to the targeted satellites. Considering the time and spatial scales of each encounter, we will get one spectrum before closest approach and one spectrum after closest approach. However, the advantage of this data set is that it provides the energy spectrum of each ion species detected and because of its higher sensitivity and observed TOF spectrum allows us to detect the minor ions more easily and detect unexpected ion species. The results of these simulations are shown in Figures $14 \mathrm{a}$ and $14 \mathrm{~b}$ for Dione and Figures $15 \mathrm{a}$ and $15 \mathrm{~b}$ for Enceladus. We display the data using the full energy range of the spectrometer where we have added nearby energy steps as done for the $\mathrm{B}$ cycle data (i.e., $32 \mathrm{E} / \mathrm{Q}$ steps). Note that the spectrograms are plotted versus TOF channel number for which each channel is about 0.75 ns wide. We have also added Poisson statistical noise to the data so that the simulations give a more realistic description of the data.

[32] Some caveats with regard to the ST simulations need to be said. First, for the $\mathrm{H}^{+}$and $\mathrm{H}_{2}^{+}$data we have ignored the importance of the $\mathrm{H}^{-}$fragment, which can exit the carbon foil; this fragment is relatively small when compared with the neutral $\mathrm{H}$ fragment. At present we do not have reliable $\mathrm{C}^{+}$calibration data and are using appropriately shifted $\mathrm{N}^{+}$calibration data for our $\mathrm{C}^{+}$ions; here we are ignoring the $\mathrm{C}^{-}$fragment for incident $\mathrm{C}^{+}$ions. For $\mathrm{NH}^{+}$we are also using appropriately shifted $\mathrm{N}^{+}$calibration data so that the $\mathrm{H}^{-}$fragment is being ignored. For the remainder of the species $\mathrm{O}^{+}$calibration data is predominately used and this calibration data was contaminated with methane, which was evidently in the calibration facilities ion source. So, this data had $\mathrm{C}^{-}$and $\mathrm{H}^{-}$fragments. In cases of $\mathrm{OH}^{+}, \mathrm{H}_{2} \mathrm{O}^{+}$and $\mathrm{H}_{3} \mathrm{O}^{+}$we kept the $\mathrm{H}^{-}$fragment but deleted the $\mathrm{C}^{-}$fragment. For $\mathrm{O}^{+}$and $\mathrm{O}_{2}^{+}$we deleted the $\mathrm{H}^{-}$and $\mathrm{C}^{-}$fragments. For $\mathrm{NH}_{3}^{+}$we deleted the $\mathrm{C}^{-}$and $\mathrm{O}^{-}$fragments, while for 

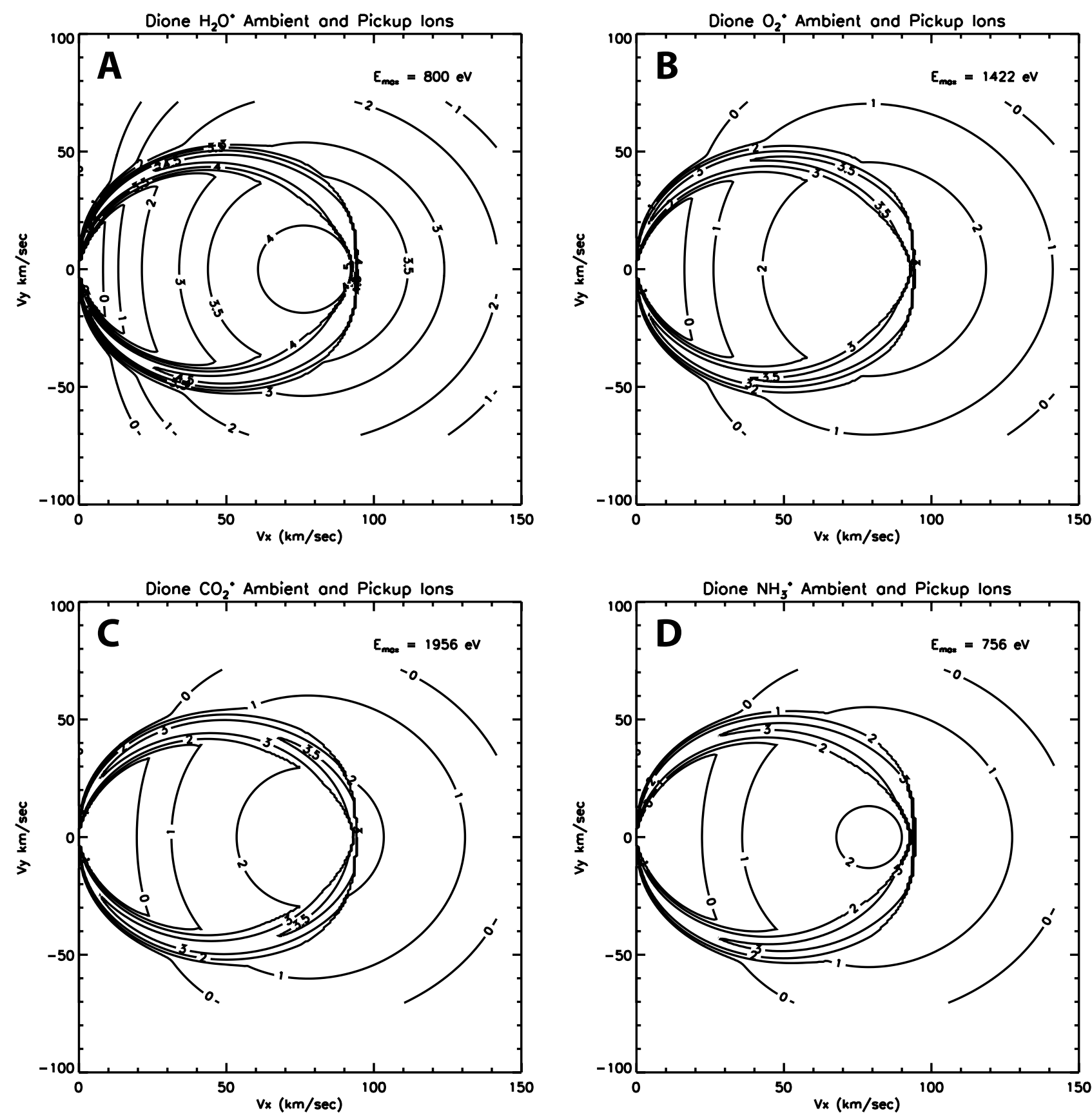

Figure 11. Contour plots of simulated ion counts at Dione for (a) $\mathrm{H}_{2} \mathrm{O}^{+}$, (b) $\mathrm{O}_{2}^{+}$, (c) $\mathrm{CO}_{2}^{+}$, and (d) $\mathrm{NH}_{3}^{+}$. The contour levels are $\log _{10}($ counts/s).

$\mathrm{CO}_{2}^{+}$and $\mathrm{CO}^{+}$we deleted the $\mathrm{H}^{-}$fragment. In the ST data there are also LEF ghost peaks. We have corrected the position of the $\mathrm{NH}^{+}$ghost peak relative to that for $\mathrm{N}^{+}$. The same can be said for the ghost peak positions $\mathrm{OH}^{+}, \mathrm{H}_{2} \mathrm{O}^{+}$, $\mathrm{H}_{3} \mathrm{O}^{+}$, and $\mathrm{NH}_{3}^{+}$relative to that for $\mathrm{O}^{+}$. We have also added the appropriate ghost peaks for $\mathrm{CO}_{2}^{+}, \mathrm{CO}^{+}$, and $\mathrm{O}_{2}^{+}$ which were derived from $\mathrm{S}^{+}$ion calibration data. In the case of $\mathrm{CO}_{2}^{+}$and $\mathrm{CO}^{+}$there are two ghost peaks, one for $\mathrm{C}^{+}$and the other for $\mathrm{O}^{+}$. Because of the detuning nature of the LEF for molecule species like $\mathrm{O}_{2}^{+}$where the exit energies of the fragments are $\ll 15 \mathrm{kV}$, the LEF ghost peak will be shifted to shorter TOF relative to that for incident atomic $\mathrm{O}^{+}$by a considerable amount and the ghost peak will be very broad in TOF. At present the relative abundance of methane and oxygen in the ion source is not known, so there is some intrinsic error in the relative heights of the different fragments for the various species. At this time these minor corrections do not impact the goals of this paper but are issues to be dealt with when it comes to the final calibration of the instrument (i.e., using prototype and engineering versions of flight unit) and operation of the instrument within Saturn's magnetosphere and surrounding solar wind.

[33] The simulations of the ST data in Figures 14a and $15 \mathrm{a}$ show that we can resolve the $\mathrm{H}^{+}$and $\mathrm{H}_{2}^{+}$in the $\mathrm{ST}$ data 

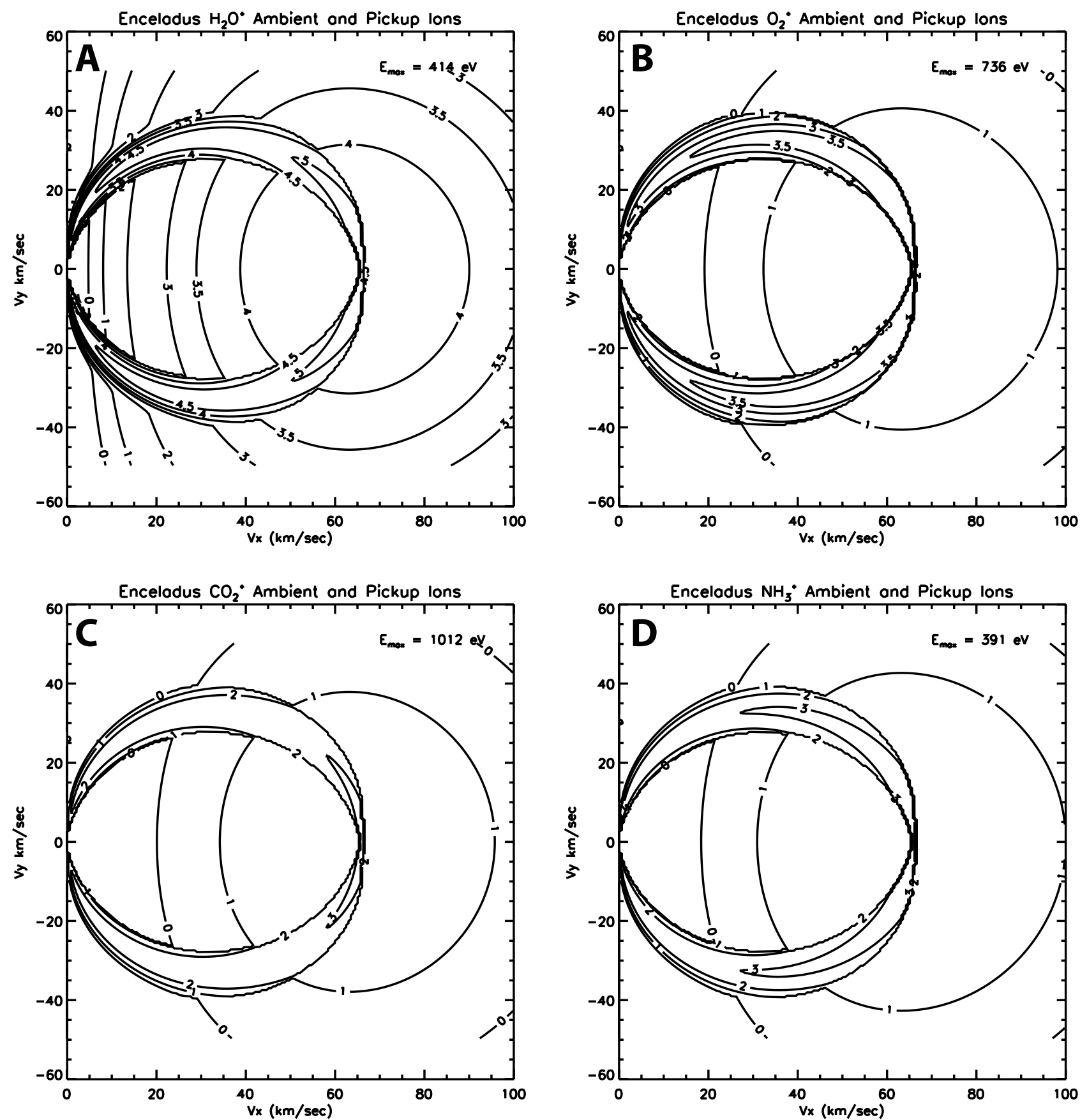

Figure 12. Contour plots of simulated ion counts at Enceladus for (a) $\mathrm{H}_{2} \mathrm{O}^{+}$, (b) $\mathrm{O}_{2}^{+}$, (c) $\mathrm{CO}_{2}^{+}$, and (d) $\mathrm{NH}_{3}^{+}$. The contour levels are $\log _{10}($ counts/s).

for which the $\mathrm{H}_{2}^{+}$extend to higher energy because of its greater mass. The same can be said for the water group ions being separated from both $\mathrm{O}_{2}^{+}$(i.e., note that in the ST data $\mathrm{O}_{2}^{+}$cannot be distinguished uniquely from $\mathrm{S}^{+}$) and $\mathrm{CO}_{2}^{+}$, respectively, in the ST data. One can also see that the accumulated counts for the water group ions can exceed $10^{4}$ counts. For the water group ions we see the presence of several ghost peaks in the ST data; they occur at channels $\sim 200,475$, and about 1100 . The peaks at 200-250 channel numbers are probably $\mathrm{H}^{-}$ions coming out of the carbon foil of the IMS due to $\mathrm{OH}^{+}, \mathrm{H}_{2} \mathrm{O}^{+}, \mathrm{H}_{3} \mathrm{O}^{+}$, and $\mathrm{NH}_{3}^{+}$incident ions which have hydrogen in them. The peak at 400 to 500 channel numbers are probably due to $\mathrm{O}^{-}$ions coming out of the carbon foil of the IMS due to incident water group ions. The peaks at channel numbers near 1100 are the LEF ghost peaks due to incident water group ions and ammonia ions. At Dione $\mathrm{H}^{+}$peaks at around $80 \mathrm{eV}$ and occurs at ST channel 140 in TOF with its detectable LEF ghost peak at channel 260 (i.e., LEF ghost peak caused by $\mathrm{H}^{+}$ion striking LEF stop detector and secondary electron from LEF stop detector striking ST stop detector). Between $100 \mathrm{eV}$ and $200 \mathrm{eV}$ the ambient $\mathrm{H}_{2}^{+}$and pickup $\mathrm{H}_{2}^{+}$combine to form a single peak centered around ST channel 195; these ions would also be detectable using the LEF data. The water 
A

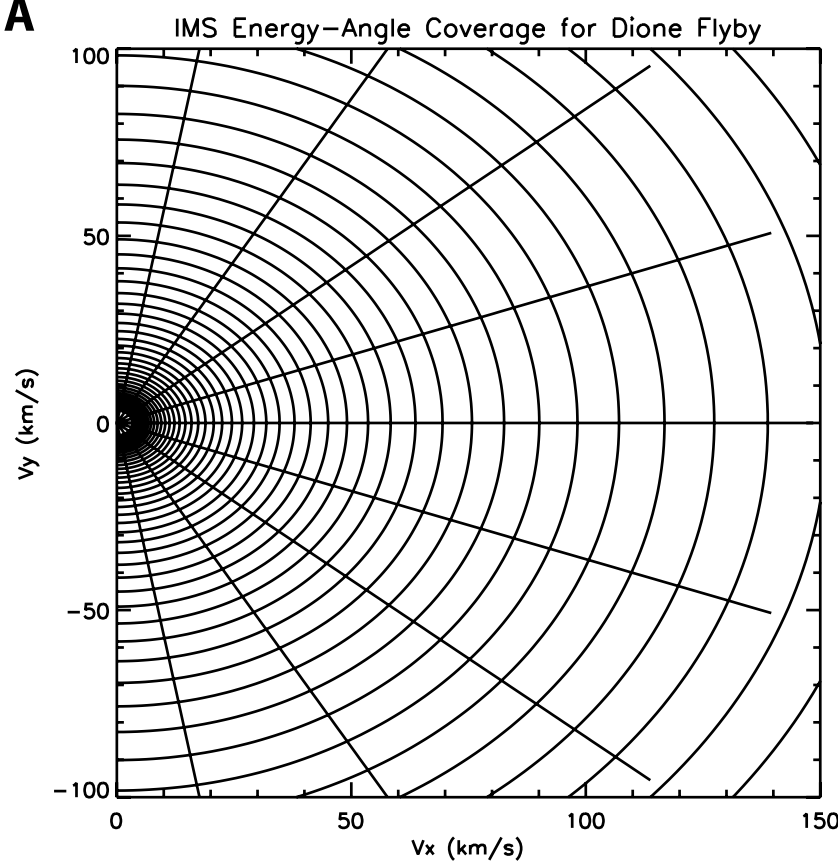

B

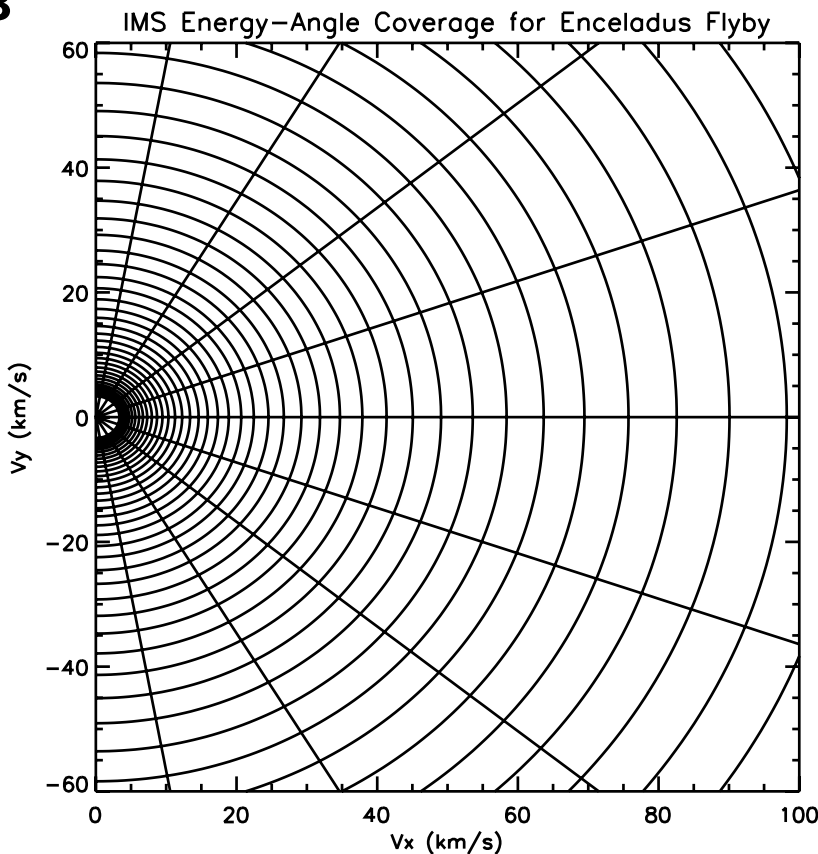

Figure 13. Example plot of IMS energy-angle coverage for pickup ions at (a) Dione and (b) Enceladus. The space between the lines defines the coverage of the instrument in velocity space for the specified ion, $\mathrm{E} / \mathrm{Q}$, and angle $\phi_{n}$ of the IMS.

group ions at Dione are centered around channel 250 and $900 \mathrm{eV}$ for the primary peak. $\mathrm{O}_{2}^{+}$peak occurs at around $1 \mathrm{keV}$ and ST channel 850 for Dione, while the $\mathrm{CO}_{2}^{+}$peak is around $2 \mathrm{keV}$ and ST channel 980 for Dione. In the case of Enceladus we see a similar result except that now the signal is confined below $1 \mathrm{keV}$, while for Dione counts extended up to $3 \mathrm{keV}$. This can be explained by the higher ambient corotation speeds and temperatures at Dione and correspondingly larger pickup energies at Dione.
[34] In the LEF data for Dione we see a clear peak for $\mathrm{CO}_{2}^{+}$at channel number 700 and $\mathrm{E} / \mathrm{Q} \sim 1-2 \mathrm{keV}$. At a slightly lower energy and LEF channel number of 950 1000 we see evidence of $\mathrm{O}_{2}^{+}$and $\mathrm{NH}_{3}^{+}$. At around $500 \mathrm{eV}$ we see clear evidence of $\mathrm{O}^{+}$and $\mathrm{H}_{2} \mathrm{O}^{+}$ions between LEF channels 1000 and 1100 . This data set will allow us to measure $\mathrm{NH}_{3}^{+}$. In the case of Enceladus the ion peaks are less pronounced, but we should be able to measure $\mathrm{NH}_{3}^{+}, \mathrm{H}_{3} \mathrm{O}^{+}$, $\mathrm{CO}_{2}^{+}$, and $\mathrm{O}_{2}^{+}$.

[35] For a more quantitative determination we consider several cuts of the ST contour maps at specific E/Q. For Dione we consider $\mathrm{E} / \mathrm{Q}=64 \mathrm{eV}, 724 \mathrm{eV}$, and $1450 \mathrm{eV}$ and are shown in Figure 16a. For Enceladus we consider E/Q = $64 \mathrm{eV}, 362 \mathrm{eV}$, and $1024 \mathrm{eV}$ and are displayed in Figure 17a. These cuts show the dominant presence of $\mathrm{H}^{+}$and $\mathrm{H}_{2}^{+}$ below $100 \mathrm{eV}$, while at $724 \mathrm{eV}$ for Dione and $362 \mathrm{eV}$ for Enceladus the spectra are dominated by water group ions and finally at $1450 \mathrm{eV}$ for Dione the dominant species are $\mathrm{O}_{2}^{+}$
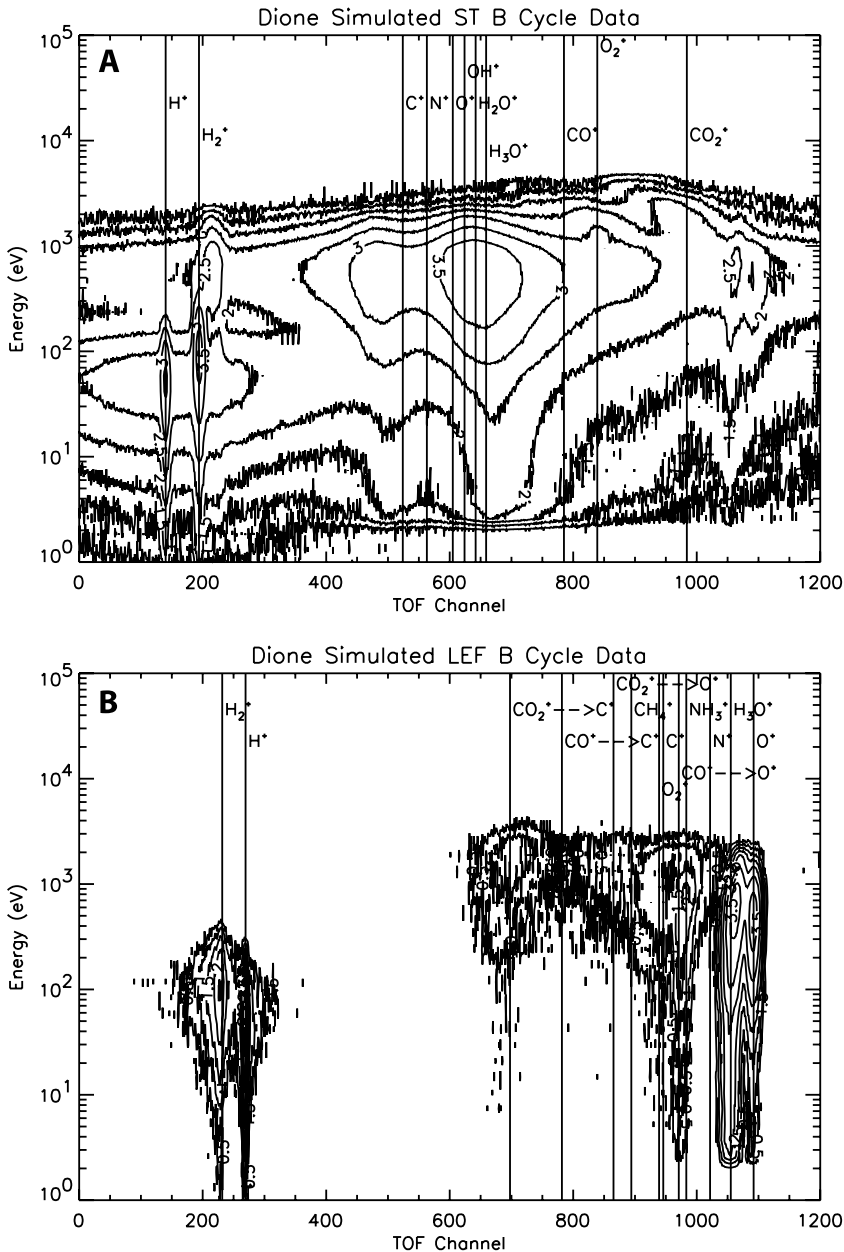

Figure 14. (a) Energy versus time-of-flight (TOF) contour map of simulated ions counts for B cycle ST data for Dione wake pass encounter. Vertical lines give approximate peak positions for various incident ions. The contour levels are $\log _{10}$ (ion counts). (b) Energy versus time-of-flight (TOF) contour map of simulated ions counts for B cycle LEF data for Dione encounter. Vertical lines give approximate peak positions for various incident ions. The contour levels are $\log _{10}$ (ion counts). 

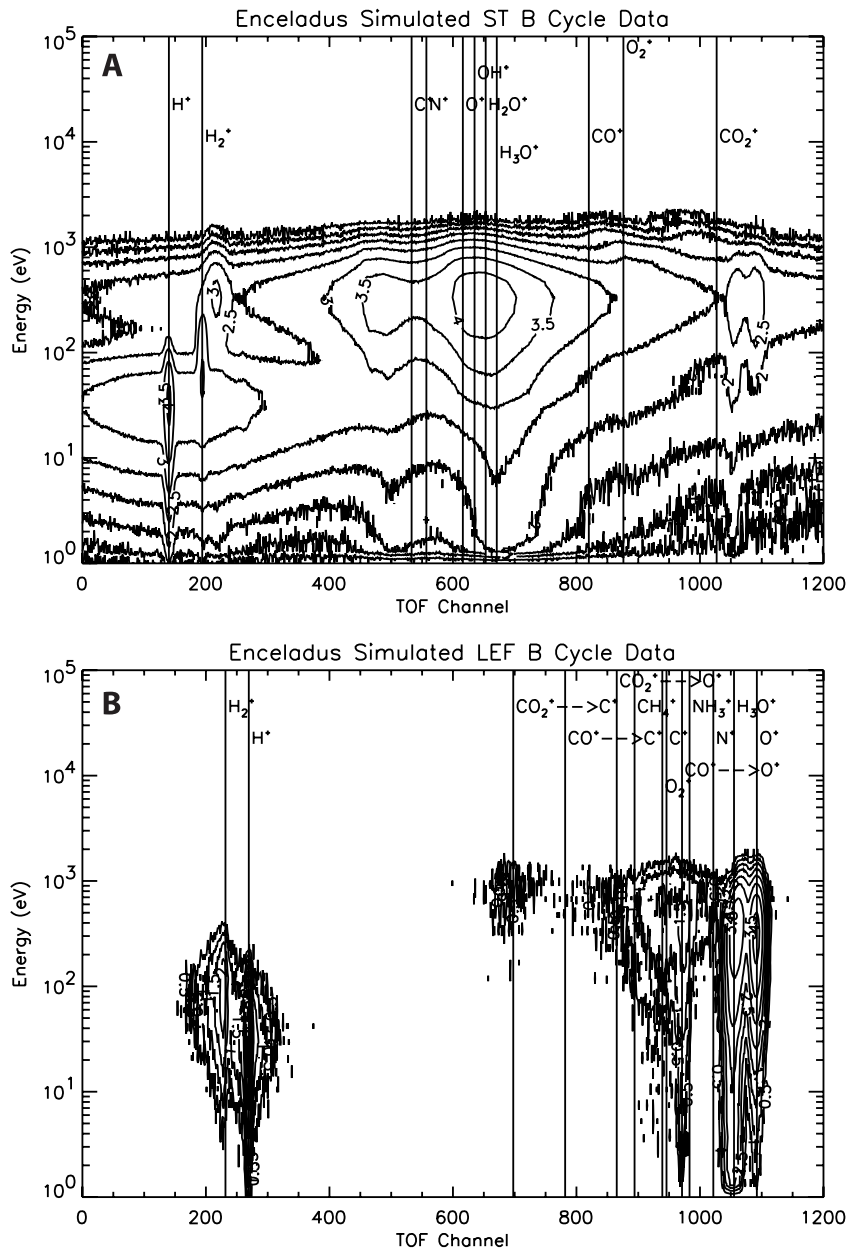

Figure 15. (a) Energy versus time-of-flight (TOF) contour map of simulated ions counts for B cycle ST data for Enceladus encounter. Vertical lines give approximate peak positions for various incident ions. The contour levels are $\log _{10}$ (ion counts). (b) Energy versus time-of-flight (TOF) contour map of simulated ions counts for B cycle LEF data for Enceladus encounter. Vertical lines give approximate peak positions for various incident ions. The contour levels are $\log _{10}$ (ion counts).

and $\mathrm{CO}_{2}^{+}$. For Enceladus, $\mathrm{O}_{2}^{+}$is important at $1024 \mathrm{eV}$ and $\mathrm{CO}_{2}^{+}$which is important at higher energies is barely seen at $1024 \mathrm{eV}$. At higher energies the peak at 200 to $250 \mathrm{ST}$ channels is the $\mathrm{H}^{-}$ghost peak due to water group ions; at ST channels 400 to 500 we are seeing $\mathrm{O}^{-}$ghost peaks from the water group ions. Between ST channels 1100 to 1200 we see the LEF ghost peaks due to the water group ions. For each individual cut of the spectrogram we can apply the SAM (Spectrum Analyzer Module) algorithm [see Sittler, 1993] and deconvolve the mass peaks to obtain a more accurate estimate of ion counts for each species present in the data. The application of this algorithm is no more accurate than the accuracy of the calibration data. In order to acquire the ion counts for various ion species at specific E/Q from this data set we need to use the SAM algorithm. For this analysis we used a total of 10 ions which required us to invert a $10 \times 10$ coupling coefficient matrix to get a $10 \times 10$ inverse coupling coefficient matrix (Note, each individual cut contains the accumulated counts for 14 ions. For our deconvolution algorithm we only used 10 of those ions and correspondingly only included the accumulated counts for those 10 ions.). We then bin the data (i.e., sum the ion counts for a range of TOF channels centered on ion peak in TOF) for 10 ion species and multiply the binned data by the inverse coupling coefficient matrix to get the ion counts for each individual ion species. The details of applying this algorithm are described by Sittler [1993].

[36] In Tables 5a and 5c we show for Dione and Enceladus, respectively, the deconvolved ion counts, $\left\langle\mathrm{C}_{\text {OUT }}\right\rangle$, with error bars, $\sigma_{\text {COUT }}$, for the various ion species derived from a subset of the ST simulated spectra similar to that shown in Figures $16 \mathrm{a}$ and $17 \mathrm{a}$. The $\left\langle\mathrm{C}_{\mathrm{OUT}}\right\rangle$ is an average computed from 40 solutions for which we added Poisson
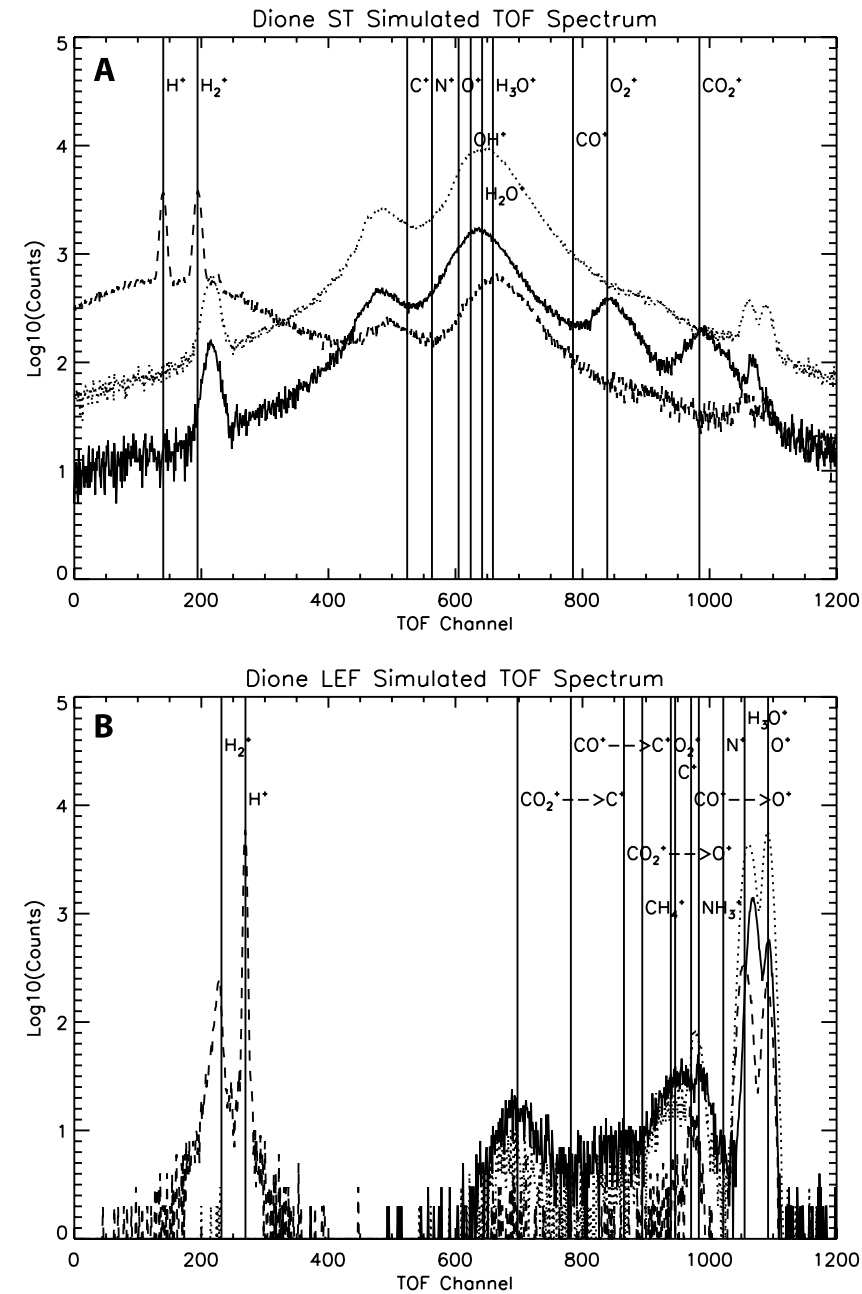

Figure 16. (a) Simulated ion counts versus TOF for B cycle ST data for Dione encounter. Each curve is a cut through the energy-TOF spectrogram at $\mathrm{E} / \mathrm{Q}=64 \mathrm{eV}$ (dashed line), $724 \mathrm{eV}$ (dotted line) and $1450 \mathrm{eV}$ (solid line). Vertical lines give approximate peak positions for various incident ions. (b) Simulated ion counts versus TOF for B cycle LEF data for Dione encounter. Each curve is a cut through the energy-TOF spectrogram at $\mathrm{E} / \mathrm{Q}=64 \mathrm{eV}$ (dashed line), $724 \mathrm{eV}$ (dotted line) and $1450 \mathrm{eV}$ (solid line). Vertical lines give approximate peak positions for various incident ions. 


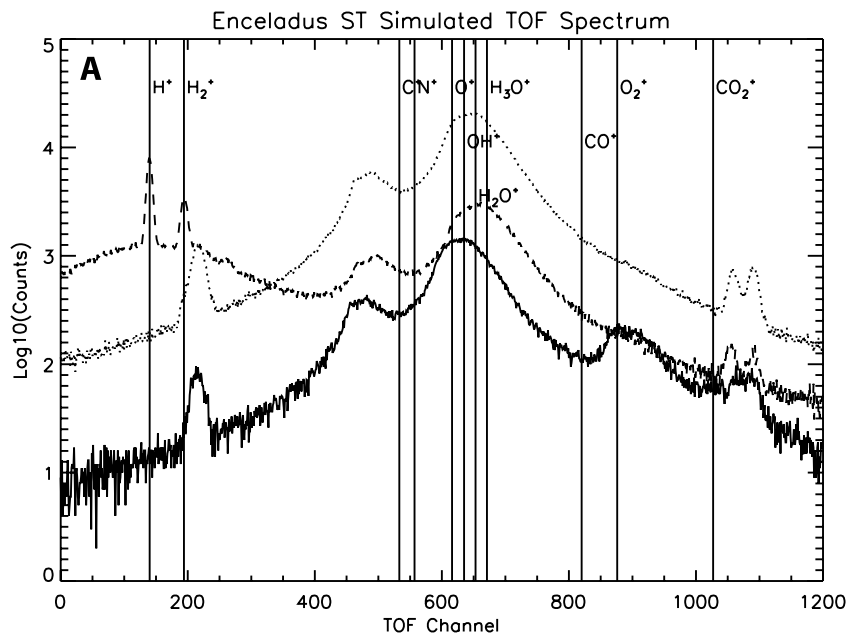

Table 5a. Dione SAM ST Derived Ion Counts

\begin{tabular}{|c|c|c|c|c|c|c|}
\hline $\begin{array}{l}\text { Ion } \\
\text { Type }\end{array}$ & $\begin{array}{c}\mathrm{E} / \mathrm{Q}, \\
\mathrm{eV}\end{array}$ & Resolution & $\begin{array}{c}\mathrm{C}_{\mathrm{IN}} \\
\text { (ambient) }\end{array}$ & $\begin{array}{c}\mathrm{C}_{\mathrm{IN}} \\
\text { (ambient }+ \\
\text { ring) }\end{array}$ & $\left\langle\mathrm{C}_{\text {OUT }}\right\rangle$ & $\sigma_{\text {COUT }}$ \\
\hline $\mathrm{H}^{+}$ & 1450.0 & ST & 0.0 & 0.0 & -1.18 & 9.13 \\
\hline “ & 724.0 & “ & 0.0 & 0.0 & -12.6 & 31.2 \\
\hline “ & 64.0 & “ & $2.45 \mathrm{E}+5$ & $2.61 \mathrm{E}+5$ & $2.61 \mathrm{E}+5$ & 648.0 \\
\hline $\mathrm{H}_{2}^{+}$ & 1450.0 & ST & 0.0 & 0.0 & -0.795 & 16.1 \\
\hline “ & 724.0 & "“ & 66.2 & 66.2 & 62.0 & 69.3 \\
\hline “ & 64.0 & " & $3.91 \mathrm{E}+4$ & $4.87 \mathrm{E}+4$ & $4.85 \mathrm{E}+4$ & 461.0 \\
\hline $\mathrm{O}^{+}$ & 1450.0 & ST & $2.12 \mathrm{E}+4$ & $2.31 \mathrm{E}+4$ & $2.41 \mathrm{E}+4$ & 5196.0 \\
\hline “ & 724.0 & “ & $7.88 \mathrm{E}+5$ & $8.11 \mathrm{E}+5$ & $8.11 \mathrm{E}+5$ & $1.36 \mathrm{E}+4$ \\
\hline “ & 64.0 & “ & $4.48 \mathrm{E}+4$ & $4.92 \mathrm{E}+4$ & $4.81 \mathrm{E}+4$ & 3588.0 \\
\hline $\mathrm{OH}^{+}+\mathrm{NH}_{3}^{+}$ & 1450.0 & ST & $1.25 \mathrm{E}+4$ & $2.44 \mathrm{E}+4$ & $2.18 \mathrm{E}+4$ & 11306.0 \\
\hline “ & 724.0 & “ & $3.06 \mathrm{E}+5$ & $3.66 \mathrm{E}+5$ & $3.64 \mathrm{E}+5$ & $2.47 \mathrm{E}+4$ \\
\hline “ & 64.0 & “ & $1.48 \mathrm{E}+4$ & $2.86 \mathrm{E}+4$ & $3.04 \mathrm{E}+4$ & 6556.0 \\
\hline $\mathrm{H}_{2} \mathrm{O}^{+}$ & 1450.0 & ST & $1.47 \mathrm{E}+4$ & $8.90 \mathrm{E}+4$ & $9.2 \mathrm{E}+4$ & 12211.0 \\
\hline & 724.0 & “ & $2.51 \mathrm{E}+5$ & $6.26 \mathrm{E}+5$ & $6.28 \mathrm{E}+5$ & $2.24 \mathrm{E}+4$ \\
\hline “ & 64.0 & “ & $1.05 \mathrm{E}+4$ & $5.86 \mathrm{E}+4$ & $5.69 \mathrm{E}+4$ & 6063.0 \\
\hline $\mathrm{H}_{3} \mathrm{O}^{+}$ & 1450.0 & ST & 0.0 & $3.68 \mathrm{E}+3$ & $1.95 \mathrm{E}+3$ & $6.46 \mathrm{E}+3$ \\
\hline “ & 724.0 & “" & 0.0 & $1.85 \mathrm{E}+4$ & $1.71 \mathrm{E}+4$ & $1.04 \mathrm{E}+4$ \\
\hline “" & 64.0 & “ & 0.0 & 2357.0 & 3048.0 & 2895.0 \\
\hline $\mathrm{O}_{2}^{+}$ & 1450.0 & ST & 6373.0 & $3.05 \mathrm{E}+4$ & $3.05 \mathrm{E}+4$ & 264.0 \\
\hline “" & 724.0 & “ & 7445.0 & $2.17 \mathrm{E}+4$ & $2.17 \mathrm{E}+4$ & 287.0 \\
\hline “ & 64.0 & “" & 120.0 & 2250.0 & 2236.0 & 109.0 \\
\hline $\mathrm{CO}_{2}^{+}$ & 1450.0 & ST & 0.0 & $2.35 \mathrm{E}+4$ & $2.35 \mathrm{E}+4$ & 249.0 \\
\hline “ & 724.0 & “ & 0.0 & 7811.0 & 7770.0 & 233.0 \\
\hline “" & 64.0 & "“ & 0.0 & 1991.0 & 1969.0 & 83.1 \\
\hline $\mathrm{CO}^{+}$ & 1450.0 & ST & 0.0 & 681.0 & 673.0 & 181.0 \\
\hline “ & & “ & 0.0 & 482.0 & 431.0 & 254.0 \\
\hline “ & 64.0 & “" & 0.0 & 73.0 & 46.9 & 88.1 \\
\hline
\end{tabular}

$\mathrm{CO}_{2}^{+}$, and $\mathrm{CO}^{+}$we maybe able to separate them from the ambient ions when using the $\mathrm{B}$ cycle data when combined with the A cycle data. The primary reason for the ambiguity in the B cycle data is because we add the eight angular

Table 5b. Dione SAM LEF Derived Ion Counts

Figure 17. (a) Simulated ion counts versus TOF for B cycle ST data for Enceladus encounter. Each curve is a cut through the energy-TOF spectrogram at $\mathrm{E} / \mathrm{Q}=64 \mathrm{eV}$ (dashed line), $362 \mathrm{eV}$ (dotted line) and $1024 \mathrm{eV}$ (solid line). Vertical lines give approximate peak positions for various incident ions. (b) Simulated ion counts versus TOF for B cycle LEF data for Enceladus encounter. Each curve is a cut through the energy-TOF spectrogram at $\mathrm{E} / \mathrm{Q}=64 \mathrm{eV}$ (dashed line), $362 \mathrm{eV}$ (dotted line) and $1024 \mathrm{eV}$ (solid line). Vertical lines give approximate peak positions for various incident ions.

statistical noise to the simulated TOF spectra. The same can be said for our estimate of the standard deviation $\sigma_{\text {COUT }}$ The tables also show the input counts $\mathrm{C}_{\mathrm{IN}}$ for ambient ions only and ambient ions plus pickup ions. We show the ambient only counts to provide a baseline to determine our ability to separate the ambient ions from the pickup ions. In most cases $\left\langle\mathrm{C}_{\text {OUT }}\right\rangle \approx \mathrm{C}_{\mathrm{IN}}$ (ambient + ring). The results show that one can separate the water group ions very well and shows the power of our deconvolution algorithm, which we refer to as the SAM algorithm. For the water group ions the errors range from $1 \%$ to $6 \%$. By comparing the ambient only and ambient plus pickup ion counts one can see that with the $\mathrm{B}$ cycle data it will be difficult to separate the pickup ions from the ambient ions for $\mathrm{H}^{+}, \mathrm{H}_{2}^{+}$, and $\mathrm{O}^{+}$. For the remaining ions $\mathrm{OH}^{+}, \mathrm{H}_{2} \mathrm{O}^{+}, \mathrm{H}_{3} \mathrm{O}^{+}, \mathrm{O}_{2}^{+}$,

\begin{tabular}{|c|c|c|c|c|c|c|}
\hline $\begin{array}{c}\text { Ion } \\
\text { Type }\end{array}$ & $\begin{array}{c}\mathrm{E} / \mathrm{Q}, \\
\mathrm{eV}\end{array}$ & Resolution & $\begin{array}{c}\mathrm{C}_{\mathrm{IN}} \\
\text { (ambient) }\end{array}$ & $\begin{array}{c}\mathrm{C}_{\mathrm{IN}} \\
\text { (ambient }+ \\
\text { ring) }\end{array}$ & $\left\langle\mathrm{C}_{\text {OUT }}\right\rangle$ & $\sigma_{\mathrm{COUT}}$ \\
\hline $\mathrm{H}^{+}$ & 1450.0 & LEF & 0.0 & 0.0 & 0.1 & 0.74 \\
\hline “ & 724.0 & “ & 0.0 & 0.0 & 0.03 & 0.48 \\
\hline ، & 64.0 & “ & $2.45 \mathrm{E}+4$ & $2.61 \mathrm{E}+4$ & $2.6 \mathrm{E}+4$ & 172.0 \\
\hline $\mathrm{H}_{2}^{+}$ & 1450.0 & LEF & 0.0 & 0.0 & 0.23 & 1.2 \\
\hline ، & 724.0 & “" & 6.62 & 6.62 & 6.14 & 2.78 \\
\hline “ & 64.0 & “ & 3907.0 & 4872.0 & 4887.0 & 104.0 \\
\hline $\mathrm{NH}_{3}^{+}$ & 1450.0 & LEF & 0.0 & 271.0 & 266.0 & 20.7 \\
\hline “ & 724.0 & “ & 0.0 & 1360.0 & 1355.0 & 49.7 \\
\hline ، & 64.0 & “ & 0.0 & 313.0 & 306.0 & 19.3 \\
\hline $\mathrm{O}^{+}$ & 1450.0 & LEF & 2118.0 & 2309.0 & 2320.0 & 67.3 \\
\hline “ & 724.0 & “ & $7.88 \mathrm{E}+4$ & $8.11 \mathrm{E}+4$ & $8.11 \mathrm{E}+4$ & 313.0 \\
\hline “ & 64.0 & “ & 4475.0 & 4923.0 & 4914.0 & 76.7 \\
\hline $\mathrm{OH}^{+}$ & 1450.0 & LEF & 1246.0 & 2168.0 & 2148.0 & 171.0 \\
\hline “ & 724.0 & “" & $3.06 \mathrm{E}+4$ & $3.52 \mathrm{E}+4$ & $3.51 \mathrm{E}+4$ & 422.0 \\
\hline ، & 64.0 & “ & 1476.0 & 2542.0 & 2578.0 & 117.0 \\
\hline $\mathrm{H}_{2} \mathrm{O}^{+}$ & 1450.0 & LEF & 1470.0 & 8899.0 & 8878.0 & 205.0 \\
\hline “" & 724.0 & ، & $2.51 \mathrm{E}+4$ & $6.26 \mathrm{E}+4$ & $6.27 \mathrm{E}+4$ & 526.0 \\
\hline “ & 64.0 & “ & 1054.0 & 5859.0 & 5819.0 & 160.0 \\
\hline $\mathrm{H}_{3} \mathrm{O}^{+}$ & 1450.0 & LEF & 0.0 & 368.0 & 379.0 & 100.0 \\
\hline “ & 724.0 & “ & 0.0 & 1851.0 & 1873.0 & 292.0 \\
\hline “ & 64.0 & “" & 0.0 & 236.0 & 250.0 & 67.3 \\
\hline $\mathrm{O}_{2}^{+}$ & 1450.0 & LEF & 637.0 & 3049.0 & 3039.0 & 69.4 \\
\hline " & 724.0 & “" & 745.0 & 2168.0 & 2163.0 & 52.6 \\
\hline “" & 64.0 & “ & 12.0 & 225.0 & 223.0 & 22.2 \\
\hline $\mathrm{CO}_{2}^{+}$ & 1450.0 & LEF & 0.0 & 2350.0 & 2337.0 & 73.9 \\
\hline " & 724.0 & “ & 0.0 & 781.0 & 786.0 & 48.1 \\
\hline “ & 64.0 & “ & 0.0 & 199.0 & 199.0 & 27.0 \\
\hline $\mathrm{CO}^{+}$ & 1450.0 & LEF & 0.0 & 68.0 & 70.4 & 30.4 \\
\hline “ & 724.0 & “ & 0.0 & 48.2 & 46.4 & 19.5 \\
\hline “ & 64.0 & “ & 0.0 & 7.3 & 7.84 & 10.0 \\
\hline
\end{tabular}


Table 5c. Enceladus SAM ST Derived Ion Counts

\begin{tabular}{|c|c|c|c|c|c|c|}
\hline $\begin{array}{c}\text { Ion } \\
\text { Type }\end{array}$ & $\begin{array}{c}\mathrm{E} / \mathrm{Q}, \\
\mathrm{eV}\end{array}$ & Resolution & $\begin{array}{c}\mathrm{C}_{\mathrm{IN}} \\
\text { (ambient) }\end{array}$ & $\begin{array}{c}\mathrm{C}_{\mathrm{IN}} \\
\text { (ambient }+ \\
\text { ring) }\end{array}$ & $\left\langle\mathrm{C}_{\text {OUT }}\right\rangle$ & $\sigma_{\text {COUT }}$ \\
\hline $\mathrm{H}^{+}$ & 1024 & ST & 0.0 & 0.0 & 0.175 & 6.24 \\
\hline “ & 362 & " & 0.0 & 0.0 & -9.92 & 44.9 \\
\hline “ & 64 & “ & $4.29 \mathrm{E}+5$ & $4.29 \mathrm{E}+5$ & $4.28 \mathrm{E}+5$ & 853.0 \\
\hline $\mathrm{H}_{2}^{+}$ & 1024 & ST & 0.0 & 0.0 & 5.52 & 18.0 \\
\hline “" & 362 & “ & 456.0 & 456.0 & 410.0 & 108.0 \\
\hline “ & 64 & “" & $3.10 \mathrm{E}+4$ & $3.15 \mathrm{E}+4$ & $3.16 \mathrm{E}+4$ & 425.0 \\
\hline $\mathrm{O}^{+}$ & 1024 & ST & $4.49 \mathrm{E}+4$ & $4.49 \mathrm{E}+4$ & $4.59 \mathrm{E}+4$ & 4196.0 \\
\hline “ & 362 & “ & $2.01 \mathrm{E}+6$ & $2.03 \mathrm{E}+6$ & $2.02 \mathrm{E}+6$ & $3.58 \mathrm{E}+4$ \\
\hline “ & 64 & “ & $3.64 \mathrm{E}+5$ & $3.67 \mathrm{E}+5$ & $3.69 \mathrm{E}+5$ & 8815.0 \\
\hline $\mathrm{OH}^{+}+\mathrm{NH}_{3}^{+}$ & 1024 & ST & $1.4 \mathrm{E}+4$ & $1.4 \mathrm{E}+4$ & $1.18 \mathrm{E}+4$ & 9049.0 \\
\hline “ & 362 & " & $4.21 \mathrm{E}+5$ & $4.51 \mathrm{E}+5$ & $4.63 \mathrm{E}+5$ & $6.76 \mathrm{E}+4$ \\
\hline “" & 64 & “ & $6.47 \mathrm{E}+4$ & $7.39 \mathrm{E}+4$ & $7.05 \mathrm{E}+4$ & $1.7 \mathrm{E}+4$ \\
\hline $\mathrm{H}_{2} \mathrm{O}^{+}$ & 1024 & $\mathrm{ST}$ & $2.53 \mathrm{E}+4$ & $2.53 \mathrm{E}+4$ & $2.73 \mathrm{E}+4$ & 9462.0 \\
\hline “" & 362 & “ & $6.24 \mathrm{E}+5$ & $1.23 \mathrm{E}+6$ & $1.22 \mathrm{E}+6$ & $5.84 \mathrm{E}+4$ \\
\hline ، & 64 & “ & $9.56 \mathrm{E}+4$ & $2.8 \mathrm{E}+5$ & $2.83 \mathrm{E}+5$ & $1.59 \mathrm{E}+4$ \\
\hline $\mathrm{H}_{3} \mathrm{O}^{+}$ & 1024 & ST & 0.0 & 0.0 & -817.0 & 4819.0 \\
\hline “" & 362 & “ & 0.0 & $2.03 \mathrm{E}+4$ & $2.07 \mathrm{E}+4$ & $2.6 \mathrm{E}+4$ \\
\hline “ & 64 & “ & 0.0 & 6069.0 & 5429.0 & 7592.0 \\
\hline $\mathrm{O}_{2}^{+}$ & 1024 & ST & 3528.0 & $1.57 \mathrm{E}+4$ & $1.57 \mathrm{E}+4$ & 241.0 \\
\hline “ & 362 & " & 5381.0 & $2.3 \mathrm{E}+4$ & $2.31 \mathrm{E}+4$ & 315.0 \\
\hline “ & 64 & “ & 267.0 & 5572.0 & 5506.0 & 138.0 \\
\hline $\mathrm{CO}_{2}^{+}$ & 1024 & ST & 0.0 & 5565.0 & 5551.0 & 110.0 \\
\hline “ & 362 & “ & 0.0 & 3290.0 & 3329.0 & 281.0 \\
\hline “ & 64 & “ & 0.0 & 538.0 & 547.0 & 194.0 \\
\hline $\mathrm{CO}^{+}$ & 1024 & $\mathrm{ST}$ & 0.0 & 18.5 & 8.4 & 71.1 \\
\hline “" & 362 & “ & 0.0 & 74.9 & 68.0 & 324.0 \\
\hline “ & 64 & “" & 0.0 & 32.3 & 18.7 & 152.0 \\
\hline
\end{tabular}

sectors of the IMS together in the B cycle data. In the A cycle data we retain the energy-angle dependence of the ion counts. The advantage of the $\mathrm{B}$ cycle data is its greater sensitivity. Well-determined ion counts for $\mathrm{H}^{+}, \mathrm{H}_{2}^{+}, \mathrm{O}_{2}^{+}$, and $\mathrm{CO}_{2}^{+}$are estimated (i.e., errors $\sim 1 \%$ ) because these species are well separated from each other and the water group ions. $\mathrm{NH}_{3}^{+}$overlaps with $\mathrm{OH}^{+}$and we are not able to distinguish between the two ions (i.e., one exception is the $\mathrm{OH}^{+}$will produce an $\mathrm{O}^{-}$ghost peak and $\mathrm{NH}_{3}^{+}$will not). The detection of $\mathrm{H}_{3} \mathrm{O}^{+}$has a large uncertainty $\sim 60 \%$ due to its colocation with the ambient ions $\mathrm{O}^{+}, \mathrm{H}_{2} \mathrm{O}^{+}$, and $\mathrm{OH}^{+}$.

[37] The LEF simulated B cycle data, Figures 14b and $15 \mathrm{~b}$, shows singular peaks for atomic $\mathrm{H}^{+}, \mathrm{H}_{2}{ }^{+}, \mathrm{H}_{2} \mathrm{O}^{+}$, and $\mathrm{O}^{+}$. The simulations also show shifted peak positions due to the presence of molecules; for example, the figure shows shifted peak for $\mathrm{C}^{+}$and $\mathrm{O}^{+}$for which the parent molecule is $\mathrm{CO}_{2}^{+}$; $\mathrm{CO}^{+}$is present but is not resolved because of its low abundance relative to the other species. This shift results from the molecule breaking up in the carbon foil such that the exit speed from the carbon foil of the fragments is equal to the entrance speed of the parent molecule to the carbon foil. This causes a $15 \mathrm{keV} \mathrm{CO}^{+}$incident on carbon foil to have $\mathrm{C}^{+}$ion of energy $6.42 \mathrm{keV}$ exiting carbon foil so that the fragment has sufficiently low enough energy that the detuning effect of the LEF section is important [see Nordholt et al., 1998]. The energy dependence of LEF molecular peaks is that the peak position shifts to lower TOF with decreasing ion exit energy from the carbon foil. In order to calibrate our simulations we have taken as a standard, calibration data for $\mathrm{N}^{+}$and combined with the calibration data for $\mathrm{N}_{2}^{+}$by Nordholt et al. [1998] to extend the coverage below $15 \mathrm{keV}$. For E/Q $>15 \mathrm{keV}$ we fit the data with a power law in energy (i.e., TOF $=a^{*} \mathrm{E}^{\widehat{c}} \mathrm{c}+\mathrm{b}$ where $\mathrm{a}, \mathrm{b}$, and $\mathrm{c}$ are determined from numerical fits to calibration data), while for $\mathrm{E} / \mathrm{Q} \leq 15 \mathrm{kV}$ we have fit the data with polynomial of order 3 (i.e., $\mathrm{TOF}=\mathrm{ap}+\mathrm{bp} * \mathrm{E}+\mathrm{cp}^{*} \mathrm{E}^{\widehat{2}} 2+$ $\mathrm{dp}^{*} \mathrm{E}^{\wedge} 3$ for which $\mathrm{ap}, \mathrm{bp}, \mathrm{cp}$, and $\mathrm{dp}$ are determined from numerical fits to calibration data). For the other ions we then multiply the above expression by $\operatorname{sqr}(\mathrm{M} / 14)$ where $M$ is the mass of the fragment exiting the carbon foil. In the case of $\mathrm{CO}^{+} \rightarrow \mathrm{C}^{+}$, the mass $\mathrm{M}=12$ and energy $E=E_{C^{+}}(12 / 28)$ which is then substituted into our polynomial expression for TOF. In reality more detailed calibration data will be required under real conditions. These results are accurate enough to display the effects expected based on instrument response.

[38] Using the data from Nordholt et al.'s [1998] Figure 6 we have also estimated the probability for a $\mathrm{C}^{+}$fragment $(\mathrm{P}=0.79)$ relative to that for an $\mathrm{O}^{+}$fragment $(\mathrm{P}=0.21)$ for which the parent molecule is $\mathrm{CO}^{+}$. The fact that $\mathrm{P}\left(\mathrm{O}^{+}\right) \ll$ $\mathrm{P}\left(\mathrm{C}^{+}\right)$indicates the higher affinity that oxygen has for electrons relative to that for carbon. A similar calculation can be done for $\mathrm{CO}_{2}^{+}$for which $\mathrm{P}=0.65$ for $\mathrm{C}^{+}$and $\mathrm{P}=0.35$ for $\mathrm{O}^{+}$. Inspection of Figure $14 \mathrm{~b}$ shows no evidence of $\mathrm{CO}^{+}$ at TOF $\sim 780$ which means that the peak at about TOF $\sim$ 980 is due to $\mathrm{NH}_{3}^{+}$. One can see from these same figures the shift of $\mathrm{H}_{2}^{+}$from $\mathrm{H}^{+}$to lower TOF so that it is well separated from the $\mathrm{H}^{+}$peak. We also see a well-separated peak for $\mathrm{NH}_{3}^{+}$from $\mathrm{N}^{+}$and $\mathrm{H}_{2} \mathrm{O}^{+}$from $\mathrm{O}^{+}$. We also see a well-resolved peak for $\mathrm{O}_{2}^{+} \rightarrow \mathrm{O}^{+}$; because of this we can uniquely separate $\mathrm{O}_{2}^{+}$from $\mathrm{S}^{+}$. While for the ST simulations this separation of peaks is not always possible. The simulations also underscore the importance of having

Table 5d. Enceladus SAM LEF Derived Ion Counts

\begin{tabular}{|c|c|c|c|c|c|c|}
\hline $\begin{array}{c}\text { Ion } \\
\text { Type }\end{array}$ & $\begin{array}{c}\mathrm{E} / \mathrm{Q}, \\
\mathrm{eV}\end{array}$ & Resolution & $\begin{array}{c}\mathrm{C}_{\mathrm{IN}} \\
\text { (ambient) }\end{array}$ & $\begin{array}{c}\mathrm{C}_{\mathrm{IN}} \\
\text { (ambient }+ \\
\text { ring) }\end{array}$ & $\left\langle\mathrm{C}_{\text {OUT }}\right\rangle$ & $\sigma_{\mathrm{COUT}}$ \\
\hline $\mathrm{H}^{+}$ & 1024 & LEF & 0.0 & 0.0 & 0.0 & 0.3 \\
\hline “ & 362 & “ & 0.0 & 0.0 & 0.24 & 0.72 \\
\hline ، & 64 & “ & $2.12 \mathrm{E}+6$ & $4.29 \mathrm{E}+4$ & $4.29 \mathrm{E}+4$ & 228.0 \\
\hline $\mathrm{H}_{2}^{+}$ & 1024 & LEF & 0.0 & 0.0 & 0.0 & 0.44 \\
\hline " & 362 & “" & 45.6 & 45.6 & 47.2 & 9.3 \\
\hline “ & 64 & “ & 3096.0 & 3150.0 & 3155.0 & 70.8 \\
\hline $\mathrm{NH}_{3}{ }^{+}$ & 1024 & LEF & 0.0 & 0.0 & 4.58 & 11.4 \\
\hline “ & 362 & “ & 0.0 & 879.0 & 879.0 & 36.8 \\
\hline ، & 64 & “ & 0.0 & 265.0 & 262.0 & 24.7 \\
\hline $\mathrm{O}^{+}$ & 1024 & LEF & 4486.0 & 4486.0 & 4470.0 & 96.1 \\
\hline “ & 362 & “ & $2.01 \mathrm{E}+5$ & $2.03 \mathrm{E}+5$ & $2.03 E+5$ & 530.0 \\
\hline “ & 64 & “ & $3.64 \mathrm{E}+4$ & $3.67 \mathrm{E}+4$ & $3.67 \mathrm{E}+4$ & 233.0 \\
\hline $\mathrm{OH}^{+}$ & 1024 & LEF & 1402.0 & 1402.0 & 1387.0 & 114.0 \\
\hline “ & 362 & “ & $4.21 \mathrm{E}+4$ & $4.42 \mathrm{E}+4$ & $4.41 \mathrm{E}+4$ & 627.0 \\
\hline ، & 64 & “ & 6471.0 & 7125.0 & 7108.0 & 242.0 \\
\hline $\mathrm{H}_{2} \mathrm{O}^{+}$ & 1024 & LEF & 3095.0 & 3095.0 & 3102.0 & 145.0 \\
\hline “ & 362 & “ & $6.5 \mathrm{E}+4$ & $1.25 \mathrm{E}+5$ & $1.25 \mathrm{E}+5$ & 763.0 \\
\hline ، & 64 & “ & 8679.0 & $2.67 \mathrm{E}+4$ & $2.67 \mathrm{E}+4$ & 325.0 \\
\hline $\mathrm{H}_{3} \mathrm{O}^{+}$ & 1024 & LEF & 0.0 & 0.0 & -6.21 & 68.2 \\
\hline “ & 362 & “ & 0.0 & 2031.0 & 1919.0 & 470.0 \\
\hline ، & 64 & ، & 0.0 & 607.0 & 594.0 & 181.0 \\
\hline $\mathrm{O}_{2}^{+}$ & 1024 & LEF & 353.0 & 1570.0 & 1566.0 & 43.1 \\
\hline " & 362 & “" & 538.0 & 2304.0 & 2311.0 & 61.7 \\
\hline “ & 64 & “ & 26.7 & 557.0 & 557.0 & 24.9 \\
\hline $\mathrm{CO}_{2}^{+}$ & 1024 & LEF & 0.0 & 556.0 & 555.0 & 36.5 \\
\hline " & 362 & " & 0.0 & 329.0 & 342.0 & 26.0 \\
\hline “ & 64 & “ & 0.0 & 53.8 & 55.2 & 11.4 \\
\hline $\mathrm{CO}^{+}$ & 1024 & LEF & 0.0 & 1.85 & 5.2 & 12.4 \\
\hline “" & 362 & " & 0.0 & 7.5 & 5.25 & 13.6 \\
\hline “ & 64 & “ & 0.0 & 3.23 & 3.96 & 6.67 \\
\hline
\end{tabular}


good calibration data. In some instances the ST data can better separate the ions of different M/Q, while the LEF data is best for separating atomic and molecular species of similar M/Q. It should also be emphasized that in many cases the LEF feature of the IMS will provide a unique separation of molecules from molecules and atomic ions of the same M/Q. For example, the Nordholt et al. [1998] results in Figure 6 of that paper show how the $\mathrm{CO}^{+} \rightarrow \mathrm{C}^{+}$ and $\mathrm{CO}^{+} \rightarrow \mathrm{O}^{+}$peaks are well separated from the $\mathrm{N}_{2}^{+} \rightarrow \mathrm{N}^{+}$ peak; for which $\mathrm{CO}^{+}$and $\mathrm{N}_{2}^{+}$have the same M/Q. Also, ${ }^{40} \mathrm{Ar}$ will be well separated from $\mathrm{CO}_{2}{ }^{+}$which has $\mathrm{M} / \mathrm{Q}=44$. It should be clear from these results that CAPS should be able to separate the molecules from the corresponding atomic species. Again, the main difference between Figure 14b and Figure $15 \mathrm{~b}$ is that for Enceladus the ion peaks are confined to lower energy relative to that for Dione. Because the ions are measured at lower energy for Enceladus the LEF TOF peaks will be shifted to lower TOF for Enceladus relative to that for Dione. In the case of the ST data the inverse is true. Here, we emphasize the fact that the water group ions are not well resolved in the ST data while for the LEF data they are resolved to a greater extent.

[39] In Figures $16 \mathrm{~b}$ and $17 \mathrm{~b}$ we show cuts through the LEF E/Q versus TOF spectrograms for Dione and Enceladus, respectively, with $\mathrm{E} / \mathrm{Q}=64 \mathrm{eV}, 724 \mathrm{eV}$, and $1450 \mathrm{eV}$ for Dione and $\mathrm{E} / \mathrm{Q}=64 \mathrm{eV}, 362 \mathrm{eV}$, and $1024 \mathrm{eV}$ for Enceladus. For Dione we see at E/Q $<100 \mathrm{eV}$ well-resolved peaks for $\mathrm{H}^{+}, \mathrm{H}_{2}^{+}, \mathrm{NH}_{3}^{+} \rightarrow \mathrm{N}^{+}, \mathrm{H}_{2} \mathrm{O}^{+} \rightarrow \mathrm{O}^{+}$, and $\mathrm{O}^{+}$. At $\mathrm{E} / \mathrm{Q}=724 \mathrm{eV}$ for Dione we see well-resolved peaks for $\mathrm{CO}_{2}^{+} \rightarrow \mathrm{C}^{+}, \mathrm{CO}_{2}^{+} \rightarrow \mathrm{O}^{+}, \mathrm{O}_{2}^{+} \rightarrow \mathrm{O}^{+}, \mathrm{NH}_{3}^{+} \rightarrow \mathrm{N}^{+}, \mathrm{H}_{2} \mathrm{O}^{+} \rightarrow$ $\mathrm{O}^{+}$, and $\mathrm{O}^{+}$. These spectra show the power of the LEF feature of the IMS in that the species $\mathrm{NH}_{3}^{+}, \mathrm{N}^{+}, \mathrm{H}_{2} \mathrm{O}^{+}$, and $\mathrm{O}^{+}$can be separated, while for ST data it would not be possible to do very accurately. At $\mathrm{E} / \mathrm{Q}=1450 \mathrm{eV}$ for Dione the spectrum is dominated by peaks for $\mathrm{CO}_{2}^{+} \rightarrow \mathrm{C}^{+}, \mathrm{CO}_{2}^{+} \rightarrow$ $\mathrm{O}^{+}, \mathrm{O}_{2}^{+} \rightarrow \mathrm{O}^{+}, \mathrm{NH}_{3}^{+} \rightarrow \mathrm{N}^{+}, \mathrm{H}_{2} \mathrm{O}^{+} \rightarrow \mathrm{O}^{+}$, and $\mathrm{O}^{+} \rightarrow \mathrm{O}^{+}$. The cuts in E/Q for Enceladus show peaks for $\mathrm{H}^{+}, \mathrm{H}_{2}{ }^{+}$at $64 \mathrm{eV}$ and peaks for $\mathrm{O}_{2}{ }^{+} \rightarrow \mathrm{O}^{+}, \mathrm{NH}_{3}^{+} \rightarrow \mathrm{N}^{+}, \mathrm{H}_{2} \mathrm{O}^{+} \rightarrow \mathrm{O}^{+}$, and $\mathrm{O}^{+}$ at $64 \mathrm{eV}$. At $362 \mathrm{eV}$ at Enceladus we have the same result except the $\mathrm{H}^{+}, \mathrm{H}_{2}^{+}$ions are missing, the $\mathrm{CO}_{2}^{+} \rightarrow \mathrm{C}^{+}$peak appears, the $\mathrm{O}_{2}^{+} \rightarrow \mathrm{O}^{+}$peak strengthens, and the $\mathrm{NH}_{3}^{+}$peak is still well resolved from the $\mathrm{O}_{2}^{+} \rightarrow \mathrm{O}^{+}$peak. Finally, at $1024 \mathrm{eV}$ for Enceladus we only see peaks due to $\mathrm{CO}_{2}^{+} \rightarrow$ $\mathrm{C}^{+}, \mathrm{O}_{2}^{+} \rightarrow \mathrm{O}^{+}, \mathrm{H}_{2} \mathrm{O}^{+} \rightarrow \mathrm{O}^{+}$, and $\mathrm{O}^{+}$. For Enceladus we do not see any obvious evidence of $\mathrm{N}^{+}$in the simulated data.

[40] Similar to what we did for the ST data we are now in a position to apply the SAM algorithm [Sittler, 1993] to our cuts through the LEF spectrograms. In Tables $5 \mathrm{~b}$ and $5 \mathrm{~d}$ we show for Dione and Enceladus, respectively, the deconvolved ion counts with error bars for the various ion species derived from the LEF simulated spectra shown in Figures $16 \mathrm{~b}$ and $17 \mathrm{~b}$. The results show that one can separate the water group ions fairly well (i.e., uncertainties $\sim 1 \%$ ) with only the $\mathrm{H}_{3} \mathrm{O}^{+}$having relatively large uncertainties $\sim 15 \%$. Well-determined ion counts for $\mathrm{H}^{+}, \mathrm{H}_{2}{ }^{+}, \mathrm{O}_{2}^{+}$, and $\mathrm{CO}_{2}^{+}$are estimated because these species are well separated from each other and the water group ions (i.e., errors $\sim 2 \%-3 \%$ ). For the LEF data we were able to include $\mathrm{NH}_{3}{ }^{+}$in our solutions whose peak is well resolved from the water group ions; estimated errors are $\sim 3-4 \%$. The data also show that we should be able to resolve the pickup ions from the ambient ions using the $\mathrm{A}$ cycle and $\mathrm{B}$ cycle data for ions $\mathrm{H}_{2}^{+}, \mathrm{NH}_{3}^{+}, \mathrm{OH}^{+}, \mathrm{H}_{2} \mathrm{O}^{+}, \mathrm{H}_{3} \mathrm{O}^{+}, \mathrm{O}_{2}^{+}, \mathrm{CO}_{2}^{+}$, and $\mathrm{CO}^{+}$.

\section{Summary and Conclusion}

[41] We have presented a model of the sputtered atmospheres for Dione and Enceladus where for simplicity we assumed spherical symmetry. We have used the maximum source rate estimated by Jurac et al. [2001a, 2001b] for both these bodies. Here the dominant sputtered species is $\mathrm{H}_{2} \mathrm{O}$. For the analysis here we assume a $10 \%$ contribution from each of the species $\mathrm{O}_{2}, \mathrm{H}_{2}, \mathrm{CO}_{2}$, and $\mathrm{NH}_{3}$. Whereas $\mathrm{O}_{2}$ and $\mathrm{H}_{2}$ are decomposition products of ice, $\mathrm{CO}_{2}$ and $\mathrm{NH}_{3}$ might be trapped in the ice matrix [e.g., Johnson et al., 2004a, 2004b]. Because these species are also more volatile than $\mathrm{H}_{2} \mathrm{O}$, their presence in the surface layers can be much less than this, which might make them difficult to detect by reflectance measurements. With these assumptions the neutral density at the surfaces of Dione and Enceladus are about a few times $10^{5} \mathrm{~mol} / \mathrm{cm}^{3}$ for $\mathrm{H}_{2} \mathrm{O}$. These densities decay roughly like $1 / \mathrm{r}^{3}$ with distance from the satellite near the body and $1 / \mathrm{r}^{2}$ far from the body. A measurement of the radial variation of the neutral density can provide information about the energy spectrum of the sputtered neutrals (i.e., determine parameter U). By measuring the height dependence of the pickup ion densities one can reconstruct the height dependence of the neutrals. We also speculated about the possibility of a gravitationally bound atmosphere of $\mathrm{O}_{3}$ and $\mathrm{O}_{2}$ because of the detection of ozone at Dione [Noll et al., 1997]. This atmosphere, if present, would be considerably denser than that estimated for a sputtered atmosphere and pickup ion densities could be dominated by $\mathrm{O}_{2}^{+}$and $\mathrm{O}_{3}^{+}$. One could readily detect the presence of Argon 40 as radioactive decay product, which would also be gravitationally bound to Dione and Enceladus, respectively.

[42] We computed the pickup ion densities as a function of height by assuming a weak interaction between the magnetosphere of Saturn and the icy satellites atmosphere. We also assumed a fluid approximation and neglected finite gyroradius effects which was substantiated by the smallness of the ion gyroradius for pickup water ions with respect to the satellite radius. In both cases water ions dominated the pickup ion density reaching peak values of $1.0 \mathrm{~mol} / \mathrm{cm}^{3}$ and $2.2 \mathrm{~mol} / \mathrm{cm}^{3}$ for Dione and Enceladus, respectively. For a wake pass at the surface of Dione in order of most abundant species, we have $\mathrm{O}_{2}^{+}, \mathrm{OH}^{+}, \mathrm{CO}_{2}^{+}, \mathrm{H}^{+}, \mathrm{H}_{3} \mathrm{O}^{+}, \mathrm{NH}_{3}^{+}, \mathrm{O}^{+}, \mathrm{H}_{2}^{+}$, and $\mathrm{NH}_{2}^{+}$. In the case of Enceladus we have in the order of most abundant at the surface $\mathrm{O}_{2}^{+}, \mathrm{H}_{3} \mathrm{O}^{+}, \mathrm{OH}^{+}, \mathrm{NH}_{3}^{+}$, and $\mathrm{CO}_{2}^{+}$. At much lower abundance we have $\mathrm{NH}_{2}^{+}, \mathrm{H}^{+}, \mathrm{O}^{+}$, and $\mathrm{H}_{2}^{+}$.

[43] We used the pickup ion densities at the surface to compute the ring distributions that would result and plotted them as a function of E/Q and angle in Saturn's equatorial plane. These simulations showed the high degree of detection for the pickup ions, even when the densities were low. This is because the pickup ions obey a delta function velocity distribution which translates into a high concentration of the ions in phase space. The flux peaks at twice the pickup velocity. We used these results to show the energyangle coverage provided by CAPS gives us ability to resolve the pickup ion distributions from the ambient plasma ions. The energy-pitch angle dependence of the 
pickup ions is considerably different from that of the ambient ions, which obey convected Maxwellians.

[44] We also computed simulated energy-TOF spectrograms for the Dione flyby and the Enceladus flyby. This data is referred to as B cycle data (see Young et al., submitted manuscript, 2003) and is accumulated over several thousand kilometers and thus provide minimal spatial resolution of the encounter. The simulations showed that the ST data could be used to separate most of the ions observed by CAPS but with considerable uncertainty for some of the minor water group ions, $\mathrm{N}^{+}$and $\mathrm{NH}_{3}^{+}$. The other ions $\mathrm{H}^{+}, \mathrm{H}_{2}^{+}, \mathrm{O}_{2}^{+}$, and $\mathrm{CO}_{2}^{+}$were well resolved by the CAPS ST data. The LEF data allowed us to resolve the water group ions, $\mathrm{N}^{+}$and $\mathrm{NH}_{3}^{+}$. In the case of $\mathrm{CO}_{2}^{+}$the LEF data was preferred since it gave a unique identification of $\mathrm{CO}_{2}^{+}$. The same can be said for $\mathrm{O}_{2}^{+}$and uniquely separating from $\mathrm{S}^{+}$. The LEF data also provides the potential for uniquely detecting $\mathrm{CO}^{+}$or $\mathrm{N}_{2}^{+}$. Therefore when we detect different ion species for high spatial, energy, and angle resolution, we will use a combination of ST and LEF data to make the ion identification. This will be done by binning the ions over specified intervals of TOF and then applying the SAM algorithm. For the B cycle data we will be able to analyze the energy-TOF spectrograms with the maximum resolution in TOF and species and to detect very minor pickup species such as $\mathrm{H}_{2}^{+}$.

[45] In conclusion, CAPS provides a unique capability in determining the composition of the surfaces of the icy satellites of Saturn [see Johnson and Sittler, 1990]. Our simulations have shown CAPS ability to detect the various ion species well above background in most cases and thus infer the bulk composition of the icy satellites. Our simulations have not included background effects. However, these effects are not expected to be important in most cases; they will primarily raise the uncertainty of our determinations. The background could be as high as $1 \%$ of the peak count rate of the dominant species at the energy it is measured. The background will tend to be flat in TOF with an increase toward smaller TOF. These corrections, which are also a function of the penetrating radiation flux, will be included under real measurement conditions. For example, if the dominant ion has a peak count of $10^{5}$ and the background is $1 \%$ or $10^{3}$ counts, then the uncertainty due to background will be $\sim 30$ counts. Such a background is primarily caused by the scattering of ions as they pass through the carbon foil of the spectrometer and their accidental detection by the start detectors. The background is made worse by penetrating radiation, as demonstrated by the Cassini Earth flyby data. Background corrections will make it more difficult to detect minor ions, such as $\mathrm{NH}_{3}^{+}$, at the few percent level relative to $\mathrm{O}^{+}$for an upstream pass while they would be detectable in a wake pass. At present only a single targeted flyby of Dione is planned. This is essentially an upstream pass with $500 \mathrm{~km}$ altitude. However, the Cassini Project is considering an extended mission for which a targeted wake pass with Dione has a high priority. In the case of $\mathrm{NH}_{3}^{+}$we must use LEF data, which has sensitivity a factor of 10 less than ST data but will be enriched relative to water group molecules because of the high electron affinity of oxygen (i.e., electrons picked up in carbon foils). We have also argued that the closer the spacecraft encounters the moon the better, in order to minimize the diluting effect of scattering of newly produced ions. A rough calculation indicates that pitch angle scattering of $5^{\circ}$ will dilute the pickup ions by a factor of 2 ; here the pickup ions are confined centrifugally by the rotation of Saturn's magnetosphere. We do plan to dither the entrance collimator above and below Saturn's equatorial plane to look for scattering effects. Our calculations are not selfconsistent since we have assumed a weak interaction. A strong strength interaction is possible at Dione, for which Pioneer 11 and Voyager 1 detected ion cyclotron waves centered on Dione's L shell. These waves are consistent with pickup ions such as the water group ions. Finally, we note that SKR radio emissions [Kurth et al., 1981; Desch and Kaiser, 1981], as observed by Voyager 1, displayed a periodicity near the orbital period of Dione which could be interpreted as being intrinsic to Dione itself. These two observational facts, combined with images of Dione displaying some geological activity [Smith et al., 1981], could indicate that the interaction between Dione and Saturn's magnetosphere is significant.

[46] Even with the above caveats, the unique capabilities of the first high-resolution, time-of-flight mass spectrometer, flown on an outer solar system mission, are expected to be spectacular. One of the added benefits of this instrument, discussed here, is its ability to give icy satellite compositional information that will supplement the spectral and imaging data from Cassini.

[47] Acknowledgments. Work was supported at Virginia by the CAPS Cassini Project and NASA's Geology and Geophysics Program. At Goddard Space Flight Center we acknowledge the support by the CAPS Cassini Project and the programming support provided by Sarabjit Bakshi (L-3 Communications EER Systems, Inc.) and Tom Vollmer (Goddard Space Flight Center, Interplanetary Physics Branch).

[48] Shadia Rifai Habbal thanks Thomas M. Orlando and John R. Spencer for their assistance in evaluating this paper.

\section{References}

Albritton, D. L. (1978), Ion-neutral reaction-rate constants measured in flow reactors through 1977, At. Nucl. Data Tables, 22, 1.

Albritton, D. L., I. Dotan, W. Lindinger, M. McFarland, J. Tellinghuisen, and F. C. Fehsenfeld (1977), Effects of ion speed distributions in flowdrift tube studies of ion-neutral reactions, J. Chem. Phys., 66, 410.

Banks, P. M., and G. Kockarts (1973), Aeronomy, Academic, San Diego, Calif.

Baragiola, R. A., R. Vidal, W. Svendsen, J. Schou, M. Shi, D. Bahr, and C. L. Atteberry (2003), Sputtering of water ice, Nucl. Instr. Methods Phys. Res., 209, 294, 303.

Barbosa, D. D. (1993), Theory and observations of electromagnetic ion cyclotron waves in Saturn's inner magnetosphere, J. Geophys. Res., 98, 9345 .

Bar-Nun, A., G. Herman, and Y. Melker (1985), Ejection of $\mathrm{H}_{2} \mathrm{O}, \mathrm{O}_{2}, \mathrm{H} 2$ and $\mathrm{H}$ from water ice by $0.5-6 \mathrm{keV} \mathrm{H}^{+}$and $\mathrm{Ne}^{+}$ion bombardment, Surface Sci., 150, 143.

Baum, W. A., T. Kreidl, J. A. Westphal, G. E. Danielson, P. K. Seidelmann, D. Pascu, and D. G. Currie (1980), Profile of Saturn's E ring, Bull. Am. Astron. Soc., 12, 700.

Baum, W. A., T. Kreidl, J. A. Westphal, G. E. Danielson, P. K. Seidelmann, D. Pascu, and D. G. Currie (1981), Saturn's E ring, Icarus, 47, 84.

Bortner, M. H., T. Baurer, and C. A. Blank (1972), Defense Nuclear Agency Reaction Rate Handbook, edited by M. H. Bortner and T. Baurer, Def. Nucl. Agency Inf. and Anal. Cent., Santa Barbara, Calif.

Brown, W. L., et al. (1982), Erosion and molecular formation in condensed gas films by electronic energy loss of fast ions, Nucl. Instrum. Methods, B1, 307 .

Carlson, R. W. (1999), A tenuous carbon dioxide atmosphere on Jupiter's moon Callisto, Science, 283, 820 .

Connerney, J. E. P., M. H. Acuna, and N. F. Ness (1983), Currents in Saturn's magnetosphere, J. Geophys. Res., 88, 8779

Desch, M. D., and M. L. Kaiser (1981), Saturn's kilometric radiation: Satellite modulation, Science, 292, 739. 
Fehsenfeld, F. C., A. L. Schmeltekopf, and E. E. Ferguson (1967), Thermalenergy ion-neutral reaction rates, VII, Some hydrogen-atom abstraction reactions, J. Chem. Phys., 46, 2802.

Fillius, W., W. H. Ip, and C. E. McIlwain (1980), Trapped radiation belts of Saturn: First look, Science, 207, 425.

Fillius, W., and C. E. McIlwain (1980), Very energetic protons in Saturn's radiation belt, J. Geophys. Res., 85, 5803.

Frank, L. A., B. G. Burek, K. L. Ackerson, J. H. Wolfe, and J. D. Mihalov (1980), Plasmas in Saturn's magnetosphere, J. Geophys. Res., 85, 5695.

Giguere, P. T., and W. F. Huebner (1978), A model of comet comae, I, Gas phase chemistry in one dimension, Astrophys. J., 223, 638.

Grundy, W. M., L. A. Young, and E. F. Young (2002), Discovery of leading-trailing asymmetry and $\mathrm{CO}_{2}$ ice on Ariel, DPS Meet. 34, Am. Astron. Soc., Washington, D. C.

Hall, D. T., P. D. Feldman, J. B. Holberg, and M. A. McGrath (1996), Florescent hydroxyl emissions from Saturn's ring atmosphere, Science, $272,516$.

Hamilton, D. P., and J. A. Burns (1994), Origin of Saturn's E ring: Self sustained, naturally, Science, 264, 550.

Hanel, R. A., et al. (1981), Infrared observations of the Saturnian system from Voyager 2, Science, 212, 192

Hanel, R. A., et al. (1982), Infrared observations of the Saturnian system from Voyager 2, Science, 215, 544.

Hartle, R. E., and C. S. Wu (1973), Effects of electrostatic instabilities on planetary and interstellar ions in the solar wind, J. Geophys. Res., 78, 5802.

Huebner, W. F., and P. T. Giguere (1980), A model of comet comae, II, Effects of solar photodissociation ionization, Astrophys. J., 238, 753.

Huebner, W. F., J. J. Keady, and S. P. Lyon (1992), Solar Photo Rates for Planetary Atmospheres and Atmospheric Pollutants, Kluwer Acad., Norwell, Mass.

Huntress, W. T., Jr. (1977), Laboratory studies of bimolecular reactions of positive ions in interstellar clouds, in comets, and in planetary atmospheres of reducing composition, Astrophys. J. Suppl. Ser., 33, 495.

Ip, W. H. (1997), On neutral cloud distributions in the Saturnian magnetosphere, Icarus, 126, 42.

Johnson, R. E. (1990), Energetic Charged-Particle Interactions With Atmospheres and Surfaces, Springer-Verlag, New York.

Johnson, R. E. (1998), Sputtering and desorption form icy surfaces, in Solar System Ices, edited by B. Schmitt and C. deBergh, pp. 303, Kluwer Acad., Norwell, Mass.

Johnson, R. E., and E. C. Sittler Jr. (1990), Sputter-produced plasma as a measure of satellite surface composition: The Cassini mission, Geophys. Res. Lett., 17, 1629.

Johnson, R. E., M. K. Pospieszalska, E. C. Sittler Jr., A. F. Cheng, L. J. Lanzerotti, and E. M. Sievka (1989), The neutral cloud and heavy ion inner torus at Saturn, Icarus, 77, 311.

Johnson, R. E., R. W. Carlson, J. F. Cooper, C. Paranicas, M. H. Moore, and M. Wong (2004a), Radiation effects on the surfaces of the Galilean Satellites, in Jupiter: Satellites, Atmosphere and Magnetosphere, edited by $\mathrm{F}$. Bagenal, in press.

Johnson, R. E., T. I. Quickenden, P. D. Cooper, A. J. McKinley, and C. Freeman (2004b), The production of oxidants in Europa's surface, Astrobio J., in press.

Jurac, S., R. E. Johnson, J. D. Richardson, and C. Paranicas (2001a), Satellite sputtering in Saturn's magnetosphere, Planet. Space Sci., 49, 319.

Jurac, S., R. E. Johnson, and J. D. Richardson (2001b), Saturn's E ring and production of the neutral torus, Icarus, 149, 384.

Jurac, S., M. A. McGrath, R. E. Johnson, J. D. Richardson, V. M. Vasyliunas, and A. Eviatar (2002), Saturn: Search for a missing water source, Geophys. Res. Lett., 29(24), 2172, doi:10.1029/2002GL015855.

Kieffer, L. J. (1969), Low-energy electron-collision cross-section data, I, Ionization, dissociation, vibrational excitation, At. Data, 1, 19

Kim, J. K., L. P. Theard, and W. T. Huntress Jr. (1975), ICR studies of some hydrogen atom abstraction reactions: $\mathrm{X}^{+}+\mathrm{H}_{2} \rightarrow \mathrm{XH}^{+}+\mathrm{H}^{*}$, J. Chem. Phys., 62, 45.

Kivelson, M. G., J. Warnecke, L. J. S. Bennett, K. K. Khurana, J. A. Linker, C. T. Russell, R. J. Walker, and C. Polanskey (1998), Ganymede's magnetosphere: Magnetometer overview, J. Geophys. Res., 103, 19,963.

Kivelson, M. G., K. K. Khurana, C. T. Russell, M. Volwerk, R. J. Walker, and C. Zimmer (2000), Galileo magnetometer measurements: A stronger case for a subsurface ocean at Europa, Science, 289, 1340.

Krimigis, S. M., and T. P. Armstrong (1982), Two-component proton spectra in the inner Saturnian magnetosphere, Geophys. Res. Lett., 9, 1143.

Krimigis, S. M., J. F. Carbary, E. P. Keath, T. P. Armstrong, L. J. Lanzerotti, and G. Gloeckler (1983), General characteristics of hot plasma and energetic particles in the Saturnian magnetosphere: Results from the Voyager spacecraft, J. Geophys. Res., 88, 8871.

Krimigis, S. M., et al. (2004), Magnetospheric Imaging Instrument (MIMI) on the Cassini mission to Saturn/Titan, Space Sci. Rev, in press.
Kurth, W. S., D. A. Gurnett, and F. L. Scarf (1981), Control of Saturn's kilometric radiation by Dione, Science, 292, 742.

Lazarus, A. J., and R. L. McNutt Jr. (1983), Low energy plasma ion observations in Saturn's magnetosphere, J. Geophys. Res., 88, 8831.

Lotz, W. (1967), Electron-impact ionization cross-sections and ionization rate coefficients for atoms and ions, Astrophys. J. Suppl. Ser., 14, 207.

Lutz, B. L., C. de Bergh, and T. Owen (1983), Titan: Discovery of carbon monoxide in its atmosphere, Science, 220, 1374.

Ma, T. Z., D. A. Gurnett, and C. K. Goertz (1987), Interpretation of electrostatic noise observed by Voyager 1 in Titan's wake, J. Geophys. Res., 92, 8595

Madey, T. E., R. E. Johnson, and T. M. Orlando (2002), Far-out surface science: radiation-induced surface processes in the solar system, Surface Sci., 500, 838

Mark, T. D., F. Egger, and M. Cheret (1977), Ionization of ammonia and deuterated ammonia by electron impact from threshold up to $180 \mathrm{eV}$, J. Chem. Phys., 67, 3795.

Massey, H. S. W., and H. B. Gilbody (1974), Electronic and ionic impact phenomena, in Recombination and Fast Collisions of Heavy Particles, vol. IV, 2782 pp., Oxford Univ. Press, New York.

Maurice, S., E. C. Sittler Jr., J. F. Cooper, B. H. Mauk, M. Blanc, and R. S. Selesnick (1996), Comprehensive analysis of electron observations at Saturn: Voyager 1 and 2, J. Geophys. Res., 101, 15,211.

McComas, D. J., and J. E. Nordholt (1990), A new approach to 3-D, high sensitivity, high mass resolution space plasma composition measurements, Rev. Sci. Instrum., 61, 3095.

McComas, D. J., J. E. Nordholt, J.-J. Berthelier, J.-M. Illiano, and D. T. Young (1998), The Cassini Ion Mass Spectrometer, in Measurement Techniques in Space Plasmas: Particles, Geophys. Monogr. Ser, vol. 102 , edited by R. F. Pfaff, J. E. Borovsky, and D. T. Young, p. 187, AGU, Washington, D. C.

McCord, T. B., et al. (1998), Organics and other molecules in the surfaces of Ganymede and Callisto, Science, 280, 1242.

Morrison, D., T. V. Johnson, E. M. Shoemaker, L. A. Soderblom, P. Thomas, J. Veverka, and B. A. Smith (1984), Satellites of Saturn: Geological perspective, in Saturn, edited by T. Gehrels and M. S. Matthews, Univ. of Ariz. Press, Tucson.

Neubauer, F. M., D. A. Gurnett, J. D. Scudder, and R. E. Hartle (1984), Titan's magnetospheric interaction, in Saturn, edited by T. Gehrels and M. S. Matthews, p. 760, Univ. of Ariz. Press, Tucson.

Newman, J. H., J. D. Cogan, D. L. Ziegler, D. E. Nitz, R. D. Rundel, K. A. Smith, and R. F. Stebbilngs (1982), Charge transfer in $\mathrm{H}^{+}-\mathrm{H}$ and $\mathrm{H}^{+}-\mathrm{D}$ collisions within the energy range $0.1-150 \mathrm{eV}$, Phys. Rev. A, 25, 2976.

Noll, K. S., T. Rousch, D. Cruikshank, and R. E. Johnson (1997), Detection of ozone on Saturn's satellites Dione and Rhea, Nature, 388, 45.

Nordholt, J., E. J.-J. Berthelier, D. M. Burr, H. O. Funsten, R. Goldstein, J.-M. Illiano, K. P. McCabe, D. J. McComas, D. M. Potter, and D. T. Young (1998), The Cassini Ion Mass Spectrometer: Performance metrics and techniques, in Measurement Techniques in Space Plasmas: Particles, Geophys. Monogr. Ser., vol. 102, edited by R. F. Pfaff, J. E. Borovsky, and D. T. Young, p. 209, AGU, Washington, D. C.

Oppenheimer, M. (1975), Gas phase chemistry in comets, Astrophys. J., $196,251$.

Orient, O. J., and S. K. Srivastava (1987), Electron impact ionization of $\mathrm{H}_{2} \mathrm{O}, \mathrm{CO}, \mathrm{CO}_{2}$ and $\mathrm{CH}_{4}$, J. Phys. B At. Mol. Phys., 20, 3923.

Paranicas, C., A. F. Cheng, B. H. Mauk, E. F. Keath, and S. M. Krimigis (1997), Evidence of a source of energetic ions at Saturn, J. Geophys. Res., 104, 17,459.

Richardson, J. D. (1986), Thermal ions at Saturn: Plasma parameters and implications, J. Geophys. Res., 91, 1381.

Richardson, J. D., and E. C. Sittler Jr. (1990), A plasma density model for Saturn based on Voyager observations, J. Geophys. Res., 95, 12,019.

Richardson, J. D., A. Eviatar, and G. L. Siscoe (1986), Satellite Tori at Saturn, J. Geophys. Res., 91, 8749.

Richardson, J. D., A. Eviatar, M. A. McGrath, and V. M. Vasyliunas (1998), $\mathrm{OH}$ in Saturn's magnetosphere: Observations and implications, J. Geophys. Res., 103, 20,245.

Rudd, M. E., Y.-K. Kim , D. H. Madison, and J. W. Gallagher (1985), Electron production in proton collisions: total cross sections, Rev. Mod. Phys. 57, 965.

Russell, C. T., and D. E. Huddleston (2000), Ion-cyclotron waves at Io, Planet. Ionospheres Magnetospheres, 26, 1505.

Russell, C. T., D. E. Huddleston, K. K. Khurana, and M. G. Kivelson (2000), Waves and fluctuations in the jovian magnetosphere, Planet. Ionospheres Magnetospheres, 26, 1489.

Samuelson, R. E., W. C. Maguire, R. A. Hanel, V. G. Kunde, D. E. Jennings, Y. L. Yung, and A. C. Aikin (1983), $\mathrm{CO}_{2}$ on Titan, J. Geophys. Res., 88, 8709. 
Schardt, A. W., and F. B. McDonald (1983), The flux and source of energetic protons in Saturn's inner magnetosphere, J. Geophys. Res. $88,8923$.

Schardt, A. W., K. W. Behannon, R. P. Lepping, J. F. Carbary, A. Eviatar, and G. L. Siscoe (1984), The outer magnetosphere, in Saturn, edited by T. Gehrels and M. S. Matthews, Univ. of Ariz. Press, Tucson.

Shemansky, D. E., P. Matherson, D. T. Hall, H. Y. Hu, and T. M. Tripp (1993), Detection of the hydroxyl radical in the Saturn magnetosphere, Nature, 363, 329.

Sieger, M. T., W. C. Simpson, and T. M. Orlando (1998), Production of $\mathrm{O}_{2}$ on icy satellites by electronic excitation of low-temperature water ice, Nature, 394, 554.

Sieveka, E. M. (1983), Charged particle erosion of icy satellites: Coronal atmospheres and surface redistribution, Ph.D. thesis, Univ. of Virginia, Charlottesville, Va.

Sittler, E. C., Jr. (1993), Real-time spectral analysis algorithm for space plasma three-dimension ion mass spectrometers, Rev. Sci. Instrum., 64, 2771.

Sittler, E. C., Jr., K. W. Ogilvie, and J. D. Scudder (1983), Survey of low energy plasma electrons in Saturn's magnetosphere: Voyager 1 and 2, J. Geophys. Res., 88, 8847.

Smith, E. J., and B. T. Tsurutani (1983), Saturn's magnetosphere: Observations of ion cyclotron waves near the Dione L shell, J. Geophys. Res., 88, 7831.

Smith, E. J., et al. (1981), Encounter with Saturn: Voyager 1 imaging science results, Science, 212, 163.

Spencer, J. (1998), Upper limits for condensed $\mathrm{O}_{2}$ on Saturn's icy satellites and rings, Icarus, 136, 349.

Stebbings, R. F., and J. A. Rutherford (1968), Low-energy collisions between $\mathrm{O}^{+}\left({ }^{4} \mathrm{~S}\right)$ and $\mathrm{H}\left({ }^{1} \mathrm{~S}\right), J$. Geophys. Res., 73, 1035.

Stebbings, R. F., A. C. H. Smith, and H. Ehrhardt (1964), Charge transfer between oxygen atoms and $\mathrm{O}^{+}$and $\mathrm{H}^{+}$ions, J. Geophys. Res., 69, 2349. Stevenson, D. J. (1982), Volcanism and igneous processes in small icy satellites, Nature, 298, 142.

Tawara, H. (1978), Cross sections for charge transfer of hydrogen beams in gases and vapors in the energy range $10 \mathrm{eV}-10 \mathrm{keV}$, At. Data Nucl. Data Tables, 22, 491.

Van Allen, J. A. (1982), Findings on rings and inner satellites of Saturn by Pioneer 11, Icarus, 51, 509.

Van Allen, J. A. (1984), Energetic particles in the inner magnetosphere of Saturn, in Saturn, edited by T. Gehrels and M. S. Matthews, Univ. of Ariz. Press, Tucson.
Van Allen, J. A., M. F. Thomsen, B. A. Randall, R. L. Rairden, and C. L. Grosskreutz (1980a), Saturn's magnetosphere, rings, and inner satellites, Science, 207, 415

Van Allen, J. A., B. A. Randall, and M. F. Thomsen (1980b), Sources and sinks of energetic electrons and protons in Saturn's magnetosphere, J. Geophys. Res., 85, 5679.

Vasyliunas, V. M., and G. L. Siscoe (1976), On the flux and the energy spectrum of interstellar ions in the solar system, J. Geophys. Res., 81, 1247. Vogt, R. E., D. L. Chenette, A. C. Cummings, T. L. Garrard, E. C. Stone, A. W. Schardt, J. H. Trainor, N. Lal, and F. B. McDonald (1981), Energetic charged particles in Saturn's magnetosphere: Voyager 1 results, Science, 212, 231.

Vogt, R. E., D. L. Chenette, A. C. Cummings, T. L. Garrard, E. C. Stone, A. W. Schardt, J. H. Trainor, N. Lal, and F. B. McDonald (1982), Energetic charged particles in Saturn's magnetosphere: Voyager 2 results, Science, 215, 577 .

Watson, C. C. (1982), The sputter-generated planetary coronae: Galilean satellites of Jupiter, Proc. Lunar Planet. Sci. Conf., 12th, 1560.

Wu, C. S., and R. C. Davidson (1972), Electromagnetic instabilities produced by neutral-particle ionization in interplanetary space, J. Geophys. Res., 77, 5399.

Zimmer, C., K. K. Khurana, and M. G. Kivelson (2000), Subsurface oceans on Europa and Callisto: Constraints from Galileo magnetometer observations, Icarus, 147, 329

F. Crary and D. T. Young, Division of Space Science and Engineering, Southwest Research Institute, 6220 Culebra Road, P. O. Drawer 28510, San Antonio, TX 78228-0510, USA. (fcrary@swri.edu; dyoung@swri.edu)

R. E. Johnson, Engineering Physics, University of Virginia, Thornton Hall, Charlottesville, VA 22904, USA. (rej@virgini.edu)

S. Jurac and J. D. Richardson, Center for Space Research, Massachusetts Institute of Technology, Rm. 37-635, Cambridge, MA 02139, USA. (jurac@ space.mit.edu; jdr@space.mit.edu)

M. McGrath, Space Telescope Science Institute, 3700 San Martin Drive, Baltimore, MD 21218, USA. (mcgrath@stsci.edu)

J. E. Nordholt, Space and Atmospheric Science Group, Los Alamos National Laboratory, Mail Stop P-23, M/S H803, Los Alamos, NM 87545, USA. (jnordholt@lanl.gov)

E. C. Sittler, NASA Goddard Space Flight Center, Code 692, Greenbelt Road, Greenbelt, MD 20771, USA. (edward.c.sittler@nasa.gov) 